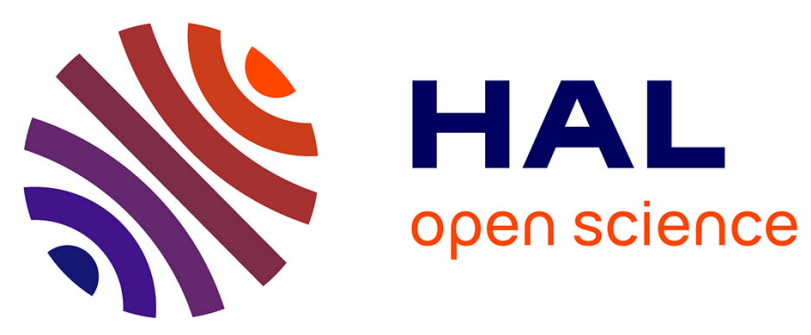

\title{
Displaying Updates in Logic
}

Guillaume Aucher

\section{To cite this version:}

Guillaume Aucher. Displaying Updates in Logic. Journal of Logic and Computation, 2016, 26 (6), pp.1865-1912. 10.1093/logcom/exw001 . hal-01476234v2

\section{HAL Id: hal-01476234 \\ https://hal.inria.fr/hal-01476234v2}

Submitted on 15 Jul 2020 (v2), last revised 27 Feb 2023 (v3)

HAL is a multi-disciplinary open access archive for the deposit and dissemination of scientific research documents, whether they are published or not. The documents may come from teaching and research institutions in France or abroad, or from public or private research centers.
L'archive ouverte pluridisciplinaire HAL, est destinée au dépôt et à la diffusion de documents scientifiques de niveau recherche, publiés ou non, émanant des établissements d'enseignement et de recherche français ou étrangers, des laboratoires publics ou privés. 


\title{
Displaying Updates in Logic*
}

\author{
Guillaume Aucher \\ University of Rennes 1 \\ INRIA \\ 263, Avenue du Général Leclerc \\ 35042 Rennes Cedex, France \\ email: guillaume.aucher@irisa.fr
}

July 15,2020

\begin{abstract}
We generalize the language of substructural logics interpreted over the ternary relational semantics and introduce a logic called update logic. This is motivated by our intention to capture within the logical framework of substructural logics various logic-based formalisms dealing with common sense reasoning and logical dynamics. This initiative is based on the key observation that an update can be represented abstractly by the ternary relation of the substructural framework. Thus, we introduce three triples of connectives which are interconnected by means of cyclic permutations. The usual fusion, implication and coimplication connectives form one of these triples. We define a proper display calculus for our language which generalizes the display calculus for modal logic. We do not resort to structural connectives for truth constants and we show how we can obtain our display rules using Gaggle Theory. We prove the soundness and strong completeness of our display calculus via a Henkin-style construction. Using correspondence results from substructural logics, we also obtain sound and complete display calculi for a wide variety of classical and substructural logics. Then, we define dual substructural connectives and we provide a display calculus for our language extended with these dual connectives. Finally, we focus on the specific case of intuitionistic logic for which we provide a sound and strongly complete display calculus. In a companion article [7], we provide a sequent calculus for update logic which generalizes the non-associative Lambek calculus and a sequent calculus for dynamic epistemic logic which extends this sequent calculus for update logic.
\end{abstract}

${ }^{*}$ This paper corrects some errors in the article with the same title published in the Journal of Logic and Computation, Volume 26, Number 6 on March 2, 2016 (doi:10.1093/logcom/exw001). The corrections concern dual update logic. There was a typographical mistake in the truth conditions of the dual connectives in Definition 20: "y" and "z" should be swapped. The inference rules of the corresponding proof system in Figure 11 had also to be corrected. The second related set of corrections concerns the case study of Section 8. It now only deals with intuitionistic logic and not with $b i$-intuitionistic logic anymore. The results related to that dual part were erroneous. 


\section{Introduction}

In everyday life the way we update and revise our beliefs plays an important role in our representation of the surrounding world and therefore also in our decision making process. This has lead researchers in artificial intelligence and computer science to develop logic-based theories that study and formalize belief change and the so-called "common sense reasoning". The rationale underlying the development of such theories is that it would ultimately help us understand our everyday life reasoning and the way we update our beliefs, and that the resulting work could subsequently lead to the development of tools that could be used, for example, by artificial agents in order to act autonomously in an uncertain and changing world. A number of theories have been proposed to capture different kinds of updates and the reasoning styles that they induce, using different formalisms and under various assumptions: dynamic epistemic logic [73, 74], default and non-monotonic logics [45, 28], belief revision theory [29], conditional logic [53], etc. However, a generic and general framework encompassing all these theories is still lacking. Instead, the current state of the art is such that we are left with various formalisms which are difficult to relate formally to each other despite numerous attempts [30, 46, 4, 75, 3, 13, 32, 20], partly because they rely on different kinds of formalisms. This is problematic if logic is to be viewed ultimately as a unified and unifying field and if we want to avoid that logic goes on "riding off madly in all directions" (a metaphor used by van Benthem [73]).

We propose that the very general framework of substructural logics be considered as the logical framework unifying and capturing all these logics. This proposal is based on the key observation that an update can be represented abstractly as a ternary relation: the first argument of the ternary relation represents the initial situation/state, the second argument represents the event that occurs in this initial situation (the informative input) and the third argument represents the resulting situation/state after the occurrence of the event. Indeed, the semantics of substructural logics also relies on a ternary relation introduced by Routley and Meyer [63, 64, 65, 66] for relevance logic in the 1970's. Nevertheless, providing a non-circular and conceptually grounded interpretation of this ternary relation remains problematic [16]. As we shall see, our dynamic interpretation of ternary relations as updates provides a conceptual foundation for the Routley and Meyer semantics which is also consistent with some of the interpretations of this ternary relation proposed in the literature.

In this article, as a preliminary step towards achieving our objective of unifying the logics of common sense reasoning, we will generalize the substructural language. We believe that this will allow us to account appropriately for the fine-grained reasoning present in common sense reasoning (such as for example default, abductive, inductive, retractive or preemptive reasoning). As we will see, this generalization will already capture not only a very wide range of substructural and non-classical logics, but also their dual versions. We will also provide display calculi for all these substructural and non-classical logics. Display calculi are extensions of Gentzen calculi to deal with non-classical logics. Gentzen calculi [31] can be viewed as attempts to model as adequately as possible the classical reasoning of mathematicians. Hence, display calculi can somehow be viewed as attempts to model the non-classical reasoning of humans. So, given our overall objective, it makes perfect sense to develop and study display calculi for substructural and non-classical logics insofar as substructural and non-classical logics are apt to 
capture common sense reasoning.

Our contributions in the article are organized as follows. We will start by briefly recalling elementary notions of relevance and substructural logics and we will observe that the ternary relation of relevance logic [61, 62, 25] can be interpreted intuitively as a kind of update (Section 2). Then, after some motivating examples, we will introduce update logic (Section 3). Update logic generalizes the usual language of substructural logics interpreted over the relational semantics. We introduce three symmetric triples of connectives which are semantically interconnected by means of cyclic permutations (see explanations after Definition 8). One of these triples consists of the usual fusion, implication and co-implication connectives. The logical language we obtain is therefore more expressive than the usual language of substructural logics. Then, we will define a proper display calculus for our language which generalizes the display calculus for modal logic (Section 4). We will show how to obtain the display rules of this display calculus using Dunn's Gaggle Theory [24]. However, we will not resort to structural connectives for truth constants. These structural constants can in fact be reintroduced as structural abbreviations. We will prove the soundness and strong completeness of our display calculus using a Henkin-style construction (Section 5). Using correspondence results from substructural logics, we will also obtain sound and complete display calculi for a wide variety of classical and substructural logics by adding appropriate structural rules (Section 6). Then, we will define dual substructural connectives and we will provide a display calculus for our language extended with these dual connectives (Section 7). We will then focus on the specific case of intuitionistic logic for which we will provide a sound and strongly complete display calculus (Section 8). Finally, we will conclude (Section 9).

Remark 1. For the reader mainly interested in technical results, all these results are summarized in Sections 7 and 8 (except for Proposition 1): Theorems 18, 19 and 23 (for soundness and completeness), Theorem 17 (for cut admissibility), Propositions 1 and 16 (for expressiveness) and Corollary 2 (for conservativity). All the other results in this article are specific instances of these general results.

\section{Updates as a Conceptual Foundation for Substructural Logics}

Substructural logics are a family of logics lacking some of the structural rules of classical logic. A structural rule is a rule of inference which is closed under substitution of formulas [61, Definition 2.23]. In a certain sense, a structural rule allows to manipulate the structure(s) of the sequent/consecution without altering its logical content. The structural rules for classical logic introduced by Gentzen [31] are given in Figure 11. The comma in these sequents has to be interpreted as a conjunction in an antecedent and as a disjunction in a consequent. While Weakening $\left(W_{A}, W_{K}\right)$ and Contraction $\left(C_{A}, C_{K}\right)$ are often dropped as in relevance logic and linear logic, the rule of Permutation $\left(P_{A}, P_{K}\right)$ is often preserved. When some of these rules are dropped, the comma ceases to behave as a conjunction (in the antecedent) or a disjunction (in the consequent). In that case the comma corresponds to other substructural connectives and we often introduce new punctuation marks which do not fulfill all these structural rules to deal with these new substructural connectives. 
Weakening:

$$
\begin{aligned}
& \frac{U \vdash V}{X, U \vdash V} W_{A} \\
& \frac{U \vdash V}{U \vdash V, X} W_{K}
\end{aligned}
$$

\section{Contraction:}

$$
\frac{X, X, U \vdash V}{X, U \vdash V} C_{A}
$$$$
\frac{U \vdash V, X, X}{U \vdash V, X} C_{K}
$$

\section{Permutation:}

$\frac{U, Y, X, V \vdash W}{U, X, Y, V \vdash W} P_{A}$

$\frac{U \vdash V, Y, X, W}{U \vdash V, X, Y, W} P_{K}$

Figure 1: Gentzen's Structural Rules for Classical Logic

\subsection{Substructural Logics}

Our exposition of substructural logics is based on [61, 62, 25] (see also [54] for a general introduction) 1 The logical framework presented by Restall [61] is more general and deals with a wide range of substructural logics: relevant logic, linear logic, Lambek calculus, arrow logic, etc. We will only introduce a fragment of this general framework in order to highlight the main new ideas. In particular, we will not consider truth sets and we will assume that our logics do not reject distribution. These other features can be added and our framework can be adapted, following the exposition of Restall [61].

The semantics of substructural logics is based on the ternary relation of the frame semantics for relevant logic originally introduced by Routley and Meyer [63, 64, 65, 66]. Another semantics proposed independently by Urquhart [67, 68, 69] at about the same time will be discussed at the end of this section.

In the sequel, $\mathbb{P}$ is a countable set of propositional letters and we consider the following set of logical connectives:

$$
\text { Sub : }=\left\{\top, \perp, \square, \diamond^{-}, \neg, \vee, \wedge, \otimes, \supset, \subset, \Rightarrow\right\}
$$

We also define the set of connectives Sub $:=\mathrm{Sub}-\{\Rightarrow\}$ (the connective $\Rightarrow$ corresponds to the intuitionistic implication).

Definition 1 (Languages $\mathcal{L}\left(\mathbb{P}\right.$, Sub) and $\mathcal{L}\left(\mathbb{P}\right.$, Sub$\left._{-}\right)$). The language $\mathcal{L}(\mathbb{P}$, Sub $)$ is the language associated to $S u b$ and $\mathbb{P}$, that is, the language built compositionally from the connectives of Sub and the set of propositional letters $\mathbb{P}$. More formally, it is the set of formulas defined inductively by the following grammar in Backus-Naur Form (BNF), where $p$ ranges over $\mathbb{P}$ :

$$
\mathcal{L}(\mathbb{P}, \text { Sub }): \quad \varphi \quad:=\begin{array}{c|c|c|c|c|c|}
\top & \perp & p & (\varphi \wedge \varphi) & (\varphi \vee \varphi) & \mid
\end{array}|(\varphi \Rightarrow \varphi)|
$$

The language $\mathcal{L}\left(\mathbb{P}\right.$, Sub$\left._{-}\right)$is the language $\mathcal{L}(\mathbb{P}$, Sub) without the (intuitionistic) connective $\Rightarrow$.

\footnotetext{
${ }^{1}$ We very slightly change the definitions of frames and models as they are defined in [61] (we give the details of these differences in the sequel). The definitions remain equivalent nevertheless.
} 
Definition 2 (Point set, accessibility relation). A point set $\mathcal{P}=(P, \sqsubseteq)$ is a non-empty set $P$ together with a partial order $\sqsubseteq$ on $P$. The set $\operatorname{Prop}(\mathcal{P})$ of propositions on $\mathcal{P}$ is the set of all subsets $X$ of $P$ which are closed upwards: that is, if $x \in X$ and $x \sqsubseteq x^{\prime}$ then $x^{\prime} \in X$. When $\sqsubseteq$ is the identity relation $=$, we say that $\mathcal{P}$ is flat. We abusively write $x \in \mathcal{P}$ for $x \in P$.

- A binary relation $R$ is a positive two-place accessibility relation on the point set $\mathcal{P}$ if, and only if, for any $x, y \in \mathcal{P}$ where $x R y$, if $x^{\prime} \sqsubseteq x$ then there is a $y^{\prime} \sqsupseteq y$ such that $x^{\prime} R y^{\prime}$. Similarly, if $x R y$ and $y \sqsubseteq y^{\prime}$ then there is some $x^{\prime} \sqsubseteq x$ such that $x^{\prime} R y^{\prime}$.

- A binary relation $R$ is a plump positive two-place accessibility relation on the point set $\mathcal{P}$ if, and only if, for any $x, y, x^{\prime}, y^{\prime} \in \mathcal{P}$, where $x R y, x^{\prime} \sqsubseteq x$ and $y \sqsubseteq y^{\prime}$ it follows that $x^{\prime} R y^{\prime}$.

- A ternary relation $\mathcal{R}$ is a three-place accessibility relation on the point set $\mathcal{P}$ if, and only if, whenever $\mathcal{R} x y z$ and $z \sqsubseteq z^{\prime}$ then there are $y^{\prime} \sqsupseteq y$ and $x^{\prime} \sqsupseteq x$ such that $\mathcal{R} x^{\prime} y^{\prime} z^{\prime}$. Similarly, if $x^{\prime} \sqsubseteq x$ then there are $y^{\prime} \sqsubseteq y$ and $z^{\prime} \sqsupseteq z$ such that $\mathcal{R} x^{\prime} y^{\prime} z^{\prime}$, and if $y^{\prime} \sqsubseteq y$ then there are $x^{\prime} \sqsubseteq x$ and $z^{\prime} \sqsupseteq z$, such that $\mathcal{R} x^{\prime} y^{\prime} z^{\prime}$.

- A ternary relation $\mathcal{R}$ is a plump three-place accessibility relation on the point set $\mathcal{P}$ if, and only if, for any $x, y, z, x^{\prime}, y^{\prime}, z^{\prime} \in \mathcal{P}$ such that $\mathcal{R} x y z$, if $x^{\prime} \sqsubseteq x, y^{\prime} \sqsubseteq y$ and $z \sqsubseteq z^{\prime}$, then $\mathcal{R} x^{\prime} y^{\prime} z^{\prime}$.

We say that $Q$ is an accessibility relation if, and only if, it is either a positive two-place or a three-place accessibility relation.

Note that plump accessibility relations are accessibility relations. The definitions of accessibility relations relate $R, \mathcal{R}$ with $\sqsubseteq$. They are set in such a way that condition (Persistence) can be lifted to arbitrary formulas of $\mathcal{L}(\mathbb{P}$, Sub $)$ and holds not only for the propositional letters of $\mathbb{P}$.

Definition 3 (Substructural model). A substructural model is a tuple $\mathcal{M}=(\mathcal{P}, R, \mathcal{R}, \mathcal{I})$ where:

- $\mathcal{P}=(P, \sqsubseteq)$ is a point set;

- $R \subseteq \mathcal{P} \times \mathcal{P}$ is a (binary) accessibility relation on $\mathcal{P}$;

- $\mathcal{R} \subseteq \mathcal{P} \times \mathcal{P} \times \mathcal{P}$ is a (ternary) accessibility relation on $\mathcal{P}$;

- $\mathcal{I}: P \rightarrow 2^{\mathbb{P}}$ is a function called the interpretation function satisfying moreover the condition $\{x \in \mathcal{M} \mid p \in \mathcal{I}(x)\} \in \operatorname{Prop}(\mathcal{P})$, which can be reformulated as follows: for all $x, y \in P$ and all $p \in \mathbb{P}$,

$$
\text { if } p \in \mathcal{I}(x) \text { and } x \sqsubseteq y \text { then } p \in \mathcal{I}(y) .
$$

(Persistence)

We abusively write $x \in \mathcal{M}$ for $x \in \mathcal{P}$ and $(\mathcal{M}, x)$ is called a pointed substructural model. The class of all pointed substructural models is denoted $\mathcal{E}$. A (pointed) substructural frame is a (pointed) substructural model without interpretation function. The class of all pointed substructural frames is denoted $\mathcal{F}$. 
Definition 4 (Evaluation relation). Let $\mathcal{M}$ be a substructural model, $x \in \mathcal{M}$ and $\varphi \in \mathcal{L}(\mathbb{P}$, Sub). The evaluation relation $\mathcal{M}, x \Vdash \varphi$ is defined inductively as follows:

$$
\begin{aligned}
& \mathcal{M}, x \| \top \quad \text { always; } \\
& \mathcal{M}, x \Vdash \perp \quad \text { never; } \\
& \mathcal{M}, x \| p \quad \text { iff } \quad p \in \mathcal{I}(x) \text {; } \\
& \mathcal{M}, x \Vdash \varphi \wedge \psi \quad \text { iff } \quad \mathcal{M}, x \| \varphi \text { and } \mathcal{M}, x \Vdash \psi ; \\
& \mathcal{M}, x \Vdash \varphi \vee \psi \quad \text { iff } \quad \mathcal{M}, x \| \varphi \text { or } \mathcal{M}, x \| \psi \text {; } \\
& \mathcal{M}, x \| \square \varphi \quad \text { iff } \quad \text { for all } y \in \mathcal{P} \text {, such that } x R y, \mathcal{M}, y \| \varphi \text {; } \\
& \mathcal{M}, x \| \diamond^{-} \varphi \quad \text { iff } \quad \text { there is } y \in \mathcal{P} \text { such that } y R x \text { and } \mathcal{M}, y \| \varphi \text {; } \\
& \mathcal{M}, x \| \varphi \otimes \psi \quad \text { iff } \quad \text { there are } y, z \in \mathcal{P} \text { such that } \mathcal{R} y z x \text {, } \\
& \mathcal{M}, y \Vdash \varphi \text { and } \mathcal{M}, z \| \psi ; \\
& \mathcal{M}, x \| \varphi \supset \psi \quad \text { iff } \quad \text { for all } y, z \in \mathcal{P} \text { such that } \mathcal{R} x y z \\
& \text { if } \mathcal{M}, y \| \varphi \text { then } \mathcal{M}, z \Vdash \psi \text {; } \\
& \mathcal{M}, x \| \psi \subset \varphi \quad \text { iff } \quad \text { for all } y, z \in \mathcal{P} \text { such that } \mathcal{R} y x z, \\
& \text { if } \mathcal{M}, y \| \varphi \text { then } \mathcal{M}, z \Vdash \psi \text {; } \\
& \mathcal{M}, x \Vdash \varphi \Rightarrow \psi \quad \text { iff } \quad \text { for all } y \in P \text {, if } x \sqsubseteq y \text { then not } \mathcal{M}, y \Vdash \varphi \text { or } \mathcal{M}, y \Vdash \psi \text {. }
\end{aligned}
$$

We extend these definitions to the class of pointed substructural frames. We define the evaluation relation $\Vdash \subseteq \mathcal{F} \times \mathcal{L}(\mathbb{P}$, Sub $)$ as follows. Let $(F, x)$ be a pointed frame and let $\varphi \in \mathcal{L}(\mathbb{P}$, Sub $)$. Then, we have that

$F, x \Vdash \varphi \quad$ iff $\quad$ for all interpretation functions $\mathcal{I}$ such that $(F, \mathcal{I})$ satisfies Persistence. $(F, \mathcal{I}), x \Vdash \varphi$

A substructural model stripped out from its interpretation function corresponds to a frame as defined in [61, Definition 11.8] and without truth sets. In [61], a model is a frame together with an evaluation relation.

Urquhart's semantics. The Urquhart's semantics for relevance logic was developed independently from the Routley-Meyer's semantics in the early 1970's. An operational frame is a set of points $\mathcal{P}$ together with a function which gives us a new point from a pair of points:

$$
\sqcup: \mathcal{P} \times \mathcal{P} \rightarrow \mathcal{P} .
$$

An operational model is then an operational frame together with a relation $\Vdash$ which indicates what formulas are true at what points. The truth conditions for the implication $\supset$ are defined as follows:

$$
x \Vdash \varphi \supset \psi \text { iff for each } y \text {, if } y \Vdash \varphi \text { then } x \sqcup y \Vdash \psi
$$

As one can easily notice, an operational frame is a Routley-Meyer frame where $\mathcal{R} x y z$ holds if and only if $x \sqcup y=z$. Hence, the ternary relation $\mathcal{R}$ of the Routley-Meyer semantics is a generalization of the function $\sqcup$ of the Urquhart's semantics. Because it is a relation, it allows moreover to apply $x$ to $y$ and yield either a set of outcomes or no outcome at all. 


\subsection{Updates as Ternary Relations}

The ternary relation of the Routley and Meyer semantics was introduced originally for technical reasons: any 2-ary ( $n$-ary) connective of a logical language can be given a semantics by resorting to a 3-ary (resp. $n+1$-ary) relation on worlds. In fact, this may be the most general and abstract way of providing a semantics for two-ary conditionals of the form $\varphi \supset \psi$. Subsequently, a number of philosophical interpretations of this ternary relation have been proposed and we will briefly recall some of them at the end of this section (see [16, 62, 48] for more details). However, one has to admit that providing a non-circular and conceptually grounded interpretation of this relation remains problematic [16]. In this article we propose a new dynamic interpretation.

Our proposal is based on the key observation that an update can be represented abstractly as a ternary relation: the first argument of the ternary relation represents the initial situation/state, the second the event that occurs in this initial situation (the informative input) and the third the resulting situation/state after the occurrence of the event. With this interpretation in mind, $\mathcal{R} x y z$ reads as 'the occurrence of event $y$ in world $x$ results in the world $z$ ' and the corresponding conditional $\chi \supset \varphi$ reads as 'the occurrence in the current world of an event satisfying property $\chi$ results in a world satisfying $\varphi$ '.

This interpretation is coherent with a number of interpretations of the ternary relation proposed in substructural logic. In substructural logics, points are sometimes also called worlds, states, situations, set-ups, and as explained by Restall:

"We have a class of points (over which $x$ and $y$ vary), and a function $\sqcup$ which gives us new points from old. The point $x \sqcup y$ is supposed, on Urquhart's interpretation, to be the body of information given by combining $x$ with $y$." [62, p. 363]

and also, keeping in mind the truth conditions for the connective $\supset$ of Expression (2):

"To be committed to $A \supset B$ is to be committed to $B$ whenever we gain the information that $A$. To put it another way, a body of information warrants $A \supset B$ if and only if whenever you update that information with new information which warrants $A$, the resulting (perhaps new) body of information warrants $B . "$ (emphasis added) [62, p. 362]

Moreover, as explained by Restall, this substructural "update" can be nonmonotonic and may correspond to some sort of revision:

"[C]ombination is sometimes nonmonotonic in a natural sense. Sometimes when a body of information is combined with another body of information, some of the original body of information might be lost. This is simplest to see in the case motivating the failure of $A \vdash B \supset A$. A body of information might tell us that $A$. However, when we combine it with something which tells us $B$, the resulting body of information might no longer warrant $A$ (as $A$ might with $B$ ). Combination might not simply result in the addition of information. It may well warrant its revision." (my emphasis) [62, p. 363] 
Our dynamic interpretation of the ternary relation is consistent with the above considerations: sometimes updating beliefs amounts to revise beliefs.

The dynamic reading of the ternary relation and its corresponding conditional is very much in line with the so-called "Ramsey Test" of conditional logic. The Ramsey test can be viewed as the very first modern contribution to the logical study of conditionals and much of the contemporary work on conditional logic can be traced back to the famous footnote of Ramsey [58] $]^{2}$ Roughly, it consists in defining a counterfactual conditional in terms of belief revision: an agent currently believes that $\varphi$ would be true if $\psi$ were true $($ i.e. $\psi \supset \varphi)$ if and only if he should believe $\varphi$ after learning $\psi$. A first attempt to provide truth conditions for conditionals, based on Ramsey's ideas, was proposed by Stalnaker. He defined his semantics by means of selection functions over possible worlds $f: W \times 2^{W} \rightarrow W$. As one can easily notice, Stalnaker's selection functions could also be considered from a formal point of view as a special kind of ternary relation, since a relation $\mathcal{R}_{f} \subseteq W \times 2^{W} \times W$ can be canonically associated to each selection function $f !^{3}$ So, the dynamic reading of the ternary semantics is consistent with the dynamic reading of conditionals proposed by Ramsey.

This dynamic reading was not really considered or investigated by substructural logicians when they connected the substructural ternary semantics with conditional logic [16]. On the other hand, the dynamic reading of inferences has been stressed to a large extent by van Benthem [71, 73] and also by Baltag \& Smets [11, 12, 13]. Our dynamic interpretation of the ternary semantics of substructural logics is consistent with the interpretations proposed by substructural logicians. In fact, our point of view is also very much in line with the claim of Gärdenfors and Makinson [30, 46] that non-monotonic reasoning and belief revision are "two sides of the same coin": as a matter of fact, non-monotonic reasoning is a reasoning style and belief revision is a sort of update. The formal connection in this case also relies on a similar idea based on the Ramsey test.

To summarize our discussion, our dynamic interpretation of the ternary relation of substructural logic is intuitive and consistent, in the sense that the intuitions underlying this dynamic interpretation are coherent with those underlying the ternary semantics of substructural logics, as witnessed by our quotes and citations from the substructural literature.

Other interpretations of the ternary relation. One interpretation, due to Barwise [15] and developed by Restall [60], takes worlds to be 'sites' or 'channels', a site being possibly a channel and a channel being possibly a site. If $x, y$ and $z$ are sites, $\mathcal{R} x y z$ reads as ' $x$ is a channel between $y$ and $z$ '. Hence, if $\varphi \supset \psi$ is true at channel $x$, it means that all sites $y$ and $z$ connected by channel $x$ are such that if $\varphi$ is information available in $y$, then $\psi$ is information available in $z$. Another similar interpretation due to Mares [47] adapts Israel and Perry's theory of information [55] to

\footnotetext{
${ }^{2}$ Here is Ramsey's footnote: "If two people are arguing 'If $p$, then $q$ ?' and are both in doubt as to $p$, they are adding $p$ hypothetically to their stock of knowledge and arguing on that basis about $q$; so that in a sense 'If $p$, $q$ ' and 'If $p, \neg q$ ' are contradictories. We can say that they are fixing their degree of belief in $q$ given $p$. If $p$ turns out false, these degrees of belief are rendered void. If either party believes not $p$ for certain, the question ceases to mean anything to him except as a question about what follows from certain laws or hypotheses.'[58 154-155]

${ }^{3}$ Note that Burgess [21] already proposed a ternary semantics for conditionals, but his truth conditions and his interpretation of the ternary relation were quite different from ours.
} 
the relational semantics. In this interpretation, worlds are situations in the sense of Barwise and Perry's situation semantics [14] and pieces of information - called infons - can carry information about other infons: an infon might carry the information that a red light on a mobile phone carries the information that the battery of the mobile phone is low. In this interpretation, the ternary relation $\mathcal{R}$ represents the informational links in situations: if there is an informational link in situation $x$ that says that an infon $\sigma$ carries the information that the infon $\pi$ also holds, then if $\mathcal{R} x y z$ holds and $y$ contains the infon $\sigma$, then $z$ contains the infon $\pi$. Other interpretations of the ternary relation have been proposed by Beall \& Al. [16], with a particular focus on their relation to conditionality. For more information on this topic the reader is invited to consult [49] which covers the material briefly reviewed in this paragraph.

\section{Update Logic}

In this section, we define our update logic. After introducing some mathematical definitions in Section 3.1, we motivate in Section 3.2 the introduction of three triples of logical connectives. These connectives generalize the triple $(\otimes, \supset, \subset)$ of substructural logics and will be given a semantics based on the cyclic permutations of the set $\{1,2,3\}$ in Section 3.3 .

\subsection{Preliminary Definitions}

The general definitions of this section will be used in the rest of the article.

Definition 5 (Logic). A logic is a triple $L:=\left(\mathcal{L}\left(\mathbb{P}, \mathrm{C}_{\varphi}\right), E, \models\right)$ where

1. $\mathcal{L}\left(\mathbb{P}, \mathrm{C}_{\varphi}\right)$ is a logical language defined as a set of well-formed expressions built from a set of logical (and structural) connectives $\mathrm{C}_{\varphi}$ and a set of propositional letters $\mathbb{P}$;

2. E is a class of pointed models or frames;

3. $\models$ is a satisfaction relation which relates in a compositional manner elements of $\mathcal{L}\left(\mathbb{P}, \mathrm{C}_{\varphi}\right)$ to models of $E$ by means of so-called truth conditions. [27].

Note that the above semantically-based definition of a logic is also used by French et Al.

Example 1. The triples $(\mathcal{L}(\mathbb{P}$, Sub $), \mathcal{E}, \Vdash)$ and $\left(\mathcal{L}\left(\mathbb{P}\right.\right.$, Sub $\left.\left._{-}\right), \mathcal{E}, \Vdash\right)$ are logics. We list in Figure 2 logics that we deem to be 'classical'. Display calculi for these logics can be found for example in [17, 78, 36].

Definition 6 (Expressiveness). Let two logics $\mathrm{L}=(\mathcal{L}, E, \models)$ and $\mathrm{L}^{\prime}=\left(\mathcal{L}^{\prime}, E, \models^{\prime}\right)$ be given (interpreted over the same class of models $E$ ). Let $\varphi \in \mathcal{L}$ and $\varphi^{\prime} \in \mathcal{L}^{\prime}$. We say that $\varphi$ is as expressive as $\varphi^{\prime}$ when $\{\mathcal{M} \in E \mid \mathcal{M} \models \varphi\}=\left\{\mathcal{M} \in E \mid \mathcal{M}=\varphi^{\prime}\right\}$. We say that $L$ has at least the same expressive power as $L^{\prime}$, denoted $L \geq L^{\prime}$, when for all $\varphi^{\prime} \in \mathcal{L}^{\prime}$, there is $\varphi \in \mathcal{L}$ such that $\varphi$ is as expressive as $\varphi^{\prime}$. When $L$ has at least the same expressive power as $L^{\prime}$ and vice versa, we say that $L$ and $\mathrm{L}^{\prime}$ have the same expressive power and we write it $\mathrm{L} \equiv \mathrm{L}^{\prime}$. Otherwise, $\mathrm{L}$ is strictly more expressive than $\mathrm{L}^{\prime}$ and we write it $\mathrm{L}>\mathrm{L}^{\prime}$.

Example 2. It holds that $(\mathcal{L}(\mathbb{P}$, Sub $), \mathcal{E}, \Vdash)>\left(\mathcal{L}\left(\mathbb{P}\right.\right.$, Sub $\left.\left._{-}\right), \mathcal{E}, \Vdash\right)$. 


\begin{tabular}{|c|c|c|c|c|}
\hline \multicolumn{2}{|c|}{ Models $E$} & \multirow{2}{*}{ Connectives $\mathrm{C}_{\varphi}$} & Logic $\left(\mathcal{L}\left(\mathbb{P}, \mathrm{C}_{\varphi}\right), E, \Vdash\right)$ \\
\hline$\sqsubseteq$ & $R$ & $\mathcal{R}$ & & \\
\hline$=$ & & & $\neg \wedge$ & Propositional Logic \\
$=$ & $\bullet$ & & $\neg \wedge \square$ & Modal Logic \\
$=$ & & $\bullet$ & $\otimes \supset \subset$ & Lambek Calculus \\
$=$ & $\bullet$ & $\bullet$ & $\neg \wedge \square \otimes \supset \subset$ & Modal Lambek Calculus \\
\hline
\end{tabular}

Figure 2: 'Classical' Logics

\subsection{Talking about Ternary Relations}

If we want to reason about updates, we must be able to express properties of updates. In other words, we need a language for talking about updates. Since we represent them by ternary relations, it seems natural to require that our language be able to express properties that relate what is true at each point of the ternary relations, that is, what is true at: 1. the initial situation (expressed by a formula $\varphi$ ), 2. the event occurring in this situation (expressed by a second formula $\chi$ ), 3. the resulting situation after the event has occurred (expressed by a third formula $\psi$ ):

$$
1, \varphi \longrightarrow 2, \chi \longrightarrow 3, \psi
$$

This leads us to the following general question: assume that we stand in one of these three time points $x$ (be it 1,2 or 3), what kind of property can we express and infer about the other time points $y$ and $z$ ? Here is a non-exhaustive list of the possible and most natural expressions that we would want to state:

(a) For all $y$, if $y$ satisfies $\varphi$ then for all $z, z$ satisfies $\psi$ : " $x \models \forall y \forall z(\varphi(y) \rightarrow \psi(z))$ ".

For example, in the initial state 1 , is it the case that any event satisfying $\chi$ will always lead to a state 3 satisfying $\psi$ ? Or, in state 3 , is it the case that before the occurrence of any event satisfying $\chi, \varphi$ held in all initial states 1 ?

(b) There exist $y$ and $z$ such that $y$ satisfies $\varphi$ and $z$ satisfies $\psi$ : " $x \models \exists y \exists z(\varphi(y) \wedge \psi(z))$ ".

For example, in state 1 , is it the case that there exists an event satisfying $\chi$ that may lead to a state where $\psi$ holds ? Or, in state 3 , is it possible that our current state might have been the result of an event satisfying $\chi$ in an initial state where $\varphi$ held?

(c) For all $y$ satisfying $\varphi$, there exists $z$ satisfying $\psi$ : " $x \models \forall y \exists z(\varphi(y) \rightarrow \psi(z))$ ".

For example, in state 1 , is it the case that any events satisfying $\chi$ may lead possibly to a state where $\psi$ holds ? Or, in state 3, is it the case that an event satisfying $\chi$ might have occurred so that any former situation before this event satisfied $\varphi$ ?

This list of expressions is obviously non-exhaustive. Providing formal tools that answer these kinds of questions leads to applications in artificial intelligence and theoretical computer 
science, and as it turns out, some of these questions have already been addressed in dynamic epistemic logic and other logical formalisms (see the companion article [7, Sect. 7.2] for more details and examples). Typically, most of the works about conditionals and belief dynamics deal with the first kind of statements (a) or (b). In fact, the conditionals $\supset$ and $\subset$ of substructural and relevance logics of the previous section are of the form (a), whereas the substructural connective $\otimes$ is of the form (b). The language that we will define will only deal with the first two kinds of expressions (a) and (b) (Section 3.3). This language is intended to capture the various conditionals and belief change operators which have been introduced in the philosophical and artificial intelligence literature. As shown in the companion article [7], it captures very well the operators of Dynamic Epistemic Logic.

\subsection{Syntax and Semantics of Update Logic}

We define formally formulas, structures and then consecutions (sometimes called sequents in the literature). This is an incremental definition and each of these objects is defined on the basis of the previous one. In the sequel we will view sets of formulas, sets of structures and sets of consecutions as logical languages.

Notation 1. In the first part of this article, we will use the following logical connectives $\operatorname{Con}_{\varphi}$ and structural connectives $\operatorname{Con}_{X}$ ( $\varphi$ will denote formulas and $X$ will denote structures):

$$
\begin{aligned}
\operatorname{Con}_{\varphi}: & =\left\{\top, \perp, \square, \diamond^{-}, \neg, \vee, \wedge, \otimes_{i}, \supset_{i}, \subset_{i} \mid i \in\{1,2,3\}\right\} \\
\operatorname{Con}_{X}: & =\left\{*, \bullet,,_{i} \mid i \in\{0,1,2,3\}\right\} .
\end{aligned}
$$

The connectives $\vee, \wedge, \otimes_{i}, \supset_{i}, \subset_{i},,_{o},,_{i}$ (where $i$ ranges over $\{1,2,3\}$ ) are binary connectives, $\square, \diamond^{-}, \neg, *, \bullet$ are unary connectives and $\top, \perp$ are nullary connectives. The structural connective, o will often simply be denoted, .

Definition 7 (Formula, structure and consecution).

- Let $\mathrm{C}_{\varphi} \subseteq \operatorname{Con}_{\varphi}$ be a non-empty set of logical connectives. The language associated to $C_{\varphi}$, denoted $\mathcal{L}\left(\mathbb{P}, \mathrm{C}_{\varphi}\right)$, is the language built compositionally from the connectives of $\mathrm{C}_{\varphi}$ and the set of propositional letters $\mathbb{P}$. Elements of the language $\mathcal{L}\left(\mathbb{P}, \mathrm{C}_{\varphi}\right)$ are called $\mathcal{L}\left(\mathbb{P}, C_{\varphi}\right)$-formulas and are generally denoted $\varphi, \chi, \psi, \ldots$

- Let $\mathrm{C}_{X} \subseteq$ Con $_{X}$ and $\mathrm{C}_{\varphi} \subseteq$ Con $_{\varphi}$ be non-empty sets of structural connectives and logical connectives. The set of structures associated to $C_{\varphi}$ and $C_{X}$, denoted $\mathcal{S}\left(\mathbb{P}, \mathrm{C}_{\varphi}, \mathrm{C}_{X}\right)$, is the language built compositionally from the structural connectives of $\mathrm{C}_{X}$ and the set $\mathcal{L}\left(\mathbb{P}, \mathrm{C}_{\varphi}\right)$. Elements of the language $\mathcal{S}\left(\mathbb{P}, \mathrm{C}_{\varphi}, \mathrm{C}_{X}\right)$ are called $\mathcal{S}\left(\mathbb{P}, C_{\varphi}, C_{X}\right)$-structures and are generally denoted $X, Y, Z, U, V, \ldots$

The structural connectives associated to $C_{\varphi}$, denoted $\operatorname{Struc}\left(\mathrm{C}_{\varphi}\right)$, is the set of structural connectives $\left\{*,,_{0}\right\}$ together with $\left\{,_{1},,_{2},,_{3}\right\}$ if $\mathrm{C}_{\varphi} \cap\left\{\otimes_{\mathrm{i}}, \supset_{\mathrm{i}}, \subset_{\mathrm{i}} \mid i \in\{1,2,3\}\right\} \neq$ $\emptyset$ and with $\{\bullet\}$ if $\mathrm{C}_{\varphi} \cap\left\{\square, \diamond^{-}\right\} \neq \emptyset$. We denote by $\mathcal{S}\left(\mathbb{P}, \mathrm{C}_{\varphi}\right)$ the set of all $\mathcal{S}\left(\mathbb{P}, \mathrm{C}_{\varphi}, \operatorname{Struc}\left(\mathrm{C}_{\varphi}\right)\right)$-structures. 
- Let $\mathrm{C}_{X} \subseteq$ Con $_{X}$ and $\mathrm{C}_{\varphi} \subseteq \operatorname{Con}_{\varphi}$ be non-empty sets of structural connectives and logical connectives. A $\mathcal{S}\left(\mathbb{P}, C_{\varphi}, C_{X}\right)$-consecution is an expression of the form $X \vdash Y, X \vdash$ or $\vdash Y$, where $X, Y \in \mathcal{S}\left(\mathbb{P}, \mathrm{C}_{\varphi}, \mathrm{C}_{X}\right)$. The $\mathcal{S}\left(\mathbb{P}, \mathrm{C}_{\varphi}, \mathrm{C}_{X}\right)$-structure $X$ is called the antecedent and the $\mathcal{S}\left(\mathbb{P}, \mathrm{C}_{\varphi}, \mathrm{C}_{X}\right)$-structure $Y$ is called the consequent. We denote by $\mathcal{C}\left(\mathrm{C}_{\varphi}\right)$ the set of all $\mathcal{S}\left(\mathbb{P}, \mathrm{C}_{\varphi}, \operatorname{Struc}\left(\mathrm{C}_{\varphi}\right)\right)$-consecutions.

The connective $\rightarrow$ (standing for the material implication) is defined by the following abbreviation: $(\varphi \rightarrow \psi):=(\neg \varphi \vee \psi)$. To avoid any ambiguity, every occurence of any binary connective is surrounded by brackets.

Example 3. If $\mathrm{C}_{\varphi}=\left\{\neg, \wedge, \otimes_{3}, \supset_{1}, \subset_{2}\right\}$, then the language $\mathcal{L}\left(\mathbb{P}, \mathrm{C}_{\varphi}\right)$ is defined by the following grammar in $\mathrm{BNF}$, where $p$ ranges over $\mathbb{P}$ :

$$
\mathcal{L}\left(\mathbb{P}, \mathrm{C}_{\varphi}\right): \quad \varphi::=p|\neg \varphi|(\varphi \wedge \varphi)\left|\left(\varphi \otimes_{3} \varphi\right)\right|\left(\varphi \supset_{1} \varphi\right) \mid\left(\varphi \subset_{2} \varphi\right)
$$

Then, we have that $\operatorname{Struc}\left(\mathrm{C}_{\varphi}\right)=\left\{*,,_{0},,_{\mathrm{i}} \mid i \in\{1,2,3\}\right\}$. So, the language $\mathcal{S}\left(\mathbb{P}, \mathrm{C}_{\varphi}\right):=$ $\mathcal{S}\left(\mathbb{P}, \mathrm{C}_{\varphi}, \operatorname{Struc}\left(\mathrm{C}_{\varphi}\right)\right)$ is defined by the following grammar in $\mathrm{BNF}$, where $\varphi$ ranges over $\mathcal{L}\left(\mathbb{P}, \mathrm{C}_{\varphi}\right)$ and $i$ ranges over $\{1,2,3\}$ :

$$
\mathcal{S}\left(\mathbb{P}, \mathrm{C}_{\varphi}\right): X \quad:=\varphi|* X|\left(X,{ }_{0} X\right) \mid\left(X,{ }_{\mathrm{i}} X\right)
$$

Notation 2. To save parenthesis, we use the following ranking of binding strength: $\otimes_{i}, \supset_{i}, \subset_{i}$ $, \wedge, \vee, \rightarrow$ (where i ranges over $\{1,2,3\})$. For example, $\square \neg p \wedge q \rightarrow \neg r \otimes_{3}$ s stands for $((\square(\neg p)) \wedge$ $q) \rightarrow\left((\neg r) \otimes_{3} s\right)$ (additional brackets have been added for the unary connectives $\square$ and $\neg$, even if they are not needed and will not appear in any formula anyway). For every binary connective $\star$, we use the following notation: $X_{1} \star \ldots \star X_{n}:=\left(\left(\ldots\left(X_{1} \star \ldots \star X_{n-2}\right) \star X_{n-1}\right) \star X_{n}\right)$. For example, $\varphi_{1} \vee \ldots \vee \varphi_{n}:=\left(\left(\ldots\left(\varphi_{1} \vee \ldots \vee \varphi_{n-2}\right) \vee \varphi_{n-1}\right) \vee \varphi_{n}\right)$ and $X_{1}, \ldots, X_{n}:=$ $\left(\left(\ldots\left(X_{1}, \ldots, X_{n-2}\right), X_{n-1}\right), X_{n}\right)$. Moreover, if $\Gamma:=\left\{\varphi_{1}, \ldots, \varphi_{n}\right\}$ is a finite set of formulas and $\star$ is a binary connective over formulas, we use the following notation: $\star \Gamma:=\varphi_{1} \star \ldots \star \varphi_{n}$. For example, $\bigvee \Gamma:=\varphi_{1} \vee \ldots \vee \varphi_{n}$ and $\bigwedge \Gamma:=\varphi_{1} \wedge \ldots \wedge \varphi_{n}$

Definition 8 (Update logic). Let $E$ be an arbitrary set of three elements. For each $i \in\{1,2,3\}$, we define the permutations $\sigma_{i}: E^{3} \mapsto E^{3}$ as follows: for all $x, y, z \in E$,

$$
\sigma_{1}(x, y, z)=(x, y, z) \quad \sigma_{2}(x, y, z)=(z, x, y) \quad \sigma_{3}(x, y, z)=(y, z, x) .
$$

- We define the evaluation relation $\Vdash \subseteq \mathcal{E} \times \mathcal{L}\left(\mathbb{P}, \operatorname{Con}_{\varphi}\right)$ inductively as follows. Let $(\mathcal{M}, x) \in \mathcal{E}$ be a pointed substructural model and let $\varphi \in \mathcal{L}\left(\mathbb{P}, \operatorname{Con}_{\varphi}\right)$. The truth conditions for the connectives $\square, \diamond^{-}, \wedge, \vee, \perp, \top$ are defined like in Definition 4 . The truth condition for the Boolean negation is defined as follows:

$$
\mathcal{M}, x \Vdash \neg \varphi \quad \text { iff } \quad \text { it is not the case that } \mathcal{M}, x \| \varphi .
$$


The truth conditions for the connectives $\otimes_{\mathrm{i}}, \supset_{\mathrm{i}}, \subset_{\mathrm{i}}$ are defined as follows: for all $i \in$ $\{1,2,3\}$, we have that

$$
\begin{array}{rll}
\mathcal{M}, x \Vdash \varphi \otimes_{\mathrm{i}} \psi \quad \text { iff } & \text { there are } y, z \in \mathcal{P} \text { such that } \sigma_{i}(x, y, z) \in \mathcal{R}, \\
& \mathcal{M}, y \| \varphi \text { and } \mathcal{M}, z \| \psi ; \\
\mathcal{M}, x \| \varphi \supset_{\mathrm{i}} \psi \quad \text { iff } \quad & \text { for all } y, z \in \mathcal{P} \text { such that } \sigma_{i}(x, y, z) \in \mathcal{R}, \\
& \text { if } \mathcal{M}, y \| \varphi \text { then } \mathcal{M}, z \Vdash \psi ; \\
\mathcal{M}, x \Vdash \varphi \subset_{\mathrm{i}} \psi \quad \text { iff } \quad & \text { for all } y, z \in \mathcal{P} \text { such that } \sigma_{i}(x, y, z) \in \mathcal{R}, \\
& \text { if } \mathcal{M}, z \| \psi \text { then } \mathcal{M}, y \Vdash \varphi .
\end{array}
$$

- We extend the scope of the evaluation relation $\Vdash$ simultaneously in two different ways in order to also relate points to $\mathcal{S}\left(\mathbb{P}, \operatorname{Con}_{\varphi}\right)$-structures. The antecedent evaluation relation $\|^{A} \subseteq \mathcal{E} \times \mathcal{S}\left(\mathbb{P}, \operatorname{Con}_{\varphi}\right)$ is defined inductively as follows: for all $i \in\{1,2,3\}$,

$$
\begin{array}{lll}
\mathcal{M}, x \|^{A} \varphi & \text { iff } & \mathcal{M}, x \| \varphi ; \\
\mathcal{M}, x \|^{A} * X & \text { iff } & \text { it is not the case that } \mathcal{M}, x \|^{K} X ; \\
\mathcal{M}, x \|^{A} \bullet X & \text { iff } & \text { there is } y \in \mathcal{M} \text { such that } y R x \\
& & \text { and it holds that } \mathcal{M}, y \|^{A} X ; \\
\mathcal{M}, x \|^{A} X, Y & \text { iff } & \mathcal{M}, x \|^{A} X \text { and } \mathcal{M}, x \|^{A} Y ; \\
\mathcal{M}, x \|^{A} X,{ }_{\mathrm{i}} Y & \text { iff } & \text { there are } y, z \in \mathcal{M} \text { such that } \sigma_{i}(x, y, z) \in \mathcal{R}, \\
& & \mathcal{M}, y \|^{A} X \text { and } \mathcal{M}, z \|^{A} Y .
\end{array}
$$

The consequent evaluation relation $\|^{K} \subseteq \mathcal{E} \times \mathcal{S}\left(\mathbb{P}, \operatorname{Con}_{\varphi}\right)$ is defined inductively as follows: for all $i \in\{1,2,3\}$,

$$
\begin{array}{lll}
\mathcal{M}, x \| \frac{K}{K} \varphi & \text { iff } & \mathcal{M}, x \| \varphi ; \\
\mathcal{M}, x \| \frac{K}{*} * X & \text { iff } & \text { it is not the case that } \mathcal{M}, x \|^{A} X ; \\
\mathcal{M}, x \| \underline{K} \bullet X & \text { iff } & \text { for all } y \in \mathcal{M} \text { such that } x R y, \\
& & \text { it holds that } \mathcal{M}, y \|^{K} X ; \\
\mathcal{M}, x \| \underline{K} X, Y & \text { iff } & \mathcal{M}, x \|^{K} X \text { or } \mathcal{M}, x \|^{K} Y ; \\
\mathcal{M}, x \| \underline{K} X,{ }_{i} Y & \text { iff } & \text { for all } y, z \in \mathcal{M} \text { such that } \sigma_{i}(x, y, z) \in \mathcal{R}, \\
& & \mathcal{M}, y \|^{K} X \text { or } \mathcal{M}, z \|^{K} Y .
\end{array}
$$

- We extend the scope of the relation $\|$ to also relate points to $\mathcal{S}\left(\mathbb{P}, \operatorname{Con}_{\varphi}\right)$-consecutions. Depending on the form of the $\mathcal{S}\left(\mathbb{P}, \operatorname{Con}_{\varphi}\right)$-consecution, that is, whether it is of the form $X \vdash Y, \vdash Y$ or $X \vdash$, we have:

$$
\begin{aligned}
& \mathcal{M}, x \Vdash X \vdash Y \quad \text { iff } \quad \text { if } \mathcal{M}, x \|^{A} X \text {, then } \mathcal{M}, x \|^{K} Y \text {; } \\
& \mathcal{M}, x \Vdash \vdash Y \quad \text { iff } \quad \mathcal{M}, x \|^{K} Y \text {; } \\
& \mathcal{M}, x \Vdash X \vdash \quad \text { iff } \quad \text { it is not the case that } \mathcal{M}, x \|^{A} X \text {. }
\end{aligned}
$$

So, for all $\mathrm{C}_{\varphi} \subseteq \operatorname{Con}_{\varphi}$, the triples $\left(\mathcal{L}\left(\mathbb{P}, \mathrm{C}_{\varphi}\right), \mathcal{E}, \|\right),\left(\mathcal{S}\left(\mathbb{P}, \mathrm{C}_{\varphi}\right), \mathcal{E}, \|^{A}\right)$, $\left(\mathcal{S}\left(\mathbb{P}, \mathrm{C}_{\varphi}\right), \mathcal{E}, \|^{K}\right)$ and $\left(\mathcal{C}\left(\mathrm{C}_{\varphi}\right), \mathcal{E}, \Vdash\right)$ are logics (as defined in Definition 5). The triple $\left(\mathcal{L}\left(\mathbb{P}, \operatorname{Con}_{\varphi}\right), \mathcal{E}, \Vdash\right)$ is also a logic, called update logic. 
Our semantics is based on the cyclic permutations of the set $\{1,2,3\}$. In group theory, a cyclic permutation $\sigma$ on a finite set $S$ is a bijection on $S$ such that $\sigma\left(s_{i}\right)=s_{i+1}$ for $i<n$ and $\sigma\left(s_{n}\right)=s_{1}$, where $S=\left\{s_{1}, \ldots, s_{n}\right\}$, and in that case we write $\sigma:=$ $\left(s_{1} \ldots s_{n}\right)$. For the set $\{1,2,3\}$, there are two cyclic permutations: $\tau:=(123)$ and $\rho:=(132)$ (and if $I d$ denotes the identity permutation and $\circ$ the composition of applications, then $(\{\tau, \rho, I d\}, \circ)$ is a group). Then, one should note that for all $x_{1}, x_{2}, x_{3} \in E$, we have that $\sigma_{1}\left(x_{1}, x_{2}, x_{3}\right)=\left(x_{I d(1)}, x_{I d(2)}, x_{I d(3)}\right), \sigma_{2}\left(x_{1}, x_{2}, x_{3}\right)=\left(x_{\tau(1)}, x_{\tau(2)}, x_{\tau(3)}\right)$ and $\sigma_{3}\left(x_{1}, x_{2}, x_{3}\right)=\left(x_{\rho(1)}, x_{\rho(2)}, x_{\rho(3)}\right)$.

Remark 2. We do not introduce the truth constants $\mathbf{t}$ and $\mathbf{f}$, because we allow empty antecedents and consequents in our consecutions (we provide a specific semantics for that). This is not usual for display calculi, ever since Belnap's seminal paper [17, Footnote 2] where empty antecedents and consequents are not allowed. On the other hand, this feature will allow us to refrain from introducing empty structural connectives $\mathbf{I}$.

Remark 3. Usually, one gives a semantics to structures by means of mappings that translate structures into formulas, like in Proposition 1. We prefer to give semantics to structures directly by interpreting them over substructural models, because we cannot always provide an equivalent formula for a structure, depending on the logical language we consider. In fact, all our display calculi contain the structural connectives $*$ and ${ }_{0}$, but the corresponding Boolean negation $\neg$ and conjunction/disjunction $\wedge / \vee$ are not always present in the logical language (this is especially the case for substructural logics which usually do not contain Boolean negation). Moreover, giving semantics to structures will also allow us to obtain strong completeness results for display calculi.

Spelling out the truth conditions for the connectives $\otimes_{\mathrm{i}}, \supset_{\mathrm{i}}$ and $\subset_{\mathrm{i}}$ for $i \in\{1,2,3\}$, we obtain the expressions of Figure 3 . The indices 1, 2 and 3 of our connectives indicate when formulas are evaluated. The connectives $\supset_{1}, \subset_{1}$ and $\otimes_{1}$ express properties of updates before the event, the connectives $C_{2}, \supset_{2}$ and $\otimes_{2}$ properties during the event and the connectives $\supset_{3}, \subset_{3}$ and $\otimes_{3}$ properties after the event. Typically, the formula $\varphi$ deals with the initial situation, the formula $\chi$ deals with the event and the formula $\psi$ deals with the final situation. The direction of the arrow $(\subset$ or $\supset$ ) indicates the conditional direction in which the formula should be read. For example, the formula $\psi \supset_{2} \varphi$ tells us that it should be evaluated during an event (2) and reads as "if the final situation will satisfy $\psi$ then the initial situation must necessarily satisfy $\varphi$ ", whereas $\psi \subset_{2} \varphi$ reads as "if the initial situation satisfies $\varphi$ then the final situation will necessarily satisfy $\psi$ ". The formula $\chi \supset_{1} \psi$ reads as " $\psi$ will hold after the occurrence of any events satisfying $\chi$ " and the formula $\varphi \subset_{3} \chi$ reads as " $\varphi$ held before the occurrence of any events satisfying $\chi$ ". The connectives $\otimes_{1}, \otimes_{2}, \otimes_{3}$ are of the form (b) and the connectives $\supset_{1}, \subset_{1}, \supset_{2}, \subset_{2}$ $, \supset_{3}, \subset_{3}$ are of the form (a) (see Page 10). Note that the classical substructural connectives $\otimes, \supset$ and $\subset$ of the previous section correspond to our connectives $\otimes_{3}, \supset_{1}$ and $\subset_{2}$. So, our language $\mathcal{L}\left(\mathbb{P}, \mathrm{Con}_{\varphi}\right)$ extends the language $\mathcal{L}\left(\mathbb{P}, \mathrm{Sub}_{-}\right)$of substructural logics presented in Section 2.1 and the logic $\left(\mathcal{L}\left(\mathbb{P}, \operatorname{Con}_{\varphi}\right), \mathcal{E}, \Vdash\right)$ is therefore at least as expressive as $\left(\mathcal{L}\left(\mathbb{P}\right.\right.$, Sub$\left.\left._{-}\right), \mathcal{E}, \Vdash-\right)$. Proposition 1 below shows that $\left(\mathcal{L}\left(\mathbb{P}, \operatorname{Con}_{\varphi}\right), \mathcal{E}, \Vdash\right)$ is in fact strictly more expressive than $\left(\mathcal{L}\left(\mathbb{P}\right.\right.$, Sub$\left.\left._{-}\right), \mathcal{E}, \Vdash\right)$. 


\begin{tabular}{|c|c|c|}
\hline $\begin{array}{l}\mathcal{M}, x \Vdash \chi \otimes_{1} \psi \\
\mathcal{M}, x \Vdash \chi \supset_{1} \psi \\
\mathcal{M}, x \Vdash \chi \subset_{1} \psi \\
\mathcal{M}, x \Vdash \psi \otimes_{2} \varphi \\
\mathcal{M}, x \Vdash \psi \supset_{2} \varphi \\
\mathcal{M}, x \Vdash \psi \subset_{2} \varphi \\
\mathcal{M}, x \Vdash \varphi \otimes_{3} \chi \\
\mathcal{M}, x \Vdash \varphi \supset_{3} \chi \\
\mathcal{M}, x \Vdash \varphi \subset_{3} \chi\end{array}$ & iff & $\begin{array}{l}\text { there are } y, z \in \mathcal{P} \text { such that } \mathcal{R} x y z, \\
\mathcal{M}, y \Vdash \chi \text { and } \mathcal{M}, z \Vdash \psi ; \\
\text { for all } y, z \in \mathcal{P} \text { such that } \mathcal{R} x y z, \\
\text { if } \mathcal{M}, y \Vdash \chi \text { then } \mathcal{M}, z \Vdash \psi ; \\
\text { for all } y, z \in \mathcal{P} \text { such that } \mathcal{R} x y z, \\
\text { if } \mathcal{M}, z \Vdash \psi \text { then } \mathcal{M}, y \Vdash \chi ; \\
\text { there are } y, z \in \mathcal{P} \text { such that } \mathcal{R} z x y, \\
\mathcal{M}, z \Vdash \varphi \text { and } \mathcal{M}, y \Vdash \psi ; \\
\text { for all } y, z \in \mathcal{P} \text { such that } \mathcal{R} z x y, \\
\text { if } \mathcal{M}, y \Vdash \psi \text { then } \mathcal{M}, z \Vdash \varphi ; \\
\text { for all } y, z \in \mathcal{P} \text { such that } \mathcal{R} z x y, \\
\text { if } \mathcal{M}, z \Vdash \varphi \text { then } \mathcal{M}, y \Vdash \psi ; \\
\text { there are } y, z \in \mathcal{P} \text { such that } \mathcal{R} y z x, \\
\mathcal{M}, y \Vdash \varphi \text { and } \mathcal{M}, z \Vdash \chi ; \\
\text { for all } y, z \in \mathcal{P} \text { such that } \mathcal{R} y z x, \\
\text { if } \mathcal{M}, y \Vdash \varphi \text { then } \mathcal{M}, z \Vdash \chi ; \\
\text { for all } y, z \in \mathcal{P} \text { such that } \mathcal{R} y z x, \\
\text { if } \mathcal{M}, z \Vdash \chi \text { then } \mathcal{M}, y \Vdash \varphi \text {. }\end{array}$ \\
\hline
\end{tabular}

Figure 3: Spelling out the Truth Conditions

Proposition 1. We define inductively the following translations $\tau_{1}$ and $\tau_{2}$ from $\mathcal{S}\left(\mathbb{P}, \operatorname{Con}_{\varphi}\right)-$ structures to $\mathcal{L}\left(\mathbb{P}, \mathrm{Con}_{\varphi}\right)$-formulas: for all $i \in\{1,2,3\}$,

$$
\begin{aligned}
\tau_{1}(\varphi) & :=\varphi \\
\tau_{1}(* X) & :=\neg \tau_{2}(X) \\
\tau_{1}(\bullet X) & :=\diamond^{-} \tau_{1}(X) \\
\tau_{1}(X, Y) & :=\tau_{1}(X) \wedge \tau_{1}(Y) \\
\tau_{1}(X, Y) & :=\tau_{1}(X) \otimes_{i} \tau_{1}(Y)
\end{aligned}
$$

$$
\begin{aligned}
\tau_{2}(\varphi) & :=\varphi \\
\tau_{2}(* X) & :=\neg \tau_{1}(X) \\
\tau_{2}(\bullet X) & :=\square \tau_{2}(X) \\
\tau_{2}(X, Y) & :=\tau_{2}(X) \vee \tau_{2}(Y) \\
\tau_{2}(X, Y) & :=\neg\left(\neg \tau_{2}(X) \otimes_{i} \neg \tau_{2}(Y)\right)
\end{aligned}
$$

Then, for all pointed substructural models $(\mathcal{M}, x)$, all $X \in \mathcal{S}\left(\mathbb{P}\right.$, Con $\left._{\varphi}\right)$,

$$
\begin{gathered}
\mathcal{M}, x \|^{A} X \quad \text { iff } \quad \mathcal{M}, x \| \tau_{1}(X) \\
\mathcal{M}, x \|^{K} X \quad \text { iff } \quad \mathcal{M}, x \Vdash \tau_{2}(X)
\end{gathered}
$$

Moreover, the following hold:

- $\left(\mathcal{L}\left(\mathbb{P}, C_{\varphi}\right), \mathcal{E}, \Vdash\right)>(\mathcal{L}(\mathbb{P}$, Sub $), \mathcal{E}, \Vdash)$, where $C_{\varphi}:=\operatorname{Con}_{\varphi}-\{\neg\}$;

- $\left(\mathcal{L}\left(\mathbb{P}, \operatorname{Con}_{\varphi}\right), \mathcal{E}, \Vdash\right) \equiv\left(\mathcal{S}\left(\mathbb{P}, \operatorname{Con}_{\varphi}\right), \mathcal{E}, \|^{A}\right) \equiv\left(\mathcal{S}\left(\mathbb{P}, \operatorname{Con}_{\varphi}\right), \mathcal{E}, \|^{K}\right)$. 
Proof. The proofs of Expressions (3) and (4) is by induction on $X$. Now, we prove the other items separately.

- The $\operatorname{logic}\left(\mathcal{L}\left(\mathbb{P}, \operatorname{Con}_{\varphi}\right), \mathcal{E}, \Vdash\right)$ has at least the same expressive power as $\left(\mathcal{L}\left(\mathbb{P}\right.\right.$, Sub $\left.\left._{-}\right), \mathcal{E}, \Vdash\right)$, because $\mathcal{L}\left(\mathbb{P}\right.$, Sub $\left._{-}\right) \subseteq \mathcal{L}\left(\mathbb{P}, \operatorname{Con}_{\varphi}\right)$. To prove that $\left(\mathcal{L}\left(\mathbb{P}, \operatorname{Con}_{\varphi}\right), \mathcal{E}, \Vdash\right)$ is in fact strictly more expressive than $\left(\mathcal{L}\left(\mathbb{P}\right.\right.$, Sub $\left.\left._{-}\right), \mathcal{E}, \Vdash\right)$, we consider the substructural models $\mathcal{M}:=(\mathcal{P}, R, \mathcal{R}, \mathcal{I})$ and $\mathcal{M}^{\prime}:=\left(\mathcal{P}^{\prime}, R^{\prime}, \mathcal{R}^{\prime}, \mathcal{I}^{\prime}\right)$ defined as follows: $\mathcal{P}=\mathcal{P}^{\prime}:=\left(\left\{w_{0}, w_{1}, \ldots, w_{6}\right\},=\right), R=R^{\prime}:=\emptyset, \mathcal{R}=\mathcal{R}^{\prime}:=$ $\left\{\left(w_{0}, w_{1}, w_{3}\right),\left(w_{0}, w_{1}, w_{4}\right),\left(w_{0}, w_{2}, w_{5}\right),\left(w_{0}, w_{2}, w_{6}\right)\right\}, \mathcal{I}\left(w_{0}\right)=\mathcal{I}\left(w_{3}\right)=\mathcal{I}\left(w_{4}\right)=$ $\mathcal{I}\left(w_{6}\right):=\{p\}, \mathcal{I}\left(w_{1}\right)=\mathcal{I}\left(w_{2}\right)=\mathcal{I}\left(w_{5}\right):=\emptyset$ and $\mathcal{I}^{\prime}\left(w_{0}\right)=\mathcal{I}^{\prime}\left(w_{3}\right)=\mathcal{I}^{\prime}\left(w_{6}\right):=$ $\{p\}, \mathcal{I}^{\prime}\left(w_{1}\right)=\mathcal{I}^{\prime}\left(w_{2}\right)=\mathcal{I}^{\prime}\left(w_{3}\right)=\mathcal{I}^{\prime}\left(w_{5}\right):=\emptyset$. We are going to show that $\left(\mathcal{M}, w_{0}\right)$ and $\left(\mathcal{M}^{\prime}, w_{0}^{\prime}\right)$ satisfy the same formulas of $\mathcal{L}\left(\mathbb{P}\right.$, Sub $\left._{-}\right)$but not the same formulas of $\mathcal{L}\left(\mathbb{P}, \operatorname{Con}_{\varphi}\right)$. This will prove that $\mathcal{L}\left(\mathbb{P}, \operatorname{Con}_{\varphi}\right)$ is strictly more expressive than $\mathcal{L}\left(\mathbb{P}\right.$, Sub $\left._{-}\right)$. To show that $\left(\mathcal{M}, w_{0}\right)$ and $\left(\mathcal{M}^{\prime}, w_{0}^{\prime}\right)$ make the same formulas of $\mathcal{L}\left(\mathbb{P}, \mathrm{Sub}_{-}\right)$true, it suffices to show that there exists a directed bisumulation $(\Rightarrow, \Leftarrow)$ between $\left(\mathcal{M}, w_{0}\right)$ and $\left(\mathcal{M}^{\prime}, w_{0}^{\prime}\right)$ such that $w_{0} \Rightarrow w_{0}^{\prime}$ and $w_{0} \Leftarrow w_{0}^{\prime}$ (see [42] or [70] for a definition of directed bisimulation). We define the directed bisimulation $(\Rightarrow, \Leftarrow)$ as follows: $\Rightarrow:=\left\{\left(w_{i}, w_{i}^{\prime}\right) \mid i \neq 4\right\} \cup\left\{\left(w_{4}, w_{3}^{\prime}\right)\right\}$ and $\Leftarrow:=\left\{\left(w_{i}, w_{i}^{\prime}\right) \mid i \neq 4\right\} \cup\left\{\left(w_{4}, w_{3}^{\prime}\right),\left(w_{4}, w_{4}^{\prime}\right)\right\}$. Then, by [70, Fact 12.12], we have that for all $\varphi \in \mathcal{L}\left(\mathbb{P}\right.$, Sub $\left._{-}\right)$, it holds that $\mathcal{M}, w_{0} \Vdash \varphi$ if, and only if, $\mathcal{M}^{\prime}, w_{0}^{\prime} \Vdash \varphi$. However, as one can easily check, we also have that $\mathcal{M}, w_{0} \Vdash\left(p \subset_{2} \top\right) \otimes_{1} \top$ but it is not the case that $\mathcal{M}^{\prime}, w_{0}^{\prime} \Vdash\left(p \subset_{2} \top\right) \otimes_{1} \top$. Therefore, $\left(\mathcal{L}\left(\mathbb{P}, \operatorname{Con}_{\varphi}\right), \mathcal{E}, \Vdash\right)$ is strictly more expressive than $\left(\mathcal{L}\left(\mathbb{P}\right.\right.$, Sub $\left.\left._{-}\right), \mathcal{E}, \Vdash\right)$.

- The logics $\left(\mathcal{S}\left(\mathbb{P}, \operatorname{Con}_{\varphi}\right), \mathcal{E}, \|^{A}\right)$ and $\left(\mathcal{S}\left(\mathbb{P}, \operatorname{Con}_{\varphi}\right), \mathcal{E}, \|^{K}\right)$ have at least the same expressive power as the logic $\left(\mathcal{L}\left(\mathbb{P}, \operatorname{Con}_{\varphi}\right), \mathcal{E}, \Vdash\right)$ because $\mathcal{L}\left(\mathbb{P}, \operatorname{Con}_{\varphi}\right) \subseteq \mathcal{S}\left(\mathbb{P}, \operatorname{Con}_{\varphi}\right)$ and the definitions of $\|,\|^{A}$ and $\|^{K}$ coincide on $\mathcal{L}\left(\mathbb{P}, \operatorname{Con}_{\varphi}\right)$. Reciprocally, to prove that $\left(\mathcal{L}\left(\mathbb{P}, \operatorname{Con}_{\varphi}\right), \mathcal{E}, \Vdash\right)$ has at least the same expressive power as $\left(\mathcal{S}\left(\mathbb{P}, \operatorname{Con}_{\varphi}\right), \mathcal{E}, \|^{A}\right)$ and $\left(\mathcal{S}\left(\mathbb{P}, \operatorname{Con}_{\varphi}\right), \mathcal{E}, \|^{K}\right)$, we use the mappings of Proposition 1 Expressions (3) and (4) prove that $\left(\mathcal{L}\left(\mathbb{P}, \operatorname{Con}_{\varphi}\right), \mathcal{E}, \Vdash\right)$ has at least the same expressive power as $\left(\mathcal{S}\left(\mathbb{P}, \operatorname{Con}_{\varphi}\right), \mathcal{E}, \|^{A}\right)$ and $\left(\mathcal{S}\left(\mathbb{P}, \operatorname{Con}_{\varphi}\right), \mathcal{E}, \|^{K}\right)$.

\section{A Display Calculus for Update Logic}

Extending Gentzen's original sequent calculi with modalities has turned out over the years to be difficult. Many of the interesting theoretical properties of sequent calculi are lost when one adds modalities (see for example Poggiolesi [56, Chapter 1] for more details). A number of methods have been proposed to overcome these difficulties: display calculi, labelled sequents, tree hypersequents (see Poggiolesi and Restall [57] for an accessible introduction to these different sorts of calculi). In this section, we provide a display calculus for our update logic. As we will see, this display calculus will be a generalization of the display calculus for modal logic introduced by Wansing [76]. In the companion article [7], we provide a sequent calculus for update logic which turns out to be a generalization of the non-associative Lambek calculus [43, 44]. 


\subsection{Preliminary Definitions}

The general definitions of this section will be used in the rest of the article.

Definition 9 (Truth, validity, logical consequence). Let $\mathrm{L}=(\mathcal{L}, E, \models)$ be a logic. Let $\mathcal{M} \in E$, $\Gamma \subseteq \mathcal{L}$ and $\varphi \in \mathcal{L}$. We write $\mathcal{M} \models \Gamma$ when for all $\psi \in \Gamma$, we have $\mathcal{M} \models \psi$. Then, we say that

- $\varphi$ is true (satisfied) at $\mathcal{M}$ or $\mathcal{M}$ is a model of $\varphi$ when $\mathcal{M} \models \varphi$;

- $\varphi$ is a logical consequence of $\Gamma$, denoted $\Gamma \models\llcorner\varphi$, when for all $\mathcal{M} \in E$, if $\mathcal{M} \models \Gamma$ then $\mathcal{M}=\varphi$;

- $\varphi$ is valid, denoted $\models \mathrm{L} \varphi$, when for all models $\mathcal{M} \in E$, we have $\mathcal{M} \models \varphi$.

Definition 10 (Conservativity). Let $\mathrm{L}=(\mathcal{L}, E, \models)$ and $\mathrm{L}^{\prime}=\left(\mathcal{L}^{\prime}, E^{\prime}, \models^{\prime}\right)$ be two logics such that $\mathcal{L} \subseteq \mathcal{L}^{\prime}$. We say that $\mathrm{L}^{\prime}$ is a conservative extension of $\mathrm{L}$ when $\left\{\varphi \in \mathcal{L} \mid \models_{\mathrm{L}} \varphi\right\}=$ $\mathcal{L} \cap\left\{\varphi^{\prime} \in \mathcal{L}^{\prime} \mid \models_{\mathrm{L}^{\prime}}^{\prime} \varphi^{\prime}\right\}$.

Our definition of a proof system and of an inference rule is taken from Mendelson [50].

Definition 11 (Proof system and sequent calculus). Let $\mathrm{L}=(\mathcal{L}, E, \models)$ be a logic. A proof system $\mathcal{P}$ for $\mathcal{L}$ is a set of elements of $\mathcal{L}$ called axioms and a set of inference rules. Most often, one can effectively decide whether a given element of $\mathcal{L}$ is an axiom. To be more precise, an inference rule $R$ in $\mathcal{L}$ is a relation among elements of $\mathcal{L}$ such that there is a unique $l \in \mathbb{N}^{*}$ such that, for all $\varphi, \varphi_{1}, \ldots, \varphi_{l} \in \mathcal{L}$, one can effectively decide whether $\left(\varphi_{1}, \ldots, \varphi_{l}, \varphi\right) \in R$. The elements $\varphi_{1}, \ldots, \varphi_{l}$ are called the premises and $\varphi$ is called the conclusion and we say that $\varphi$ is a direct consequence of $\varphi_{1}, \ldots, \varphi_{l}$ by virtue of $R$. Let $\Gamma \subseteq \mathcal{L}$ and let $\varphi \in \mathcal{L}$. We say that $\varphi$ is provable (from $\Gamma$ ) in $\mathcal{P}$ or a theorem of $\mathcal{P}$, denoted $\vdash_{\mathcal{P}} \varphi\left(\right.$ resp. $\Gamma \vdash_{\mathcal{P}} \varphi$ ), when there is a proof of $\varphi$ (from $\Gamma$ ) in $\mathcal{P}$, that is, a finite sequence of formulas ending in $\varphi$ such that each of these formulas is:

1. either an instance of an axiom of $\mathcal{P}$ (or a formula of $\Gamma$ );

2. or the direct consequence of preceding formulas by virtue of an inference rule $R$.

If $\mathcal{S}$ is a set of $\mathcal{L}$-consecutions, this set $\mathcal{S}$ can be viewed as a logical language. Then, we call sequent calculus for $\mathcal{S}$ a proof system for $\mathcal{S}$.

Definition 12 (Soundness and completeness). Let $L=(\mathcal{L}, E, \models)$ be a logic. Let $\mathcal{P}$ be a proof system for $\mathcal{L}$. Then,

- $\mathcal{P}$ is sound for the logic $L$ when for all $\varphi \in \mathcal{L}$, if $\vdash_{\mathcal{P}} \varphi$, then $\models_{\llcorner} \varphi$.

- $\mathcal{P}$ is (strongly) complete for the logic $\mathrm{L}$ when for all $\varphi \in \mathcal{L}$ (and all $\Gamma \subseteq \mathcal{L}$ ), if $\models_{\mathrm{L}} \varphi$, then $\vdash_{\mathcal{P}} \varphi$ (resp. if $\Gamma \models_{\llcorner} \varphi$, then $\Gamma \vdash_{\mathcal{P}} \varphi$ ).

Because a proof is a finite sequence of formulas, soundness for the logic $L$ coincides with 'strong' soundness for the logic L, i.e. for all $\varphi \in \mathcal{L}$ and all $\Gamma \subseteq \mathcal{L}$, if $\Gamma \vdash_{\mathcal{P}} \varphi$, then $\Gamma \models_{\llcorner} \varphi$. 


\subsection{A Generalized Modal Display Calculus}

In this section, we introduce a display calculus for our update logic. It generalizes the modal display calculus of Wansing [76].

Definition 13 (Display calculus $\operatorname{UL}\left(\mathrm{C}_{\varphi}\right)$ ). Let $\mathrm{C}_{\varphi} \subseteq \operatorname{Con}_{\varphi}$. The display calculus for $\mathcal{C}\left(C_{\varphi}\right)$, denoted $\operatorname{UL}\left(\mathrm{C}_{\varphi}\right)$, is the display calculus containing the rules of Figure 5 mentioning the logical connectives of $\mathrm{C}_{\varphi}$ and the rules of Figure 4 mentioning the structural connectives of $\operatorname{Struc}\left(\mathrm{C}_{\varphi}\right)$ (a double line means that the rule holds in both directions). If $\mathrm{C}_{\varphi}=\operatorname{Con}(\mathrm{UL})$ then $\mathrm{UL}\left(\mathrm{C}_{\varphi}\right)$ is denoted UL. In these rules, $U$ and $V$ can be empty structures and in the diplay rules, for $i=0$, the structures $X, Y, Z$ can also be empty. If the structure $U$ is empty then $* U$ is also empty and $U,{ }_{0} X$ and $X,_{0} U$ both denote $X$. Moreover, in rule $\otimes_{K}^{\mathrm{i}}$ (for $i \in\{1,2,3\}$ ), the consequent of one of the premises can also be empty and in that case the consequent of the conclusion is also empty. For better readability, the brackets for binary connectives are omitted.

A parameter in an inference rule is a structure (or formula) which is either held constant from premises to conclusion or which is introduced with no regard to its particular (formulas introduced by weakening are also parameters). A principal formulas in an inference rule is a non-parametric formula occurring in the conclusion. Congruent parameters in an inference rule are parameters that occur both in the premise(s) and the conclusion of that inference rule and that correspond to the same formula/structure. In our sequent calculus UL of Figures 4 and 5 , principal formulas are represented by Greek formulas $\varphi, \psi$ and parameters are denoted by the Latin letters $X, Y, Z$. Congruent parameters are denoted by the same Latin letter (be it $X, Y$ or $Z)$.

The semantics of update logic was based on cyclic permutations, as explained after Definition 8 on Page 14. It turns out that these cyclic permutations reappear in the inference rules of our display calculus UL. Indeed, the rules for the substructural connectives and the display rules can be rewritten differently, like in Figure 6: $\{(1,2,3),(2,3,1),(3,1,2)\}=\{(i, \tau(i), \rho(i))$ | $i \in\{1,2,3\}\}$. Moreover, by our conventions of notation, the following rules are instances of the display rules:

$$
\frac{U \vdash V}{\overline{U, * V \vdash}} *_{A} \quad \frac{U, V \vdash}{\overline{U \vdash * V}} *_{K}
$$

Connection with Wansing's modal display calculus. If we consider $\mathbf{C}_{\varphi}:=\left\{\neg, \wedge, \square, \diamond^{-}\right\}$ then $\operatorname{UL}\left(\mathrm{C}_{\varphi}\right)$ is the display calculus for modal logic of Wansing [76] (restricted to these connectives and with a slight modification of the structural rules for negation $*$ ). Hence, our display calculus is a generalization of the display calculus for modal logic. However, unlike it, we do not resort in our calculus to the empty structure I. Usually, this empty structure is used to obtain the display property or to prove completeness of display calculi (by reduction to a Hilbert proof calculus [78]). Here, we do not need to use this empty structure I. Instead, we use more general rules for the structural connective $*$ and we resort in all cases to the classical structural connective,$_{0}$. These rules are more general than the rules of the modal display calculus of Wansing [76], because we can easily prove that every $\mathcal{S}\left(\mathbb{P}, \operatorname{Con}_{\varphi}\right)$-consecution is structurally equivalent 
Classical Rules:

$$
\frac{U \vdash V}{U, X \vdash V} \mathrm{~K} \quad \frac{X, X \vdash U}{X \vdash U} \mathrm{WI} \quad \frac{Y, X \vdash U}{X, Y \vdash U} \mathrm{Cl} \quad \frac{(X, Y), Z \vdash U}{X,(Y, Z) \vdash U} \mathrm{~B}^{c}
$$

Display Rules:

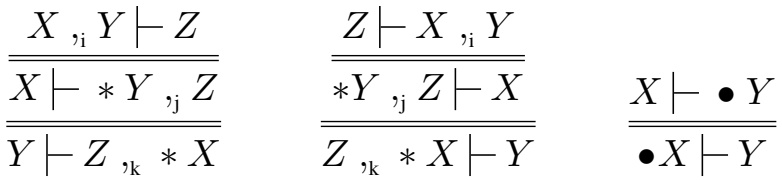

$$
\begin{aligned}
& (i, j, k) \in\{(0,0,0),(1,2,3),(2,3,1),(3,1,2)\}
\end{aligned}
$$

Cut Rule:

$$
\frac{U \vdash \varphi \quad \varphi \vdash V}{U \vdash V}
$$

Figure 4: Structural Rules

to a $\mathcal{S}\left(\mathbb{P}, \operatorname{Con}_{\varphi}\right)$-consecution where the antecedent is empty or the consequent is empty, like in linear logic [34]. Our approach simplifies somewhat the structural rules. Finally, restricted to the structural connective, (that is the structural connective,$_{0}$ ) our display rules are identical to the basic structural rules (1) and (2) of [76] (if we assume permutation $\mathrm{Cl}$ ).

Connection with Dunn's Gaggle Theory. We can relate our definitions with the terminology of Dunn's Gaggle Theory [24]. We will not recall this theory and we refer the reader to Dunn [24], or Goré [36] for a short but concise overview. If $C_{\varphi}:=\left\{T, \perp, \otimes_{i}, \supset_{j}, C_{k}\right\}$ for some $(i, j, k) \in\{(1,2,3),(2,3,1),(3,1,2)\}$, then the triple of connectives $O P_{i}:=\left\{\otimes_{\mathrm{i}}, \supset_{\mathrm{j}}, \subset_{\mathrm{k}}\right\}$ is a founded family of the partial gaggle $\mathcal{T}:=\left(\mathcal{L}\left(\mathbb{P}, \mathrm{C}_{\varphi}\right), \Vdash, O P_{i}\right)$ and $\otimes_{\mathrm{i}}$ is its head. In particular, each pair of connectives satisfy the abstract law of residuation and in that case we say that they are relative.

Usually, to construct a display calculus for a logic, we associate to each pair of residuated logical connectives a structural connectives [22]. This method has been successfully applied to modal logic for the case of unary modal connectives for which founded families often come in pairs [76, 78]. Somehow, we generalize this idea to the case of binary connectives. But instead of considering pairs of residuated binary connectives, we consider founded families of relative binary connectives which generally come in triples, such as $O P_{i}:=\left\{\otimes_{\mathrm{i}}, \supset_{\mathrm{j}}, \subset_{\mathrm{k}}\right\}$ for each $(i, j, k) \in\{(1,2,3),(2,3,1),(3,1,2)\}$. So, we associate to the head of each founded family a structural connective ${ }_{, i}$ and we use the structural connective $*$ associated to the Boolean 


\section{Axioms:}

$\overline{p \vdash p}^{I d} \quad \overline{\perp \vdash} \perp_{A} \quad \overline{\vdash \top}^{\top} K$

Propositional Connectives:

$\frac{U \vdash * \varphi}{U \vdash \neg \varphi} \neg K \quad \frac{* \varphi \vdash U}{\neg \varphi \vdash U} \neg A$

$\frac{U \vdash \varphi}{U \vdash \varphi \vee \psi} \vee_{K}^{1} \quad \frac{U \vdash \psi}{U \vdash \varphi \vee \psi} \vee_{K}^{2} \quad \frac{\varphi \vdash U \quad \psi \vdash U}{\varphi \vee \psi \vdash U} \vee_{A}$

$\frac{U \vdash \varphi \quad U \vdash \psi}{U \vdash \varphi \wedge \psi} \wedge_{K} \quad \frac{\varphi \vdash U}{\varphi \wedge \psi \vdash U} \wedge_{A}^{1} \quad \frac{\psi \vdash U}{\varphi \wedge \psi \vdash U} \wedge_{A}^{2}$

\section{Modal Connectives:}

$\frac{\bullet X \vdash \varphi}{X \vdash \square \varphi} \square_{K}$

$\frac{\varphi \vdash X}{\square \varphi \vdash \cdot X} \square_{A}$

$\frac{X \vdash \varphi}{\bullet X \vdash \diamond^{-} \varphi} \diamond_{K}^{-}$

$\frac{\varphi \vdash \cdot X}{\diamond^{-} \varphi \vdash X} \diamond_{A}^{-}$

Substructural Connectives:

$\frac{X \vdash \varphi \quad Y \vdash \psi}{X{ }_{, i} Y \vdash \varphi \otimes_{\mathrm{i}} \psi} \otimes_{K}^{\mathrm{i}} \quad \frac{\varphi,_{\mathrm{i}} \psi \vdash U}{\varphi \otimes_{\mathrm{i}} \psi \vdash U} \otimes_{A}^{\mathrm{i}}$

$\frac{X{ }_{,} \varphi \vdash \psi}{X \vdash \varphi \supset_{\mathrm{j}} \psi} \supset_{K}^{\mathrm{j}} \quad \frac{X \vdash \varphi \psi \vdash Y}{\varphi \supset_{\mathrm{j}} \psi \vdash * X{ }_{, \mathrm{j}} Y} \supset_{A}^{\mathrm{j}}$

$\frac{\varphi,{ }_{\mathrm{i}} X \vdash \psi}{X \vdash \psi \subset_{\mathrm{k}} \varphi} \subset_{K}^{\mathrm{k}} \quad \frac{\psi \vdash Y \quad X \vdash \varphi}{\psi \subset_{\mathrm{k}} \varphi \vdash Y{ }_{{ }_{\mathrm{k}}} * X} \subset_{A}^{\mathrm{k}}$

$(i, j, k) \in\{(1,2,3),(2,3,1),(3,1,2)\}$

Figure 5: Logical Rules for Propositional, Modal and Substructural Connectives 


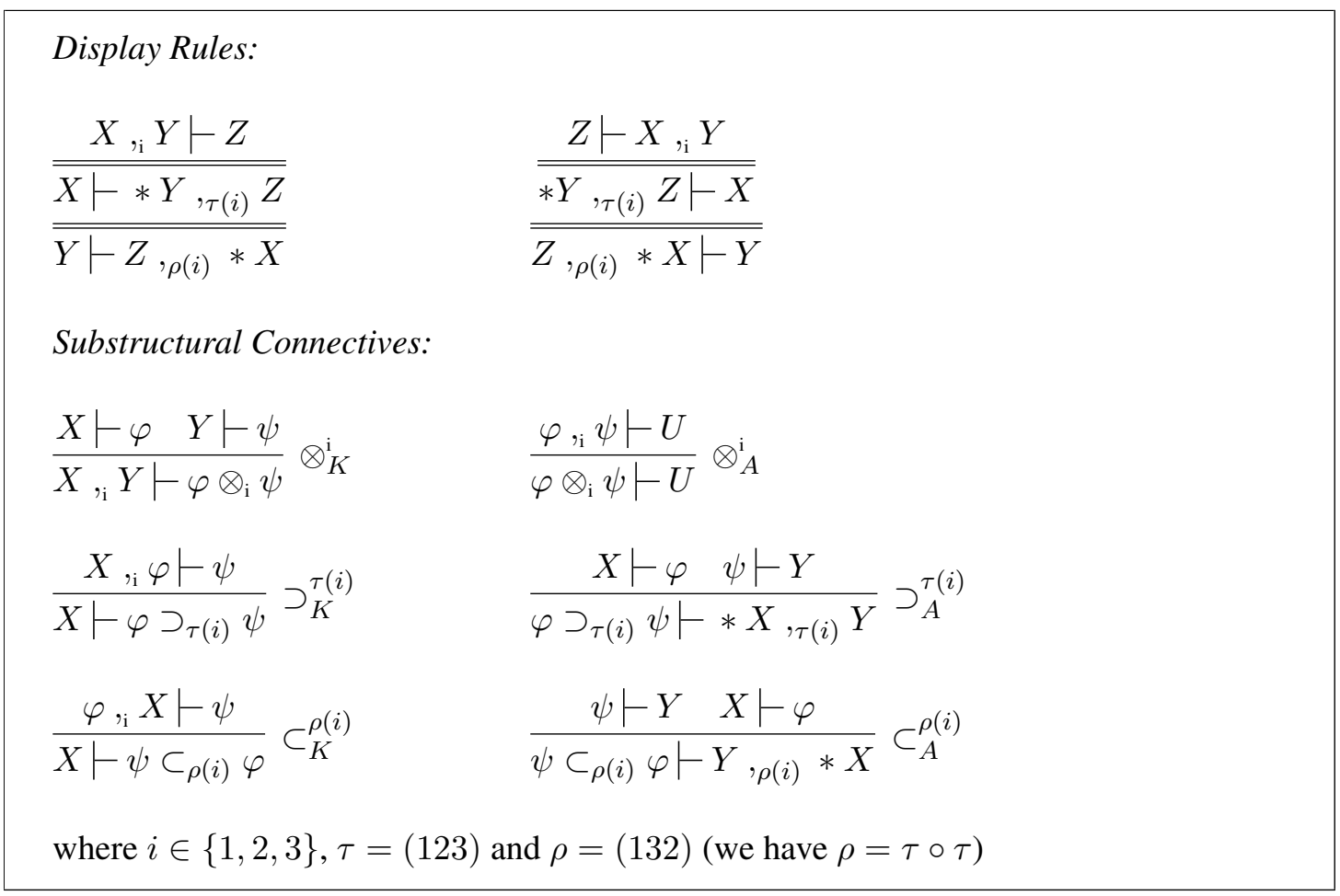

Figure 6: Inference Rules and Cyclic Permutations 


\begin{tabular}{|c|c|c|c|}
\hline Connective $f$ & Trace of $f$ & $S(f, \varphi, \psi)$ & Display rule \\
\hline $\begin{array}{l}\diamond^{-} \\
\square\end{array}$ & $\begin{array}{l}(-) \mapsto- \\
(+) \mapsto+\end{array}$ & $\begin{array}{c}\diamond^{-} \varphi \Vdash \psi \psi \\
\varphi \Vdash \square \psi\end{array}$ & $\begin{array}{l}\bullet X \| Y \\
X \| \bullet Y\end{array}$ \\
\hline Connective $f$ & Trace of $f$ & $S(f, \varphi, \chi, \psi)$ & Display rule \\
\hline $\begin{array}{l}\otimes_{\mathrm{i}} \\
\supset_{\mathrm{j}} \\
C_{\mathrm{k}}\end{array}$ & $\begin{array}{l}(-,-) \mapsto- \\
(-,+) \mapsto+ \\
(+,-) \mapsto++\end{array}$ & $\begin{array}{l}\varphi \otimes_{\mathrm{i}} \chi \| \psi \\
\varphi \| \chi \supset_{\mathrm{j}} \varphi \\
\chi \| \psi \subset_{\mathrm{k}} \varphi\end{array}$ & $\begin{array}{c}X,{ }_{,} Y \| Z \\
X \Vdash * Y{ }_{, j} Z \\
Y \| Z,_{, k} * X\end{array}$ \\
\hline
\end{tabular}

$$
(i, j, k) \in\{(1,2,3),(2,3,1),(3,1,2)\}
$$

Figure 7: Transforming Founded Families into Display Rules

negation to define the display rules associated to this founded family of connectives. In fact, there is a very close similarity between, on the one hand, the expressions $S(f, \varphi, \chi, \psi)$ (for $f \in$ $O P_{i}$ ) obtained from the traces of $f$ and, on the other hand, the consecutions of our display rules ${ }^{4}$ One can even obtain our display rules from these expressions by the successive transformations given in Figure 7 (from left to right). The third set of display rules will be obtained when we will consider dual substructural connectives in Section 7.2 .

Connection with Goré's display logic. There is also a close similarity between our display rules and the structural rules introduced by Goré [36] associated to his structural connectives $\circ,<,>$. We reproduce them below:

$$
\begin{array}{ll}
\frac{Y \vdash X>Z}{X \circ Y \vdash Z} & \frac{X>Z \vdash Y}{Z \vdash X \circ Y} \\
\hline X \vdash Z<Y & \frac{Z \vdash Y \vdash X}{Z<Y \vdash X}
\end{array}
$$

With a closer inspection, the structural connective $X \circ Y$ corresponds to our connective $X,{ }_{3} Y$, the structural connective $X>Z$ is an abbreviation of our $Z,_{2} * X$ and the structural connective $Z<Y$ is an abbreviation of our $* Y,{ }_{1} Z$. Moreover, Goré [36] also deals with negation and he obtains display rules for negation (called (Grn DP $\vdash$ ) and $(\vdash$ Grn DP)) that are again closely related to our display rules.

Theorem 2 (Soundness and strong completeness). The display calculus UL is sound and strongly complete for the logic $\left(\mathcal{C}\left(\operatorname{Con}_{\varphi}\right), \mathcal{E}, \Vdash\right)$.

Proof. See Section 5 .

\footnotetext{
${ }^{4}$ See for instance [36, 78] for a definition of the trace of a connective $f$ and an explanation of the notation $S(f, \varphi, \chi, \psi)$.
} 


\subsection{Admissibility of the Cut Rule}

Theorem 3 below shows that UL is a display calculus: each antecedent (consequent) part of a consecution can be 'displayed' as the sole antecedent (resp. consequent) of a structurally equivalent consecution.

Definition 14 (Antecedent and consequent part). Let $X$ be a $\mathcal{S}\left(\mathbb{P}\right.$, Con $\left._{\varphi}\right)$-structure and let $Y$ be a substructure of $X$. We say that $Y$ occurs positively in $X$ if it is in the scope of an even number of $*$. Otherwise, if $Y$ is in the scope of an odd number of $*$ in $X$, we say that $Y$ occurs negatively in $X$. If $X \vdash Y$ is a $\mathcal{S}\left(\mathbb{P}, \operatorname{Con}_{\varphi}\right)$-consecution, then $X$ is called the antecedent and $Y$ is called the consequent. Let $Z$ be a substructure of $X$ or $Y$. We say that $Z$ is an antecedent part of $X \vdash Y$ if $Z$ occurs positively in $X$ or negatively in $Y$. We say that $Z$ is a consequent part of $X \vdash Y$ if $Z$ occurs positively in $Y$ or negatively in $X$.

Theorem 3 (Display Theorem for UL). For each $\mathcal{S}\left(\mathbb{P}\right.$, Con $\left._{\varphi}\right)$-consecution $X \vdash Y$ and each antecedent part (respectively consequent part) $Z$ of $X \vdash Y$, if $X \vdash Y$ is provable in $U L$ then there exists a $\mathcal{L}\left(\mathbb{P}, \operatorname{Con}_{\varphi}\right.$, Con $\left._{X}\right)$-structure $W$ such that $Z \vdash W$ (respectively $\left.W \vdash Z\right)$ is provable in UL.

Proof. The proof follows the same lines as the proof of [78, Theorem 12]. The case when $f=* g$ is proved by using the third derived rule of Expression 5 .

In the Expressions (5), we show how the classical structural rules of [78] for negation can be recovered in our display calculus UL ( $\mathrm{dr}_{2}$ denotes an application of one of the second set of display rules).

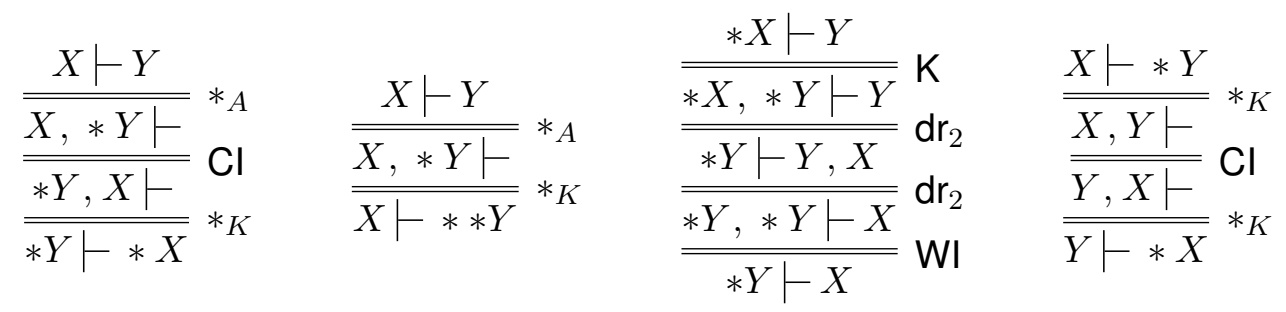

Note that in the above proofs (especially the third proof), we use the structural rules for the classical structural connective $*$ and,$_{0}$. This explains why we impose that $\left\{*,,_{0}\right\} \subseteq \operatorname{Struc}\left(\mathrm{C}_{\varphi}\right)$ for all logical connectives $\mathrm{C}_{\varphi} \subseteq \operatorname{Con}_{\varphi}$. We recall that the semantics of these structural connectives is classical: * corresponds to the Boolean negation and ${ }_{2}$ corresponds to conjunction or disjunction, depending on the context (whether it is in antecedent or consequent position). So, when we try to prove a statement using our display calculus, we manipulate structures and substructures in consecutions at the structural level, that is, some sort of 'meta' level at which we always reason classically. As a matter of fact, this is also what we do when we prove a valid statement semantically, using the truth conditions of the logic. These truth conditions are expressed in natural language at the 'meta' level but comply to the reasoning of classical logic. So, in a sense, the structural reasoning in UL could be viewed as the classical meta-reasoning used to prove a theorem/validity of update logic. 
Now, we show that UL is in fact a proper display calculus (see [17, 78] for more detailed explanations of the conditions $(C 1)-(C 8)$ listed below).

Definition 15 (Proper display calculus). A sequent calculus is a proper display calculus when each of its inference rules satisfies the following eight conditions $(C 1)-(C 8)$ :

(C1) Preservation of formulas. Each formula occurring in a premise of a rule is the subformula of some formula in the conclusion of that rule.

(C2) Shape-alikeness of parameters. Congruent parameters in a rule are occurrences of the same structures.

(C3) Non-proliferation of parameters. Each parameter of any rule is congruent to at most one parameter in the conclusion of that rule.

(C4) Position-alikeness of parameters. Congruent parameters are either all antecedent or all consequent parts of their respective consecutions.

(C5) Display of principal constituents. A principal formula of any rule is either the entire antecedent or the entire consequent of the conclusion of this rule.

(C6) Closure under substitution for consequent parts. Each rule is closed under simultaneous substitution of arbitrary structures for congruent formulas which are consequent parts.

(C7) Closure under substitution for antecedent parts. Each rule is closed under simultaneous substitution of arbitrary structures for congruent formulas which are antecedent parts.

(C8) Eliminability of matching principal formulas. If there are inferences $\inf _{1}$ and $\inf _{2}$ with respective conclusions (1) $X \vdash \varphi$ and (2) $\varphi \vdash Y$ with $\varphi$ principal in both inferences, and if cut is applied to obtain (3) $X \vdash Y$, then either (3) is identical to (1) or (2), or there is a proof of (3) from the premises of $\inf _{1}$ and $\inf _{2}$ in which every cut-formula of any application of cut is a proper formula of $\varphi$.

Theorem 4 (Strong cut elimination). The display calculus UL is a proper display calculus. Hence, UL enjoys strong cut-elimination and therefore the cut rule is an admissible rule of UL.

Proof. The logical rules were already introduced and studied in [78], so we only need to check the structural rules. A rapid inspection shows that they indeed respect the eight conditions. Therefore, UL is a proper display calculus. It enjoys strong cut-elimination because of [78, Theorem 23].

Corollary 1. Let $C_{\varphi} \subseteq \operatorname{Con}_{\varphi}$. The display calculus $\operatorname{UL}\left(C_{\varphi}\right)$ is sound and strongly complete for the logic $\left(\mathcal{C}\left(C_{\varphi}\right), \mathcal{E}, \Vdash\right)$.

Proof. The soundness comes from Theorem 2. For strong completeness, consider $\Gamma \subseteq \mathcal{C}\left(\mathrm{C}_{\varphi}\right)$ and $S \in \mathcal{C}\left(\mathrm{C}_{\varphi}\right)$. If $\Gamma \Vdash S$ holds then $\Gamma \vdash S$ is provable in UL by Theorem 2 However, because UL enjoys strong cut elimination (Theorem 4), a proof of $S$ from $\Gamma$ is a proof that contains only the subformulas contained in $S$ or $\Gamma$, and therefore a proof that contains only the connectives of $\mathrm{C}_{\varphi}$. That is, $\Gamma \vdash S$ is provable in $\mathrm{UL}\left(\mathrm{C}_{\varphi}\right)$. 


\section{Completeness Proof of the Display Calculus for Update Logic}

In this section we prove Theorem 2. The proof of soundness is without particular difficulty. The completeness proof relies on a new method that is not specific to our update logic but applies to any Gentzen and display-style calculi. It is also related to the completeness proof method of Wansing [77, Section 10.6] for the display calculus of modal (tense) logic. Henkin constructions for Lambek calculi already exist. On the one hand, they are somewhat simpler than our approach. The set of worlds of the canonical model in these constructions is simply the set of all formulas of the language, and we set $\mathcal{R} x y z$ when $z \vdash x \otimes y$ is derivable, and $x \models p$ when $x \vdash p$ is also derivable. On the other hand, our approach is closer to standard techniques of modal logic and it will allow us to import the existing correspondence results for substructural logics.

\subsection{Preliminary Definitions and Results}

First, we define the notions of UL-consistent set and maximal UL-consistent set. In the sequel, by abuse of notation and to ease the presentation, when we write $X \vdash Y$ we mean that $X \vdash Y$ is provable in UL.

Definition 16 ((Maximal) UL-consistent set).

- A $U L$-consistent set is a subset $\Gamma$ of $\mathcal{S}\left(\mathbb{P}, \operatorname{Con}_{\varphi}\right)$ such that there are no $X_{1}, \ldots, X_{n} \in \Gamma$ such that $X_{1}, \ldots, X_{n} \vdash$.

- A maximal $U L$-consistent set is a UL-consistent subset $\Gamma$ of $\mathcal{S}\left(\mathbb{P}, \operatorname{Con}_{\varphi}\right)$ such that there is no $X \in \mathcal{S}\left(\mathbb{P}, \operatorname{Con}_{\varphi}\right)$ such that $X \notin \Gamma$ and $\Gamma \cup\{X\}$ is UL-consistent.

Lemma 5 (Cut Lemma). Let $\Gamma$ be a maximal UL-consistent set. For all $X_{1}, \ldots, X_{k} \in \Gamma$, all $\varphi \in \mathcal{L}\left(\mathbb{P}, \operatorname{Con}_{\varphi}\right)$, if $X_{1}, \ldots, X_{k} \vdash \varphi$ then $\varphi \in \Gamma$.

Proof. First, we show that $\Gamma \cup\{\varphi\}$ is $U L$-consistent. Assume towards a contradiction that it is not the case. Then, there is $Y_{1}, \ldots, Y_{l} \in \Gamma$ such that $Y_{1}, \ldots, Y_{l}, \varphi \vdash$. So, by the basic structural rules, we have that $\varphi \vdash *\left(Y_{1}, \ldots, Y_{l}\right)$. Therefore, by the cut rule, we have that $X_{1}, \ldots, X_{k} \vdash *\left(Y_{1}, \ldots, Y_{l}\right)$. Then, again by the basic structural rules, we have that $\left(Y_{1}, \ldots, Y_{l}\right),\left(X_{1}, \ldots, X_{k}\right) \vdash$, that is $Y_{1}, \ldots, Y_{l}, X_{1}, \ldots, X_{k} \vdash$ by associativity. Because $X_{1}, \ldots, X_{k}, Y_{1}, \ldots, Y_{l} \in \Gamma$, we conclude that $\Gamma$ is not UL-consistent, which is impossible. Thus, our initial assumption was wrong and $\Gamma \cup\{\varphi\}$ is UL-consistent. Then, because $\Gamma$ is a maximal UL-consistent set, we have finally that $\varphi \in \Gamma$.

Then, we have the following Lindenbaum-like Lemma.

Lemma 6 (Lindenbaum Lemma). Any UL-consistent set can be extended into a maximal ULconsistent set.

Proof. Let $\Gamma$ be a UL-consistent set and let $X_{0}, X_{1}, \ldots, X_{n}, \ldots$ be an enumeration of $\mathcal{S}\left(\mathbb{P}, \operatorname{Con}_{\varphi}, \operatorname{Con}_{X}\right)$ (it exists because $\mathbb{P}$ is countable). We define inductively the sets $\Gamma_{n}$ as 
follows:

$$
\begin{aligned}
\Gamma_{0} & :=\Gamma \\
\Gamma_{n+1} & := \begin{cases}\Gamma_{n} \cup\left\{X_{n}\right\} & \text { if } \Gamma_{n} \cup\left\{X_{n}\right\} \text { is UL-consistent; } \\
\Gamma_{n} & \text { otherwise }\end{cases}
\end{aligned}
$$

Then, we define the subset $\Gamma^{+}$of $\mathcal{S}\left(\mathbb{P}, \operatorname{Con}_{\varphi}, \operatorname{Con}_{X}\right)$ as follows: $\Gamma^{+}:=\bigcup_{n \geq 0} \Gamma_{n}$.

We show that $\Gamma^{+}$is a maximal UL-consistent set. Clearly, for all $n \in \mathbb{N}, \Gamma_{n}$ is UL-consistent by definition of $\Gamma_{n}$. So, if $\Gamma^{+}$was not UL-consistent, there would be $n_{0} \in \mathbb{N}$ such that $\Gamma_{n_{0}}$ is not UL-consistent, which is impossible. Now, assume towards a contradiction that $\Gamma^{+}$is not a maximal UL-consistent set. Then, there is $X \in \mathcal{S}\left(\mathbb{P}, \operatorname{Con}_{\varphi}, \operatorname{Con}_{X}\right)$ such that $X \notin \Gamma^{+}$and $\Gamma^{+} \cup\{X\}$ is UL-consistent. But there is $n \in \mathbb{N}$ such that $X=X_{n}$. Because $X \notin \Gamma^{+}$, we also have that $X_{n} \notin \Gamma_{n}$. So, $\Gamma_{n} \cup\left\{X_{n}\right\}$ is not UL-consistent by definition of $\Gamma^{+}$. Therefore, $\Gamma^{+} \cup\{X\}$ is not $U L$-consistent, which is impossible.

Finally, we prove some facts which will be used in the completeness proof, more precisely in the proof of the (truth) Lemma 10

Lemma 7. For all $\varphi \in \mathcal{L}\left(\mathbb{P}\right.$, Con $\left._{\varphi}\right)$, all $i \in\{1,2,3\}$ and all $X \vdash Y \in \mathcal{C}\left(\operatorname{Con}_{\varphi}\right)$,

$$
\begin{gathered}
\varphi \vdash \varphi \\
X \vdash \tau_{1}(X) \\
\tau_{2}(X) \vdash X \\
X \vdash Y \quad \text { iff } \tau_{1}(X) \vdash \tau_{2}(Y) \\
\quad \text { iff } X \vdash \tau_{2}(Y) \\
\quad \text { iff } \tau_{1}(X) \vdash Y \\
\varphi \otimes_{i}(\chi \vee \rho) \vdash\left(\varphi \otimes_{i} \chi\right) \vee\left(\varphi \otimes_{i} \rho\right) \\
\psi \subset_{i} \varphi_{1}, \ldots, \psi \subset_{i} \varphi_{n} \vdash \psi \subset_{i}\left(\varphi_{1} \vee \ldots \vee \varphi_{n}\right)
\end{gathered}
$$

(The mappings $\tau_{1}$ and $\tau_{2}$ are defined in Proposition 1)

Proof. Expressions (6), (7), (8) and (9) are proved by an easy induction on $\varphi, X$ and $Y$. The proof of Expression (10) is as follows ( $\mathrm{dr}_{1}$ denotes an application of the first set of display rules): 


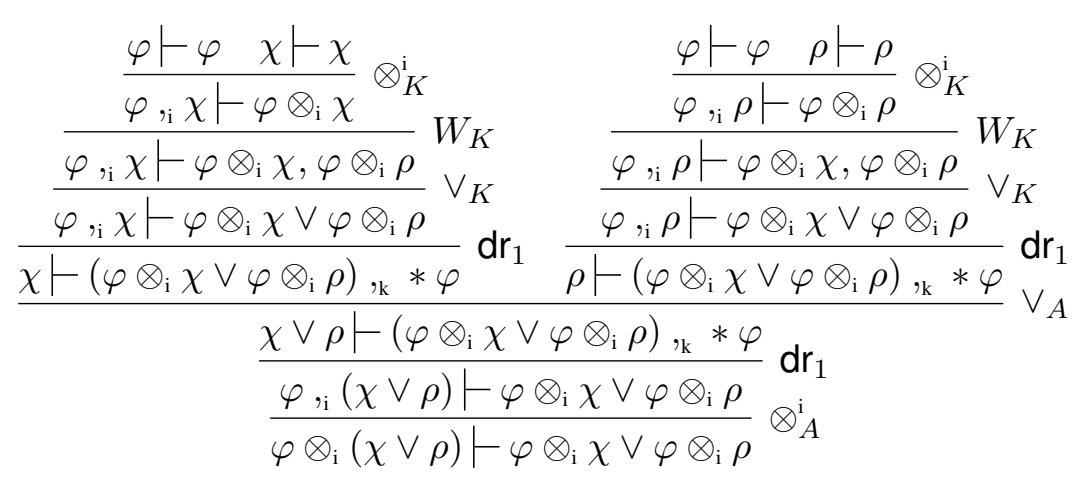

The proof of Expression (11) is as follows. By iterated application of $\vee_{A}$ and the structural rules to the consecutions $\varphi_{1} \vdash \varphi_{1}, \ldots, \varphi_{n-1} \vdash \varphi_{n-1}$ and $\varphi_{n} \vdash \varphi_{n}$, we obtain that $\varphi_{1} \vee \ldots \vee$ $\varphi_{n} \vdash \varphi_{1}, \ldots, \varphi_{n}$. Then, by application of $\subset_{A}^{\mathrm{i}}$ and $\subset_{K}^{\mathrm{i}}$ to $\varphi_{1} \vee \ldots \vee \varphi_{n} \vdash \varphi_{1}, \ldots, \varphi_{n}$ and $\psi \vdash \psi$, we have that $\left(\varphi_{1} \vee \ldots \vee \varphi_{n}\right)_{3}\left(\psi \subset_{2} \varphi_{1}, \ldots, \psi \subset_{2} \varphi_{n}\right) \vdash \psi$. Finally, by $\subset_{K}^{2}$, we obtain that $\psi \subset_{\mathrm{i}} \varphi_{1}, \ldots, \psi \subset_{\mathrm{i}} \varphi_{n} \vdash \psi \subset_{\mathrm{i}}\left(\varphi_{1} \vee \ldots \vee \varphi_{n}\right)$.

Lemma 8. Let $\varphi, \chi \in \mathcal{L}\left(\mathbb{P}\right.$, Con $\left._{\varphi}\right)$. If $\varphi \otimes_{3} \chi$ is UL-consistent, then $\varphi$ and $\chi$ are UL-consistent.

Proof. We only prove that $\varphi$ is UL-consistent, the proof for $\chi$ being similar. Assume towards a contradiction that $\varphi$ is not UL-consistent, that is $\varphi \vdash$. Then, because $\chi \vdash \chi$ by Expression 6 of Lemma 7, we have by application of $\otimes_{K}^{3}$ and then $\otimes_{A}^{3}$ that $\varphi \otimes_{3} \chi \vdash$. Hence, $\varphi \otimes_{3} \chi$ is not UL-consistent, which is impossible.

\subsection{The Completeness Proof}

We define the canonical substructural model associated to UL.

Definition 17. We define the point set $\mathcal{P}^{c}$, the binary relations $\left\{\mathcal{R}_{\square}^{c}, \mathcal{R}_{\diamond^{-}}^{c}\right\}$ and the ternary relations $\left\{\mathcal{R}_{\otimes_{\mathrm{i}}}^{c}, \mathcal{R}_{\supset_{\mathrm{i}}}^{c}, \mathcal{R}_{\subset_{\mathrm{i}}}^{c} \mid i \in\{1,2,3\}\right\}$ as follows:

- $\mathcal{P}^{c}:=\left(P^{c},=\right)$ where $P^{c}$ is the set of all maximal UL-consistent sets; 
- for all $\Gamma, \Gamma^{\prime}, \Gamma^{\prime \prime} \in P^{c}$,

\begin{tabular}{|c|c|c|}
\hline $\begin{array}{l}\left(\Gamma, \Gamma^{\prime}\right) \in \mathcal{R}_{\square}^{c} \\
\left(\Gamma, \Gamma^{\prime}\right) \in \mathcal{R}_{\diamond}^{c}\end{array}$ & $\begin{array}{l}\text { iff } \\
\text { iff }\end{array}$ & $\begin{array}{l}\text { for all } \square \varphi \in \Gamma \text {, we have that } \varphi \in \Gamma^{\prime} \\
\text { for all } \varphi \in \Gamma^{\prime}, \text { we have that } \diamond^{-} \varphi \in \Gamma\end{array}$ \\
\hline $\begin{array}{l}\left(\Gamma, \Gamma^{\prime}, \Gamma^{\prime \prime}\right) \in \mathcal{R}_{\supset_{1}}^{c} \\
\left(\Gamma, \Gamma^{\prime}, \Gamma^{\prime \prime}\right) \in \mathcal{R}_{\triangle_{2}}^{c} \\
\left(\Gamma, \Gamma^{\prime}, \Gamma^{\prime \prime}\right) \in \mathcal{R}_{\otimes_{3}}^{c_{2}}\end{array}$ & $\begin{array}{l}\text { iff } \\
\text { iff } \\
\text { iff }\end{array}$ & $\begin{array}{l}\text { for all } \chi \supset_{1} \varphi \in \Gamma, \text { if } \chi \in \Gamma^{\prime} \text { then } \varphi \in \Gamma^{\prime \prime} \\
\text { for all } \psi \subset_{2} \varphi \in \Gamma^{\prime}, \text { if } \varphi \in \Gamma \text { then } \psi \in \Gamma^{\prime \prime} \\
\text { for all } \varphi \in \Gamma \text { and all } \chi \in \Gamma^{\prime}, \varphi \otimes_{3} \chi \in \Gamma^{\prime \prime}\end{array}$ \\
\hline $\begin{array}{l}\left(\Gamma, \Gamma^{\prime}, \Gamma^{\prime \prime}\right) \in \mathcal{R}_{\supset_{2}}^{c} \\
\left(\Gamma, \Gamma^{\prime}, \Gamma^{\prime \prime}\right) \in \mathcal{R}_{\subset_{3}}^{c^{\prime}} \\
\left(\Gamma, \Gamma^{\prime}, \Gamma^{\prime \prime}\right) \in \mathcal{R}_{\otimes_{1}}^{c^{2}}\end{array}$ & $\begin{array}{l}\text { iff } \\
\text { iff } \\
\text { iff }\end{array}$ & $\begin{array}{l}\text { for all } \psi \supset_{2} \varphi \in \Gamma^{\prime} \text {, if } \psi \in \Gamma^{\prime \prime} \text { then } \varphi \in \Gamma \\
\text { for all } \varphi \subset_{3} \chi \in \Gamma^{\prime \prime}, \text { if } \chi \in \Gamma^{\prime} \text { then } \varphi \in \Gamma \\
\text { for all } \psi \in \Gamma^{\prime \prime} \text { and all } \chi \in \Gamma^{\prime}, \chi \otimes_{1} \psi \in \Gamma\end{array}$ \\
\hline $\begin{array}{l}\left(\Gamma, \Gamma^{\prime}, \Gamma^{\prime \prime}\right) \in \mathcal{R}_{\supset_{3}}^{c} \\
\left(\Gamma, \Gamma^{\prime}, \Gamma^{\prime \prime}\right) \in \mathcal{R}_{\subset_{1}}^{c_{1}} \\
\left(\Gamma, \Gamma^{\prime}, \Gamma^{\prime \prime}\right) \in \mathcal{R}_{\otimes_{2}}^{c^{2}}\end{array}$ & $\begin{array}{l}\text { iff } \\
\text { iff }\end{array}$ & $\begin{array}{l}\text { for all } \varphi \supset_{3} \chi \in \Gamma^{\prime \prime}, \text { if } \varphi \in \Gamma \text { then } \chi \in \Gamma^{\prime} \\
\text { for all } \chi \subset_{1} \varphi \in \Gamma^{\prime \prime}, \text { if } \varphi \in \Gamma^{\prime \prime} \text { then } \chi \in \Gamma^{\prime} \\
\text { for all } \psi \in \Gamma^{\prime \prime} \text { and all } \varphi \in \Gamma, \psi \otimes_{2} \varphi \in \Gamma^{\prime} .\end{array}$ \\
\hline
\end{tabular}

Lemma 9. The following hold:

- $\mathcal{R}_{\square}^{c}=\mathcal{R}_{\diamond-}^{c}$;

- $\mathcal{R}_{\otimes_{1}}^{c}=\mathcal{R}_{\otimes_{2}}^{c}=\mathcal{R}_{\otimes_{3}}^{c}=\mathcal{R}_{\subset_{1}}^{c}=\mathcal{R}_{\subset_{2}}^{c}=\mathcal{R}_{\subset_{3}}^{c}=\mathcal{R}_{\supset_{1}}^{c}=\mathcal{R}_{\supset_{2}}^{c}=\mathcal{R}_{\supset_{3}}^{c}$.

Proof. We prove each item separately.

- For the first item, we prove that $\mathcal{R}_{\square}^{c} \subseteq \mathcal{R}_{\diamond-}^{c}$ and $\mathcal{R}_{\diamond-}^{c} \subseteq \mathcal{R}_{\square}^{c}$. Assume that $\left(\Gamma, \Gamma^{\prime}\right) \in \mathcal{R}_{\square}^{c}$. We are going to prove that $\left(\Gamma, \Gamma^{\prime}\right) \in \mathcal{R}_{\diamond}^{c}$. Let $\varphi \in \Gamma$. Then $\square \diamond^{-} \varphi \in \Gamma$, because $\varphi \vdash \square \diamond^{-} \varphi$ and application of Lemma 5 . So, by definition of $\mathcal{R}_{\square}^{c}$, we have that $\diamond^{-} \varphi \in \Gamma^{\prime}$. This holds for all $\varphi \in \Gamma$. Thus, $\left(\Gamma, \Gamma^{\prime}\right) \in \mathcal{R}_{\diamond-}^{c}$. The proof that $\mathcal{R}_{\diamond-}^{c} \subseteq \mathcal{R}_{\square}^{c}$ is similar and uses the fact that $\varphi \vdash \square^{-} \diamond \varphi$.

- For the second item, the proofs that $\mathcal{R}_{\supset_{1}}^{c}=\mathcal{R}_{\subset_{2}}^{c}=\mathcal{R}_{\otimes_{3}}^{c}$, that $\mathcal{R}_{\subset_{3}}^{c}=\mathcal{R}_{\supset_{2}}^{c}=\mathcal{R}_{\otimes_{1}}^{c}$ and that $\mathcal{R}_{\supset_{3}}^{c}=\mathcal{R}_{\subset_{1}}^{c}=\mathcal{R}_{\otimes_{2}}^{c}$ are similar to the proof of Restall [61, Lemma 11.25]. So, we prove that $\mathcal{R}_{\otimes_{3}}^{c}=\mathcal{R}_{\otimes_{1}}^{c}=\mathcal{R}_{\otimes_{2}}^{c}$. In fact, we only prove $\mathcal{R}_{\otimes_{3}}^{c}=\mathcal{R}_{\otimes_{1}}^{c}$, the proof that $\mathcal{R}_{\otimes_{1}}^{c}=\mathcal{R}_{\otimes_{2}}^{c}$ is similar. We start to prove that $\mathcal{R}_{\otimes_{3}}^{c} \subseteq \mathcal{R}_{\otimes_{1}}^{c}$. Let $\left(\Gamma, \Gamma^{\prime}, \Gamma^{\prime \prime}\right) \in \mathcal{R}_{\otimes_{3}}^{c}$, we are going to show that $\left(\Gamma, \Gamma^{\prime}, \Gamma^{\prime \prime}\right) \in \mathcal{R}_{\otimes_{1}}^{c}$. Let $\psi \in \Gamma^{\prime \prime}$ and $\chi \in \Gamma^{\prime}$. We are going to prove that $\chi,_{1} \psi \in \Gamma$. Assume towards a contradiction that $\chi{ }_{1} \psi \notin \Gamma$. Then, there are $X_{1}, \ldots, X_{n} \in \Gamma$ such that $\left(X_{1}, \ldots, X_{n}\right),\left(\chi,{ }_{1} \psi\right) \vdash$, because $\Gamma$ is a maximal consistent set. Then, we have the following derivation, using the display rules $\left(\mathrm{dr}_{1}\right.$ and $\mathrm{dr}_{3}$ denote an application of one of the first and third set of display rules respectively):

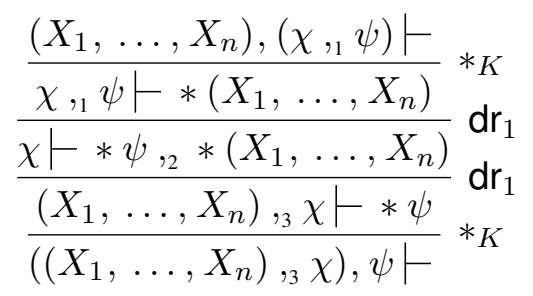


However, $\left(X_{1}, \ldots, X_{n}\right){ }_{{ }_{3}} \chi \in \Gamma^{\prime \prime}$ by Lemma 10 and definition of $\mathcal{R}_{\otimes_{3}}^{c}$, and $\psi \in \Gamma^{\prime \prime}$ by assumption. So, $\Gamma^{\prime \prime}$ is not UL-consistent, which is impossible. Hence, $\mathcal{R}_{\otimes_{3}}^{c} \subseteq \mathcal{R}_{\otimes_{1}}^{c}$. The proof of the converse, namely $\mathcal{R}_{\otimes_{3}}^{c} \supseteq \mathcal{R}_{\otimes_{1}}^{c}$, is symetrical; only the indices need to be changed.

From now on, $\mathcal{R}^{c}$ is any relation of $\left\{\mathcal{R}_{\otimes_{\mathrm{i}}}^{c}, \mathcal{R}_{\supset_{\mathrm{i}}}^{c}, \mathcal{R}_{\subset_{\mathrm{i}}}^{c} \mid i \in\{1,2,3\}\right\}$ and $R^{c}:=\mathcal{R}_{\square}^{c}$.

Definition 18 (Canonical model associated to UL). The canonical substructural model associated to $U L$ is the substructural model $\mathcal{M}^{c}:=\left(\mathcal{P}^{c}, R^{c}, \mathcal{R}^{c}, \mathcal{I}^{c}\right)$ where for all $p \in \mathbb{P}$, we have $p \in \mathcal{I}^{c}(\Gamma)$ if, and only if, $p \in \Gamma$.

Lemma 10 (Truth Lemma). For all $\varphi \in \mathcal{L}\left(\mathbb{P}\right.$, Con $\left._{\varphi}\right)$, for all maximal UL-consistent sets $\Gamma$, we have that

$$
\mathcal{M}^{c}, \Gamma \| \varphi \text { iff } \varphi \in \Gamma \text {. }
$$

Proof. The proof is by induction on the formula $\varphi$. The base case $\varphi:=p$ holds trivially by definition of $\mathcal{I}^{c}$. Now, we prove the induction steps:

$\bullet \neg \psi$ :

Assume that $\neg \psi \in \Gamma$. If $\psi \in \Gamma$, then because $\psi, \neg \psi \vdash$ and $\psi, \neg \psi \in \Gamma, \Gamma$ would not be UL-consistent. Therefore, it is not the case that $\psi \in \Gamma$, that is, by Induction Hypothesis, it is not the case that $\mathcal{M}^{c}, \Gamma \Vdash \psi$. That is, $\mathcal{M}^{c}, \Gamma \Vdash \neg \psi$.

For the other direction, if $\mathcal{M}^{c}, \Gamma \Vdash \neg \psi$, then it is not the case that $\mathcal{M}^{c}, \Gamma \Vdash \psi$. So, by Induction Hypothesis, $\psi \notin \Gamma$. Because $\Gamma$ is a maximal UL-consistent set, there are $X_{1}, \ldots, X_{n} \in \Gamma$ such that $X_{1}, \ldots, X_{n}, \psi \vdash$. Therefore, by the display rules and the rule $\neg_{K}$, we have that $X_{1}, \ldots, X_{n} \vdash \neg \psi$. Hence, by Lemma 5, we have that $\neg \psi \in \Gamma$.

- $\varphi_{1} \wedge \varphi_{2}$ :

$\mathcal{M}^{c}, \Gamma \models \varphi_{1} \wedge \varphi_{2}$ iff $\mathcal{M}^{c}, \Gamma \models \varphi_{1}$ and $\mathcal{M}^{c}, \Gamma \models \varphi_{2}$ by definition, iff $\varphi_{1} \in \Gamma$ and $\varphi_{2} \in \Gamma$ by Induction Hypothesis. Now, we prove that $\varphi_{1} \in \Gamma$ and $\varphi_{2} \in \Gamma$ iff $\varphi_{1} \wedge \varphi_{2} \in \Gamma$. That will prove this induction step. First, we prove that if $\varphi_{1} \in \Gamma$ and $\varphi_{2} \in \Gamma$ then $\varphi_{1} \wedge \varphi_{2} \in \Gamma$. By Expression (6), we have that $\varphi_{1} \vdash \varphi_{1}$ and $\varphi_{2} \vdash \varphi_{2}$. So, by the rule $\wedge_{K}$, we have that $\varphi_{1}, \varphi_{2} \vdash \varphi_{1} \wedge \varphi_{2}$. But $\varphi_{1}, \varphi_{2} \in \Gamma$. Therefore, by Lemma 5, we have that $\varphi_{1} \wedge \varphi_{2} \in \Gamma$. Second, we prove that $\varphi_{1} \wedge \varphi_{2} \in \Gamma$ entails that $\varphi_{1} \in \Gamma$ and $\varphi_{2} \in \Gamma$. Assume that $\varphi_{1} \wedge \varphi_{2} \in \Gamma$ and assume towards a contradiction that $\varphi_{1} \notin \Gamma$ (the proof for the case $\varphi_{2} \notin \Gamma$ is similar). Then, there are $X_{1}, \ldots, X_{n} \in \mathcal{L}$ such that $X_{1}, \ldots, X_{n}, \varphi_{1} \vdash$. So, by the display rules, we have that $\varphi_{1} \vdash *\left(X_{1}, \ldots, X_{n}\right)$. Therefore, by rule $\wedge_{A}^{1}$, we also have that $\varphi_{1} \wedge \varphi_{2} \vdash *\left(X_{1}, \ldots, X_{n}\right)$ and by the display rules we obtain that $X_{1}, \ldots, X_{n}, \varphi_{1} \wedge \varphi_{2} \vdash$. However, $X_{1}, \ldots, X_{n}, \varphi_{1} \wedge \varphi_{2} \in \Gamma$, so it entails that $\Gamma$ is not UL-consistent by definition of consistency, which is impossible. Therefore, $\varphi_{1} \in \Gamma$. We prove similarly that $\varphi_{2} \in \Gamma$. 
- $\varphi_{1} \vee \varphi_{2}$

$\mathcal{M}^{c}, \Gamma \models \varphi_{1} \vee \varphi_{2}$ iff $\mathcal{M}^{c}, \Gamma \models \varphi_{1}$ or $\mathcal{M}^{c}, \Gamma \models \varphi_{2}$ by definition, iff $\varphi_{1} \in \Gamma$ or $\varphi_{2} \in \Gamma$ by Induction Hypothesis. Now, we prove that $\varphi_{1} \in \Gamma$ or $\varphi_{2} \in \Gamma$ iff $\varphi_{1} \vee \varphi_{2} \in \Gamma$. That will prove this induction step. First, we prove that if $\varphi_{1} \in \Gamma$ or $\varphi_{2} \in \Gamma$ then $\varphi_{1} \vee \varphi_{2} \in \Gamma$. Without loss of generality, assume that $\varphi_{1} \in \Gamma$. Then, by Expression (6) of Lemma 7, we have that $\varphi_{1} \vdash \varphi_{1}$. Then, by Rule $\vee_{K}^{2}$, we have that $\varphi_{1} \vdash \varphi_{1} \vee \varphi_{2}$. Hence, by Lemma 5. we have that $\varphi_{1} \vee \varphi_{2} \in \Gamma$. Second, we prove that if $\varphi_{1} \vee \varphi_{2} \in \Gamma$ then $\varphi_{1} \in \Gamma$ or $\varphi_{2} \in \Gamma$. Assume towards a contradiction that $\varphi_{1} \notin \Gamma$ and $\varphi_{2} \notin \Gamma$. Then, because $\Gamma$ is a maximal UL-consistent set, there are $X_{1}, \ldots, X_{m} \in \Gamma$ and $Y_{1}, \ldots, Y_{n} \in \Gamma$ such that $X_{1}, \ldots, X_{m}, \varphi_{1} \vdash$ and $Y_{1}, \ldots, Y_{n}, \varphi_{2} \vdash$. Then, by rule $\mathrm{K}$ and $\mathrm{Cl}$, we have that $X_{1}, \ldots, X_{m}, Y_{1}, \ldots, Y_{n}, \varphi_{1} \vdash$ and $X_{1}, \ldots, X_{m}, Y_{1}, \ldots, Y_{n}, \varphi_{2} \vdash$. So, by the display rules, we have that $\varphi_{1} \vdash *\left(X_{1}, \ldots, X_{m}, Y_{1}, \ldots, Y_{n}\right)$ and $\varphi_{2} \vdash *\left(X_{1}, \ldots, X_{m}, Y_{1}, \ldots, Y_{n}\right)$. Then, by rule $\vee_{A}$, we have that $\varphi_{1} \vee \varphi_{2} \vdash *\left(X_{1}, \ldots, X_{m}, Y_{1}, \ldots, Y_{n}\right)$. So, by the display rules, we have that $\varphi_{1} \vee \varphi_{2}, X_{1}, \ldots, X_{m}, Y_{1}, \ldots, Y_{n} \vdash$. However, $X_{1}, \ldots, X_{m}, Y_{1}, \ldots, Y_{n}$ and $\varphi_{1} \vee \varphi_{2}$ belong to $\Gamma$. This entails that $\Gamma$ is not UL-consistent, which is impossible. Thus, either $\varphi_{1} \in \Gamma$ or $\varphi_{2} \in \Gamma$.

- $\square \varphi$ :

Assume that $\square \varphi \in \Gamma$. Then, for all $\Gamma^{\prime}$ such that $\Gamma^{\prime} \in R(\Gamma)$, we have that $\varphi \in \Gamma^{\prime}$. Therefore, $\mathcal{M}^{c}, \Gamma^{\prime} \models \varphi$ by Induction Hypothesis. So, $\mathcal{M}^{c}, \Gamma \models \square \varphi$ by definition.

Assume that $\mathcal{M}^{c}, \Gamma \models \square \varphi$. We are going to show that $\square \varphi \in \Gamma$. Let $S:=\{* \varphi\} \cup\{\varphi \mid \square \varphi \in \Gamma\}$ and assume that $S$ is UL-consistent. Then, $S$ can be extended to a maximal UL-consistent set $S^{+}$by Lemma 6 . Now, $* \varphi \in S^{+}$because $* \varphi \in S$. Now, $\varphi \notin S^{+}$, because otherwise we would have that $\varphi, * \varphi \vdash$ and $\Gamma$ would then not be UL-consistent. So, it is not the case that $\mathcal{M}^{c}, S^{+} \Vdash \varphi$ by Induction Hypothesis. Moreover, $S^{+} \in R^{c}(\Gamma)$ by definition of $R^{c}$. Hence, it is not the case that $\mathcal{M}^{c}, \Gamma \models \square \varphi$. This is impossible by assumption. So, $S$ is not UL-consistent. So, there are $\varphi_{1}, \ldots, \varphi_{n} \in S$ such that $\varphi_{1}, \ldots, \varphi_{n}, * \varphi \vdash$. Hence, $\varphi_{1}, \ldots, \varphi_{n} \vdash \varphi$ by the display rules. So, by the rules for $\square$, we have that $\square \varphi_{1}, \ldots, \square \varphi_{n} \vdash \square \varphi$. So, because $\Gamma$ is a maximal UL-consistent set of $\mathcal{L}$, it holds that $\square \varphi \in \Gamma$ by Lemma 5 .

$\bullet \diamond^{-} \varphi:$

Assume that $\mathcal{M}^{c}, \Gamma \Vdash \diamond^{-} \varphi$. We are going to show that $\diamond^{-} \varphi \in \Gamma$. By definition of $\|$, we have that there is $\Gamma^{\prime} \in \mathcal{M}^{c}$ such that $\Gamma^{\prime} \mathcal{R}^{c} \Gamma$ and $\mathcal{M}^{c}, \Gamma^{\prime} \| \varphi$. Then, by Induction Hypothesis, we have that $\varphi \in \Gamma^{\prime}$. Now, by definition of $\mathcal{R}^{c}:=\left(\mathcal{R}_{\diamond}^{c}\right)^{-}$, we have that $\diamond^{-} \varphi \in \Gamma$.

Reciprocally, assume that $\diamond^{-} \varphi \in \Gamma$ and let $S:=\{\varphi\} \cup\left\{* \psi \mid * \diamond^{-} \psi \in \Gamma\right\}$. Assume towards a contradiction that $S$ is not UL-consistent. Then, there are $* \psi_{1}, \ldots, * \psi_{n} \in S$ such that $\varphi, * \psi_{1}, \ldots, * \psi_{n} \vdash$. Then, by iterated application of the display rules and rule $\neg_{K}$, 
we have that $\varphi \vdash \psi_{1}, \ldots, \psi_{n}$. Then, $\diamond^{-} \varphi \vdash \diamond^{-} \psi_{1}, \ldots, \diamond^{-} \psi_{n}$ by the rules for $\diamond^{-}$. So, $\diamond^{-} \varphi, * \diamond^{-} \psi_{1}, \ldots, * \diamond^{-} \psi_{n} \vdash$. Because $\diamond^{-} \varphi, * \diamond^{-} \psi_{1}, \ldots, * \diamond^{-} \psi_{n} \in \Gamma$, this entails that $\Gamma$ is not UL-consistent, which contradicts our assumption. Therefore, $S$ is UL-consistent, and by Lemma 6, it can be extended into a maximal UL-consistent set $\Gamma^{\prime}$. Then, $\Gamma^{\prime}$ is such that for all $* \diamond^{-} \psi \in \Gamma$, we have that $* \psi \in \Gamma^{\prime}(*)$. Now, let $\psi \in \Gamma^{\prime}$, then we have that $* \psi \notin \Gamma^{\prime}$, because otherwise we would have $\psi, * \psi \vdash$ and $\Gamma$ would not be UL-consistent. So, by contraposition of $(*)$, we also have that $* \diamond^{-} \psi \notin \Gamma$. Because $\Gamma$ is a maximal UL-consistent set, this entails by definition that there are $X_{1}, \ldots X_{n} \in \Gamma$ such that $X_{1}, \ldots, X_{n}, * \diamond^{-} \psi \vdash$. Then, by the display rules, this entails that $X_{1}, \ldots, X_{n} \vdash \diamond^{-} \psi$. Hence, by Lemma 5 , we have that $\diamond^{-} \psi \in \Gamma$. Thus we must have that $\diamond^{-} \psi \in \Gamma$ for all $\psi \in \Gamma$. So, $\Gamma^{\prime} \mathcal{R}^{c} \Gamma$ by definition of $\mathcal{R}^{c}$. Finally, $\varphi \in \Gamma^{\prime}$, therefore we have that $\mathcal{M}^{c}, \Gamma^{\prime} \Vdash \varphi$ by Induction Hypothesis. This entails that $\mathcal{M}^{c}, \Gamma \Vdash \diamond^{-} \varphi$.

- $\varphi \otimes_{3} \chi:$

Assume that $\varphi \otimes_{3} \chi \in \Gamma^{\prime \prime}$. We must show that $\mathcal{M}^{c}, \Gamma^{\prime \prime} \| \varphi \otimes_{3} \chi$, that is, there are $\Gamma, \Gamma^{\prime} \in \mathcal{M}^{c}$ such that $\left(\Gamma, \Gamma^{\prime}, \Gamma^{\prime \prime}\right) \in \mathcal{R}^{c}, \mathcal{M}^{c}, \Gamma \Vdash \varphi$ and $\mathcal{M}^{c}, \Gamma^{\prime} \Vdash \chi$, i.e., there are $\Gamma, \Gamma^{\prime} \in \mathcal{M}^{c}$ such that $\left(\Gamma, \Gamma^{\prime}, \Gamma^{\prime \prime}\right) \in \mathcal{R}^{c}, \varphi \in \Gamma$ and $\chi \in \Gamma^{\prime}$. We construct the maximal consistent sets $\Gamma$ and $\Gamma^{\prime}$ following the steps described in the 'pseudo'-Algorithm 1 (we call it 'pseudo'-Algorithm because it is not terminating, we only introduce it in order to better explain the way we construct $\Gamma$ and $\left.\Gamma^{\prime}\right)$.

We prove that the 'pseudo'-Algorithm 1 is well-defined. To do so, we prove that for all $n \in \mathbb{N}$,

$$
\begin{aligned}
& \left(\bigwedge \Gamma_{n} \wedge \varphi_{n}\right) \quad \otimes_{3} \quad\left(\bigwedge \Gamma_{n}^{\prime} \wedge \chi_{n}\right) \in \Gamma^{\prime \prime} \quad \text { or } \\
& \left(\bigwedge \Gamma_{n} \wedge \neg \varphi_{n}\right) \quad \otimes_{3} \quad\left(\bigwedge \Gamma_{n}^{\prime} \wedge \chi_{n}\right) \in \Gamma^{\prime \prime} \quad \text { or } \\
& \left(\bigwedge \Gamma_{n} \wedge \neg \varphi_{n}\right) \quad \otimes_{3} \quad\left(\bigwedge \Gamma_{n}^{\prime} \wedge \neg \chi_{n}\right) \in \Gamma^{\prime \prime} \text { or } \\
& \left(\bigwedge \Gamma_{n} \wedge \varphi_{n}\right) \quad \otimes_{3} \quad\left(\bigwedge \Gamma_{n}^{\prime} \wedge \neg \chi_{n}\right) \in \Gamma^{\prime \prime}
\end{aligned}
$$

(The "or" is inclusive.) Expression (13) is due to the fact that for all $\varphi, \psi, \chi, \rho \in$ $\mathcal{S}\left(\mathbb{P}, \operatorname{Con}_{\varphi}\right)$, we can prove the following:

$$
\begin{aligned}
& \psi \otimes_{3} \rho \vdash(\psi \wedge \varphi) \otimes_{3}(\rho \wedge \chi) \vee(\psi \wedge \neg \varphi) \otimes_{3}(\rho \wedge \chi) \vee \\
&(\psi \wedge \neg \varphi) \otimes_{3}(\rho \wedge \neg \chi) \vee(\psi \wedge \varphi) \otimes_{3}(\rho \wedge \neg \chi)
\end{aligned}
$$

To prove Expression (14), we use Expression (10) of Lemma 7 and the fact that $\psi \otimes_{3}$ $\rho \vdash((\psi \wedge \varphi) \vee(\psi \wedge \neg \varphi)) \otimes_{3}((\rho \wedge \chi) \vee(\rho \wedge \neg \chi))$, which is itself proved by application of rules $\otimes_{K}^{3}$ and then $\otimes_{A}^{3}$ to $\psi \vdash(\psi \wedge \varphi) \vee(\psi \wedge \neg \varphi)$ and $\rho \vdash(\rho \wedge \chi) \vee(\rho \wedge \neg \chi)$. Replacing in Expression $14 \psi$ with $\bigwedge \Gamma_{n}, \rho$ with $\bigwedge \Gamma_{n}^{\prime}, \varphi$ with $\varphi_{n}$ and $\chi$ with $\chi_{n}$, and using Lemma 5 together with the fact that $\bigwedge \Gamma_{n} \otimes_{3} \bigwedge \Gamma_{n}^{\prime} \in \Gamma^{\prime \prime}$, we obtain the result of Expression $\sqrt{13}$, because $\Gamma^{\prime \prime}$ is a maximal consistent set. So, the 'pseudo'-algorithm is well-defined.

Now, we prove that $\Gamma$ and $\Gamma^{\prime}$ are maximal UL-consistent sets. By Lemma 8 , for all $n \in$ $\mathbb{N}, \Gamma_{n}$ and $\Gamma_{n}^{\prime}$ are UL-consistent, because $\bigwedge \Gamma_{n} \otimes_{3} \bigwedge \Gamma_{n}^{\prime}$ is UL-consistent since $\bigwedge \Gamma_{n} \otimes_{3}$ 


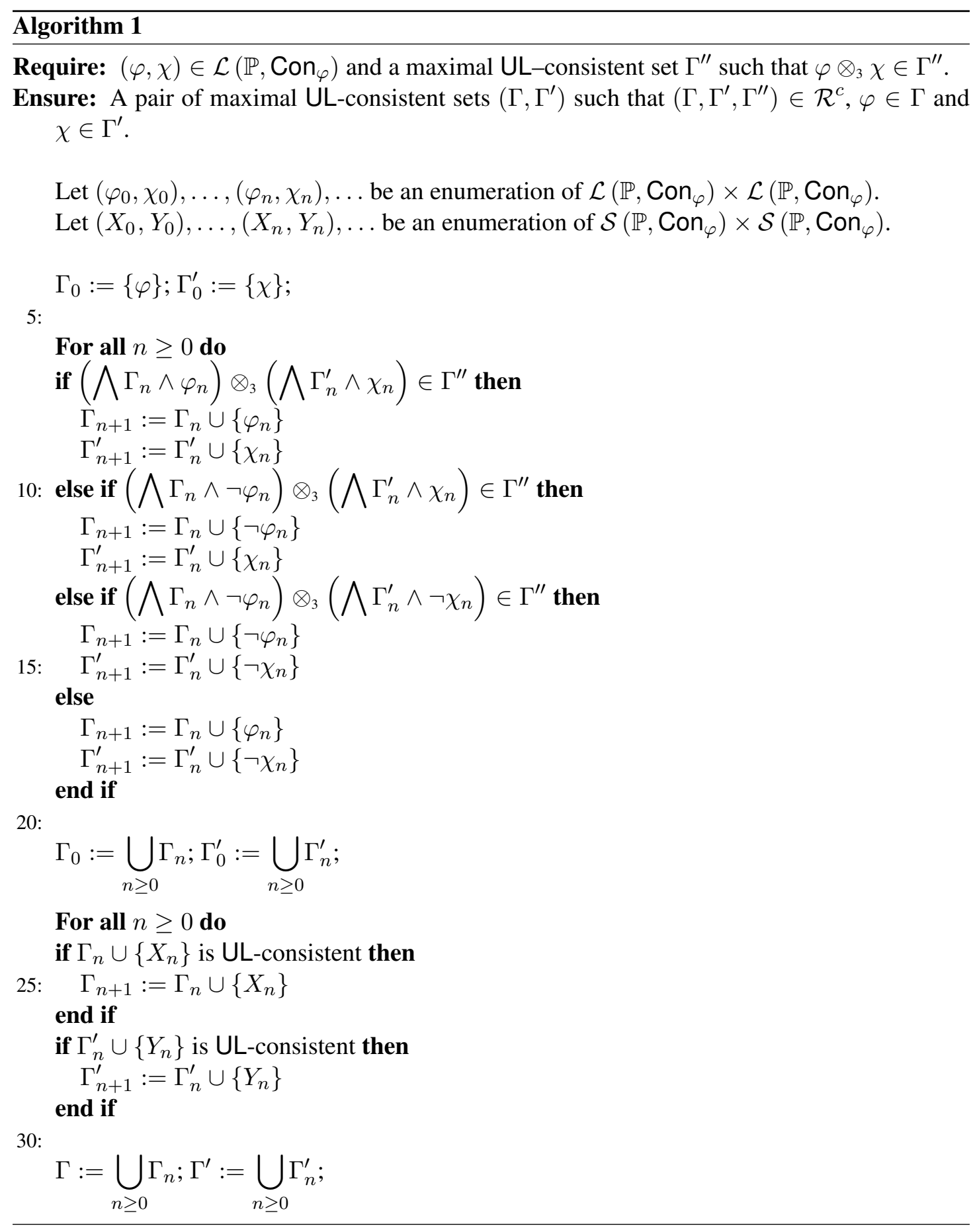


$\bigwedge \Gamma_{n}^{\prime} \in \Gamma^{\prime \prime}$ and $\Gamma^{\prime \prime}$ is UL-consistent. So, at line 21 of the 'pseudo'-algorithm, $\Gamma_{0}$ and $\Gamma_{0}^{\prime}$ are also UL-consistent, since otherwise there would be a $n$ such that $\Gamma_{n}$ and $\Gamma_{n}^{\prime}$ are not ULconsistent. Hence, $\Gamma$ and $\Gamma^{\prime}$ are also UL-consistent at the end of the 'pseudo'-algorithm by definition of the rest of the 'pseudo'-algorithm. Moreover, by construction of $\Gamma$ and $\Gamma^{\prime}$, because all pairs of $\mathcal{S}\left(\mathbb{P}, \operatorname{Con}_{\varphi}\right) \times \mathcal{S}\left(\mathbb{P}, \operatorname{Con}_{\varphi}\right)$ are enumerated, $\Gamma$ and $\Gamma^{\prime}$ are maximal consistent sets of $\mathcal{S}\left(\mathbb{P}, \operatorname{Con}_{\varphi}, \operatorname{Con}_{X}\right)$.

Finally, we need to prove that $\left(\Gamma, \Gamma^{\prime}, \Gamma^{\prime \prime}\right) \in \mathcal{R}^{c}$. To do so, it suffices to prove that for all $\varphi \in \Gamma$, all $\chi \in \Gamma^{\prime}, \varphi \otimes_{3} \chi \in \Gamma^{\prime \prime}$ by Definition 17 of the canonical model. Let $\varphi \in \Gamma$ and let $\chi \in \Gamma^{\prime}$. Then, there is $n \in \mathbb{N}$ such that $(\varphi, \chi)=\left(\varphi_{n}, \chi_{n}\right)$. Then, by definition of the algorithm, we must have that $\left(\Gamma_{n} \wedge \varphi\right) \otimes_{3}\left(\Gamma_{n}^{\prime} \wedge \chi\right) \in \Gamma^{\prime \prime}$. Therefore, because $\Gamma_{n} \wedge \varphi \vdash \varphi$ and $\Gamma_{n}^{\prime} \wedge \chi \vdash \chi$, we have that $\left(\Gamma_{n} \wedge \varphi\right) \otimes_{3}\left(\Gamma_{n}^{\prime} \wedge \chi\right) \vdash \varphi \otimes_{3} \chi$ by application of rules $\otimes_{K}^{3}$ and then $\otimes_{A}^{3}$. Thus, $\varphi \otimes_{3} \chi \in \Gamma^{\prime \prime}$ by Lemma 5 , and therefore $\left(\Gamma, \Gamma^{\prime}, \Gamma^{\prime \prime}\right) \in \mathcal{R}^{c}$.

Now, we prove the converse, that is, if $\mathcal{M}^{c}, \Gamma^{\prime \prime} \Vdash \varphi \otimes_{3} \chi$, then $\varphi \otimes_{3} \chi \in \Gamma^{\prime \prime}$. By definition of $\otimes_{3}$, we have that there are $\Gamma$ and $\Gamma^{\prime}$ such that $\left(\Gamma, \Gamma^{\prime}, \Gamma^{\prime \prime}\right) \in \mathcal{R}^{c}, \mathcal{M}^{c}, \Gamma \Vdash \varphi$ and $\mathcal{M}^{c}, \Gamma^{\prime} \Vdash \chi$. So, by Induction Hypothesis, we have that $\varphi \in \Gamma$ and $\chi \in \Gamma^{\prime}$. So, by definition of $\mathcal{R}^{c}\left(=\mathcal{R}_{\otimes}^{c}\right)$, we must have that $\varphi \otimes_{3} \chi \in \Gamma^{\prime \prime}$, since $\left(\Gamma, \Gamma^{\prime}, \Gamma^{\prime \prime}\right) \in \mathcal{R}^{c}$. This completes the proof.

- $\chi \supset_{1} \psi$ :

Assume that $\chi \supset_{1} \psi \in \Gamma$. Then, for all $\Gamma^{\prime}, \Gamma^{\prime \prime}$ such that $\left(\Gamma, \Gamma^{\prime}, \Gamma^{\prime \prime}\right) \in \mathcal{R}^{c}$, if $\chi \in \Gamma^{\prime}$ then $\psi \in \Gamma^{\prime \prime}$. That is, for all $\Gamma^{\prime}, \Gamma^{\prime \prime}$ such that $\left(\Gamma, \Gamma^{\prime}, \Gamma^{\prime \prime}\right) \in \mathcal{R}^{c}$, if $\mathcal{M}^{c}, \Gamma^{\prime} \Vdash \chi$ then $\mathcal{M}^{c}, \Gamma^{\prime \prime} \Vdash \psi$ by Induction Hypothesis. That is, $\mathcal{M}^{c}, \Gamma \Vdash \chi \supset_{1} \psi$.

Reciprocally, assume that $\mathcal{M}^{c}, \Gamma \Vdash \chi \supset_{1} \psi$ and assume towards a contradiction that $\chi \supset_{1}$ $\psi \notin \Gamma$. We are going to show that, in that case, there are $\Gamma^{\prime}, \Gamma^{\prime \prime} \in \mathcal{M}^{c}$ such that $\left(\Gamma, \Gamma^{\prime}, \Gamma^{\prime \prime}\right) \in \mathcal{R}^{c}$ such that $\mathcal{M}^{c}, \Gamma^{\prime} \Vdash \chi$ but it is not the case that $\mathcal{M}^{c}, \Gamma^{\prime \prime} \Vdash \psi$, contradicting the assumption.

1. Assume towards a contradiction that $S:=\left\{\varphi \mid \chi \supset_{1} \varphi \in \Gamma\right\} \cup\{* \psi\}$ is not $\mathrm{UL}^{*}$ consistent. Then, there are $\varphi_{1}, \ldots, \varphi_{n} \in S$ such that $* \psi, \varphi_{1}, \ldots, \varphi_{n} \vdash$. Hence, $\varphi_{1}, \ldots, \varphi_{n} \vdash \psi$ by the display rules. So, $\chi \supset_{1} \varphi_{1}, \ldots, \chi \supset_{1} \varphi_{n} \vdash \chi \supset_{1} \psi$ by application of $\supset_{L}$ and then $\supset_{R}$ to $\varphi_{1}, \ldots, \varphi_{n} \vdash \psi$ and $\chi \vdash \chi$. Then, by application of Lemma 5 , we have that $\chi \supset_{1} \psi \in \Gamma$. This is impossible. Therefore, $S$ is UL ${ }^{*}$-consistent and by Lemma 6 it can be extended into a maximal consistent set called $\Gamma^{\prime \prime}$.

2. Now, let $S_{\chi}:=\{\chi\} \cup\left\{* \rho \mid\right.$ there is $\left.\psi \notin \Gamma^{\prime \prime}, \rho \supset_{1} \psi \in \Gamma\right\}$. Assume that $S_{\chi}$ is not $\mathrm{UL}^{*}$-consistent. Then, there are $* \rho_{1}, \ldots, * \rho_{n} \in S_{\chi}$ such that $\chi, * \rho_{1}, \ldots, * \rho_{n} \vdash$. So, $\chi \vdash \rho_{1}, \ldots, \rho_{n}(*)$ by the display rules. Therefore, we have that $\rho_{1} \supset_{1} \bigvee_{i} \psi_{i}, \ldots, \rho_{n} \supset_{1}$ $\bigvee_{i} \psi_{i} \vdash \chi \supset_{1} \bigvee_{i} \psi_{i}(* *)$ by iterated application of the rules $\supset_{A}^{1}$ and $\supset_{K}^{1}$ to $(*)$ and $\bigvee_{i} \psi_{i} \vdash \bigvee_{i} \psi_{i}$. Now, by application of Lemma 5 , we have that $\rho_{i} \supset_{1} \bigvee_{i} \psi_{i} \in \Gamma$, because by definition of $S_{\chi}$ we have that $\rho_{i} \supset_{1} \psi_{i} \in \Gamma$ for all $i \in\{1, \ldots, n\}$. Then, again by application of Lemma 5 to $(* *)$, 
we have that $\chi \supset_{1} \bigvee_{i} \psi_{i} \in \Gamma$. Hence, $\bigvee_{i} \psi_{i} \in S$ by definition of $S$. So, $\bigvee_{i} \psi_{i} \in \Gamma^{\prime \prime}$ by definition of $\Gamma^{\prime \prime}$. Therefore, by Lemma 12, we have that there must exist an $i$ such that $\psi_{i} \in \Gamma^{\prime \prime}$. This is impossible by definition of $S_{\chi}$. Therefore our initial assumption was wrong and $S_{\chi}$ must be $\mathrm{UL}^{*}$-consistent. So, it can be extended into a maximal $\mathrm{UL}^{*}$-consistent set called $\Gamma^{\prime}$.

Finally, we must check that $\left(\Gamma, \Gamma^{\prime}, \Gamma^{\prime \prime}\right) \in \mathcal{R}^{c}$. Let $\rho \supset_{1} \varphi \in \Gamma$ and assume that $\rho \in \Gamma^{\prime}$ but $\varphi \notin \Gamma^{\prime \prime}$. Then, by definition of $\Gamma^{\prime}, \neg \rho \in \Gamma^{\prime}$, which contradicts the fact that $\rho \in \Gamma^{\prime}$. So, $\varphi \in \Gamma^{\prime \prime}$. Hence, there are $\left(\Gamma, \Gamma^{\prime}, \Gamma^{\prime \prime}\right) \in \mathcal{R}^{c}$ such that $\chi \in \Gamma^{\prime}$ and $\neg \psi \in \Gamma^{\prime \prime}$. So, there are $\left(\Gamma, \Gamma^{\prime}, \Gamma^{\prime \prime}\right) \in \mathcal{R}^{c}$ such that $\mathcal{M}^{c}, \Gamma^{\prime} \Vdash \chi$ and $\mathcal{M}^{c}, \Gamma^{\prime \prime} \Vdash \neg \psi$ by Induction Hypothesis. Therefore, it is not the case that $\mathcal{M}^{c}, \Gamma \Vdash \chi \supset_{1} \psi$, which contradicts our assumption. We have reached a contradiction, so, finally, $\chi \supset_{1} \psi \in \Gamma$.

- $\varphi \subset_{2} \psi$ :

Assume that $\psi \subset_{2} \varphi \in \Gamma^{\prime}$. Then, for all $\Gamma, \Gamma^{\prime \prime} \in \mathcal{M}^{c}$ such that $\left(\Gamma, \Gamma^{\prime}, \Gamma^{\prime \prime}\right) \in \mathcal{R}^{c}$, if $\psi \in \Gamma$ then $\varphi \in \Gamma^{\prime \prime}$. Then, for all $\Gamma, \Gamma^{\prime \prime} \in \mathcal{M}^{c}$ such that $\left(\Gamma, \Gamma^{\prime}, \Gamma^{\prime \prime}\right) \in \mathcal{R}^{c}$, if $\mathcal{M}^{c}, \Gamma \| \psi$ then $\mathcal{M}^{c}, \Gamma^{\prime \prime} \Vdash \varphi$. That is, $\mathcal{M}^{c}, \Gamma^{\prime} \Vdash \psi \subset_{2} \varphi$.

Reciprocally, assume that $\mathcal{M}^{c}, \Gamma^{\prime} \Vdash \psi \subset_{2} \varphi$. Assume towards a contradiction that $\psi \subset_{2} \varphi \notin$ $\Gamma^{\prime}$. We are going to show that, in that case, there exist $\Gamma, \Gamma^{\prime \prime} \in \mathcal{M}^{c}$ such that $\left(\Gamma, \Gamma^{\prime}, \Gamma^{\prime \prime}\right) \in \mathcal{R}^{c}$ and $\mathcal{M}^{c}, \Gamma \Vdash \psi$, but it is not the case that $\mathcal{M}^{c}, \Gamma^{\prime \prime} \Vdash \varphi$, contradicting the assumption.

1. Let $S^{\prime \prime}:=\{* \varphi\} \cup\left\{\varphi \mid \psi \subset_{2} \varphi \in \Gamma^{\prime}\right\}$ and assume that $S^{\prime \prime}$ is not $U L^{*}$-consistent. Then, there are $\varphi_{1}, \ldots, \varphi_{n} \in S^{\prime \prime}$ such that $* \varphi, \varphi_{1}, \ldots, \varphi_{n} \vdash$. So, $\varphi_{1}, \ldots, \varphi_{n} \vdash \varphi$ by the display rules. Then, by application of $\subset_{L}$ and then $\subset_{R}$ to $\varphi_{1}, \ldots, \varphi_{n} \vdash \varphi$ and $\psi \vdash \psi$, we have that $\psi \subset_{2} \varphi_{1}, \ldots, \psi \subset_{2} \varphi_{n} \vdash \psi \subset_{2} \varphi$. But $\psi \subset_{2} \varphi_{1}, \ldots, \psi \subset_{2} \varphi_{n} \in \Gamma^{\prime}$. So $\psi \subset_{2} \varphi \in \Gamma^{\prime}$ by application of Lemma 5. This is impossible by assumption. Therefore, $S^{\prime \prime}$ is UL ${ }^{*}$-consistent. Then, $S^{\prime \prime}$ can be extended into a maximal $\mathrm{UL}^{*}$-consistent set $\Gamma^{\prime \prime} \in \mathcal{M}^{c}$ by Lemma 6 .

2. Now, let $S:=\{\psi\} \cup\left\{* \varphi \mid\right.$ there is $\chi \notin \Gamma^{\prime \prime}$ such that $\left.\chi \subset_{2} \varphi \in \Gamma^{\prime}\right\}$. Assume that $S$ is not $\mathrm{UL}^{*}$-consistent. Then, there are $* \varphi_{1}, \ldots, * \varphi_{n} \in S$ such that $\psi, * \varphi_{1}, \ldots, * \varphi_{n} \vdash$. Then, $\psi \vdash \varphi_{1}, \ldots, \varphi_{n}$ by the display rules. So, $\psi \vdash \bigvee_{i} \varphi_{i}$ by iterated application of rule $\vee_{K}$, the display rules and WI. Let $\chi_{1}, \ldots, \chi_{n}$ be the formulas of $\Gamma^{\prime \prime}$ associated to $\varphi_{1}, \ldots, \varphi_{n}$ through $S$ (for all $i \in\{1, \ldots, n\}$, we have $\chi_{i} \subset_{2} \varphi_{i} \in \Gamma^{\prime}$ ). Then, because $\bigvee_{i} \chi_{i} \vdash \bigvee_{i} \chi_{i}$, we have that $\psi,,_{3} \bigvee_{i} \chi_{i} \subset_{2} \bigvee_{i} \varphi_{i} \vdash \bigvee_{i} \chi_{i}$ by rule $\subset_{A}^{2}$. Therefore, by rule $\subset_{K}^{2}$,

$$
\bigvee_{i} \chi_{i} \subset_{2} \bigvee_{i} \varphi_{i} \vdash \psi \subset_{2} \bigvee_{i} \chi_{i}
$$

Moreover, by Expression [11, it holds that $\bigvee_{i} \chi_{i} \subset_{2} \varphi_{1}, \ldots, \bigvee_{i} \chi_{i} \subset_{2} \varphi_{n} \vdash \bigvee_{i} \chi_{i} \subset_{2} \bigvee_{i} \varphi_{i}$ $(* *)$. Now, for all $i$, one can easily prove that $\chi_{i} \subset_{2} \varphi_{i} \vdash \bigvee_{i} \chi_{i} \subset_{2} \varphi_{i}$. So, by application of the 
Cut rule, we have

$$
\chi_{1} \subset_{2} \varphi_{1}, \ldots, \chi_{n} \subset_{2} \varphi_{n} \vdash \bigvee_{i} \chi_{i} \subset_{2} \bigvee_{i} \varphi_{i}
$$

Applying again the cut rule to Expressions (15) and (16), we obtain

$$
\chi_{1} \subset_{2} \varphi_{1}, \ldots, \chi_{n} \subset_{2} \varphi_{n} \vdash \psi \subset_{2} \bigvee_{i} \chi_{i}
$$

Now, for all $i \in\{1, \ldots, n\}$, we have $\chi_{i} \subset_{2} \varphi_{i} \in \Gamma^{\prime}$. Therefore, by application of Lemma 5 to Expression (17), we have that $\psi \subset_{2} \bigvee_{i} \chi_{i} \in \Gamma^{\prime}$. Then, by application of Lemma 5 to (15), we have that $\psi \subset_{2} \bigvee_{i} \chi_{i} \in \Gamma^{\prime}$. So, $\bigvee_{i} \chi_{i} \in \Gamma^{\prime \prime}$ by definition of $S^{\prime \prime}$. Thus, by Lemma 12 , there must be a $i \in\{1, \ldots, n\}$ such that $\chi_{i} \in \Gamma^{\prime \prime}$. However, $\chi_{i} \notin \Gamma^{\prime \prime}$ for all $i$ by definition of $S$. Hence, we reach a contradiction. Therefore, $S$ must be $U L^{*}$-consistent. So, it can be extended to a maximal $U L^{*}$-consistent set $\Gamma \in \mathcal{M}^{c}$ by Lemma 6

Now, we prove that we have $\left(\Gamma, \Gamma^{\prime}, \Gamma^{\prime \prime}\right) \in \mathcal{R}^{c}$. Let $\psi \subset_{2} \varphi \in \Gamma^{\prime}$ and assume towards a contradiction that $\varphi \in \Gamma$ but $\psi \notin \Gamma^{\prime \prime}$. Then, $* \varphi \in S$ by definition of $S$, so $* \varphi \in \Gamma$. This is impossible because $\varphi \in \Gamma$ and $\Gamma$ is a maximal $\mathrm{UL}^{*}$-consistent set. So, if $\varphi \in \Gamma$ then $\psi \in \Gamma^{\prime \prime}$. Therefore, for all $\psi \subset_{2} \varphi \in \Gamma^{\prime}$, if $\varphi \in \Gamma$ then $\psi \in \Gamma^{\prime \prime}$. Hence, $\left(\Gamma, \Gamma^{\prime}, \Gamma^{\prime \prime}\right) \in \mathcal{R}^{c}$.

Moreover, $\varphi \in \Gamma$ and $\psi \notin \Gamma^{\prime \prime}$, so by Induction Hypothesis, $\mathcal{M}^{c}, \Gamma \Vdash \psi$ and not $\mathcal{M}^{c}, \Gamma^{\prime \prime} \Vdash \varphi$. Therefore, we do not have that $\mathcal{M}^{c}, \Gamma^{\prime} \Vdash \psi \subset_{2} \varphi$, which is impossible by assumption. So, finally, $\psi \subset_{2} \varphi \in \Gamma^{\prime}$.

The proofs for the cases $\psi \otimes_{\mathrm{i}} \chi, \chi \supset_{\mathrm{j}}, \psi \subset_{\mathrm{k}} \chi$, where $(i, j, k) \in\{(1,2,3),(2,3,1)\}$, are dealt with similarly and the proofs can be easily adapted. So, we do not repeat the proofs for these cases.

Finally, we prove Theorem 2 .

Proof of Theorem 2 The proof of soundness is without difficulty, so we only prove strong completeness, i.e., we prove that for all sets $\Gamma \subseteq \mathcal{C}\left(\operatorname{Con}_{\varphi}\right)$ and all $S:=X \vdash Y \in \mathcal{C}\left(\operatorname{Con}_{\varphi}\right)$, if $\Gamma \Vdash S$ holds then $\Gamma \vdash S$ is provable in UL. We reason by contraposition. Assume towards a contradiction that it is not the case that $\Gamma \vdash S$. Then, it is not the case that there is a proof of $S$ in UL from $\Gamma$. Thus, it is not the case that $X, * Y \vdash$ can be proved in $\mathrm{UL}+\Gamma$. So, it is not the case that $\tau_{1}(X, * Y) \vdash$ can be proved in $\mathrm{UL}+\Gamma$, by Expression (9). That is, $E:=\left\{\tau_{1}(X, * Y)\right\}$ is $\mathrm{UL}+\Gamma$-consistent. So, by Lemma 6 (where UL-consistency is replaced by $\mathrm{UL}+\Gamma$-consistency), it can be extended into a maximal UL $+\Gamma$-consistent set $\Gamma^{\prime}$. Now, $\Gamma^{\prime}$ is also UL-consistent, so it is a possible world of $\mathcal{M}^{c}$ such that $E \cup \Gamma \subseteq \Gamma^{\prime}$. So, by Lemma 10, for all $Z \in \Gamma^{\prime}$, we have that $\mathcal{M}^{c}, \Gamma^{\prime} \|^{A} Z$. Then, there is a pointed substructural model $\left(\mathcal{M}^{c}, \Gamma^{\prime}\right)$ such that $\mathcal{M}^{c}, \Gamma^{\prime} \|^{A} \Gamma$ and $\mathcal{M}^{c}, \Gamma^{\prime} \|^{A} \tau_{1}(X, * Y)$, i.e., such that $\mathcal{M}^{c}, \Gamma^{\prime} \|^{A} \Gamma$ and $\mathcal{M}^{c}, \Gamma^{\prime} \|^{A} X, * Y$ by Expression (3) in the proof of Proposition 1 . That is, it is not the case that $\Gamma \Vdash S$, which contradicts our assumption. Thus, $\Gamma \vdash S$. 


\section{From Update Logic to Substructural Logics}

So far we have not imposed any constraint on the ternary or the binary relation of the substructural frames we have considered. We are now going to show that our soundness and (strong) completeness results can be extended to many other logics, whose accessibility relations satisfy specific conditions, by adding some appropriate corresponding structural rules to our basic display calculus UL. We will thereby define novel display calculi for these extended logics, some of them being well-known substructural logics.

In this section we consider logical languages without Boolean negation.

Notation 3. In the rest of this article, $A$ is any set of structural rules from Figure 8 (it could in fact be any rule of [61] Table 11.1] mentioning the connectives of Con $\left.{ }_{X}\right)$. Moreover, in the rest of this article, $\mathcal{E}_{A}$ (respectively $\mathcal{F}_{A}$ ) is the class of pointed substructural models (resp. frames) satisfying the corresponding conditions on frames (also given in Figure 8) and whose accessibility relations are all plump.

\subsection{Admissibility of the Cut Rule}

Theorem 11 (Strong cut elimination). The display calculus $U L+A$ is a proper display calculus. Hence, $U L+A$ enjoys strong cut-elimination and therefore the cut rule is an admissible rule of $U L+A$.

Proof. The logical and structural rules of UL were already considered in the proof of Theorem 4. So, we only need to check the structural rules of Figure 8 . A rapid inspection shows that they indeed respect the eight conditions $(C 1)-(C 8)$ of Definition 15 . Therefore, $\mathrm{UL}+A$ is a proper display calculus, for any set $A$ of structural rules from Figure 8 . It enjoys strong cut-elimination because of [78, Theorem 23].

\subsection{More Completeness Results}

We are going to extend the completeness result of Corollary 1 to richer proof systems including various sorts of structural inference rules. For that, we need to prove a series of lemmata.

Lemma 12. Let $C_{\varphi} \subseteq \operatorname{Con}_{\varphi}-\{\neg\}$. Let $\Gamma$ be a maximal UL-consistent set. Then, $\Gamma$ satisfies the conditions of a non-trivial prime theory of $U L\left(C_{\varphi}\right)$ (as defined in [61] Def. 5.6]). That is, for all $\varphi, \psi \in \mathcal{L}\left(\mathbb{P}, C_{\varphi}\right)$, the following hold:

1. $\perp \notin \Gamma$ and $\top \in \Gamma$;

2. If $\varphi \vdash \psi$ and $\varphi \in \Gamma$, then $\psi \in \Gamma$;

3. If $\varphi \in \Gamma$ and $\psi \in \Gamma$ then $\varphi \wedge \psi \in \Gamma$;

4. If $\varphi \vee \psi \in \Gamma$ then either $\varphi \in \Gamma$ or $\psi \in \Gamma$.

Reciprocally, every non-trivial prime theory of UL can be extended into a maximal ULconsistent set. 


$$
\begin{aligned}
& \frac{X,_{{ }_{\mathrm{i}}}\left(Y{ }_{\mathrm{i}_{\mathrm{i}}} Z\right) \vdash U}{\left(X,_{{ }_{\mathrm{i}}} Y\right){ }_{, \mathrm{i}} Z \vdash U} \\
& \mathcal{R}_{i}(x y) x w \rightarrow \mathcal{R}_{i} x(y z) w \\
& \frac{\left(X,{ }_{{ }_{\mathrm{i}}} Y\right)_{{ }_{\mathrm{i}}} Z \vdash U}{X{ }_{{ }_{\mathrm{i}}}\left(Y{ }_{{ }_{\mathrm{i}}} Z\right)-U} \\
& \frac{\left(X,{ }_{\mathrm{i}} Z\right){ }_{,_{\mathrm{i}}} Y \vdash U}{\left(X,{ }_{\mathrm{i}} Y\right){ }_{\mathrm{i}} Z \vdash U} \\
& \frac{Y{ }_{,} X \vdash U}{X{ }_{, i} Y \vdash U} \\
& \frac{\left(X,_{\mathrm{i}} Y\right)_{,_{\mathrm{i}}} Y \vdash U}{X{ }_{\mathrm{r}_{\mathrm{i}}} Y \vdash U} \\
& \frac{X{ }_{,} X \vdash U}{X \vdash U} \\
& \frac{X \vdash U}{X{ }_{, i} X \vdash U} \\
& \frac{X \vdash U}{X{ }_{, \mathrm{i}} Y \vdash U} \\
& \frac{X \vdash U}{Y{ }_{,} X \vdash U} \\
& \frac{\bullet X \vdash U}{X \vdash U} \\
& \frac{\bullet X \vdash U}{\bullet \cdot X \vdash U} \\
& \mathcal{R}_{i} x(y z) w \rightarrow \mathcal{R}_{i}(x y) z w \\
& \mathcal{R}_{i}(x z) y w \rightarrow \mathcal{R}_{i}(x y) z w \\
& \mathcal{R}_{i} x y z \rightarrow \mathcal{R}_{i} y x z \\
& \mathcal{R}_{i} x y z \rightarrow \mathcal{R}_{i}(x y) y z \\
& \mathcal{R}_{i} x x x \\
& \mathcal{R}_{i} x x y \rightarrow x \sqsubseteq y \\
& \mathcal{R}_{i} x y z \rightarrow x \sqsubseteq z \\
& \mathcal{R}_{i} y x z \rightarrow x \sqsubseteq z \\
& x R x \\
& x R y \wedge y R z \rightarrow x R z
\end{aligned}
$$

where $x, y, z, w$ range over points of a substructural frame $\mathcal{F}=(\mathcal{P}, R, \mathcal{R})$, and we use the following notations:

$$
\begin{array}{rll}
\mathcal{R}_{i} x y z & \text { iff } & \sigma_{i}(x, y, z) \in \mathcal{R} \\
\mathcal{R}_{i}(x y) z w & :=\quad \exists u\left(\mathcal{R}_{i} x y u \wedge \mathcal{R}_{i} u z w\right) \\
\mathcal{R}_{i} x(y z) w & :=\quad \exists u\left(\mathcal{R}_{i} y z u \wedge \mathcal{R}_{i} x u w\right)
\end{array}
$$

Figure 8: Conditions for Structural Rules, given Plump Relations 
Proof. We prove each item:

1. It follows from the definition of UL-consistency (for $\perp$ ) and from Lemma 5 (for $\top$ );

2. It is a direct consequence of Lemma 5 ;

3. By Expression (6), we have that $\varphi_{1} \vdash \varphi_{1}$ and $\varphi_{2} \vdash \varphi_{2}$. So, by the rule $\wedge_{K}$, we have that $\varphi_{1}, \varphi_{2} \vdash \varphi_{1} \wedge \varphi_{2}$. But $\varphi_{1}, \varphi_{2} \in \Gamma$. Therefore, by Lemma 5 , we have that $\varphi_{1} \wedge \varphi_{2} \in \Gamma$.

4. Assume towards a contradiction that $\varphi \notin \Gamma$ and $\psi \notin \Gamma$. Then, because $\Gamma$ is a maximal UL-consistent set, there are $X_{1}, \ldots, X_{m} \in \Gamma$ and $Y_{1}, \ldots, Y_{n} \in \Gamma$ such that $X_{1}, \ldots, X_{m}, \varphi \vdash$ and $Y_{1}, \ldots, Y_{n}, \psi \vdash$. Then, by rule $\mathrm{K}$ and $\mathrm{Cl}$, we have that $X_{1}, \ldots, X_{m}, Y_{1}, \ldots, Y_{n}, \varphi \vdash$ and $X_{1}, \ldots, X_{m}, Y_{1}, \ldots, Y_{n}, \psi \vdash$. So, by the display rules, we have that $\varphi \vdash *\left(X_{1}, \ldots, X_{m}, Y_{1}, \ldots, Y_{n}\right)$ and $\psi \vdash *\left(X_{1}, \ldots, X_{m}, Y_{1}, \ldots, Y_{n}\right)$. Then, by rule $\vee_{A}$, we have that $\varphi \vee \psi \vdash *$ $\left(X_{1}, \ldots, X_{m}, Y_{1}, \ldots, Y_{n}\right)$. So, again by the display rules, we have that $\varphi \vee$ $\psi, X_{1}, \ldots, X_{m}, Y_{1}, \ldots, Y_{n} \vdash$. However, $X_{1}, \ldots, X_{m}, Y_{1}, \ldots, Y_{n}$ and $\varphi \vee \psi$ belong to $\Gamma$. This entails that $\Gamma$ is not UL-consistent, which is impossible. Thus, either $\varphi \in \Gamma$ or $\psi \in \Gamma$.

Reciprocally, let $\Gamma$ be a non-trivial prime theory of UL. Then, by [61, Definition. 5.6], $\Gamma$ is a subset of $\mathcal{L}=\mathcal{L}\left(\mathrm{C}_{\varphi}\right)$. We are going to prove that $\Gamma$ is a maximal UL-consistent subset of $\mathcal{L}$. First, assume towards a contradiction that $\Gamma$ is not $U L-$ consistent. Then, by definition, there are $\varphi_{1}, \ldots, \varphi_{n} \in \mathcal{L}$ such that $\varphi_{1}, \ldots, \varphi_{n} \vdash$. Then, by the structural rules and the rules $\wedge_{A}^{1}, \wedge_{A}^{2}$, we have that $\varphi_{1} \wedge \ldots \wedge \varphi_{n} \vdash \perp$. Now, by condition 3 , we have that $\varphi_{1} \wedge \ldots \wedge \varphi_{n} \in \Gamma$, and, by condition 2 , we then have that $\perp \in \Gamma$, which is impossible by assumption. So, $\Gamma$ is UL-consistent.

Definition 19 (Filtrated canonical substructural frame). Let $\mathrm{C}_{\varphi} \subseteq \operatorname{Con}_{\varphi}-\{\neg\}$. The filtrated canonical substructural frame (associated to $\left.\mathrm{C}_{\varphi}\right)$, denoted $\overline{\mathcal{F}^{c}}:=\left(\left(\overline{P^{c}}, \sqsubseteq^{c}\right), \overline{R^{c}}, \overline{\mathcal{R}^{c}}\right)$ is the frame of the substructural model $\overline{\mathcal{M}^{c}}$ obtained by the largest filtration of the canonical substructural model $\mathcal{M}^{c}=\left(P^{c}, R^{c}, \mathcal{R}^{c}, \mathcal{I}^{c}\right)$ associated to $\mathrm{UL}+A$ by the set of formulas $\mathcal{L}\left(\mathbb{P}, \mathrm{C}_{\varphi}\right)$, and such that

$$
\bar{x} \sqsubseteq^{c} \bar{y} \text { iff } \quad x \cap \mathcal{L}\left(\mathbb{P}, \mathrm{C}_{\varphi}\right) \subseteq y \cap \mathcal{L}\left(\mathbb{P}, \mathrm{C}_{\varphi}\right) .
$$

We recall that the largest filtration is defined in [61, Definition 14.8] and [61, Example 14.10]. Moreover, by the filtration property [61, Lemma 14.9], we have that for all $\varphi \in \mathcal{L}\left(\mathbb{P}, \mathrm{C}_{\varphi}\right)$

$$
\mathcal{M}^{c}, x \Vdash \varphi \text { iff } \overline{\mathcal{M}^{c}}, \bar{x} \Vdash \varphi .
$$

Then, we have the following:

Lemma 13. Let $C_{\varphi} \subseteq \operatorname{Con}_{\varphi}-\{\neg\}$. The filtrated canonical substructural frame is isomorphic to the canonical frame $\mathcal{F}:=((P, \sqsubseteq), R, \mathcal{R})$ associated to the proof system $U L\left(C_{\varphi}\right)+A$ (as defined in [61] Definition 11.26]). 
Proof. We define two mappings $\bar{f}: P \rightarrow \overline{P^{c}}$ and $f: \overline{P^{c}} \rightarrow P$ as follows: $\bar{f}(x):=\bar{y}$, where $y$ is a maximal UL-consistent subset of $\mathcal{L}\left(\operatorname{Con}_{\varphi}, \operatorname{Con}_{X}\right)$ extending $x$, and $f(\bar{x}):=x \cap \mathcal{L}\left(\mathbb{P}, \mathrm{C}_{\varphi}\right)$.

The mappings $f$ and $\bar{f}$ are well-defined. Indeed, for all $y, z \in \bar{x}$, we have that $y \cap$ $\mathcal{L}\left(\mathbb{P}, \mathrm{C}_{\varphi}\right)=z \cap \mathcal{L}\left(\mathbb{P}, \mathrm{C}_{\varphi}\right)$ by definition of a filtration. Moreover, if $x \in P^{c}$, then $x \cap \mathcal{L}\left(\mathbb{P}, \mathrm{C}_{\varphi}\right)$ is a non-trivial prime theory by Lemma 12 , so $\bar{f}(\bar{x}) \in P$. Besides, for every non-trivial prime theory $x \in P, x$ can be extended into a maximal UL-consistent set of $\mathcal{L}\left(\operatorname{Con}_{\varphi}, \operatorname{Con}_{X}\right)$ by Lemma 12. Finally, we can easily check that $f \circ \bar{f}=\bar{f} \circ f=I d$. So, $f$ and $\bar{f}$ are bijections.

Then, by definition of the largest filtration and by definition of $\mathcal{R}^{c}$ and $R^{c}$, we have that for all $x, y, z \in P, \overline{\mathcal{R}^{c} f}(x) \bar{f}(y) \bar{f}(z)$ iff $\mathcal{R} x y z$, and $\overline{R^{c}} f(x) \bar{f}(y)$ iff $R x y$. Hence, $\bar{f}$ is an isomorphism between $\mathcal{F}$ and $\overline{\mathcal{F}}^{c}$. Finally, again because of Expression (18), we have that the image of $\complement^{c}$ by $f$ is $\sqsubseteq$, because both $\sqsubseteq$ and $\bigsqcup^{c}$ correspond to the inclusion between points.

Lemma 14. Let $\{\vee, \wedge\} \subseteq C_{\varphi} \subseteq \operatorname{Con}_{\varphi}-\{\neg\}$. Then, $\operatorname{UL}\left(C_{\varphi}\right)+A$ is sound and strongly complete for the logic $\left(\mathcal{L}\left(\mathbb{P}, C_{\varphi}\right), \mathcal{E}_{A}, \Vdash\right)$.

Proof. The proof of soundness is already given in [61, Theorem 11.20] since substructural models are specific kinds of models (as defined in [61]). So, we only prove the completeness part of the theorem, which is essentially based on the results of [61]. We must prove that for all sets $\Gamma \subseteq \mathcal{L}\left(\mathbb{P}, \mathrm{C}_{\varphi}\right)$ and all $\varphi \in \mathcal{L}\left(\mathbb{P}, \mathrm{C}_{\varphi}\right)$, if $\Gamma \Vdash \varphi$ holds then $\Gamma \vdash \varphi$ is provable in $\mathrm{UL}\left(\mathrm{C}_{\varphi}\right)+A$. Like for the proof of Theorem 2, this boils down to prove that for every $\mathrm{UL}\left(\mathrm{C}_{\varphi}\right)+A$-consistent set $S$ of $\mathcal{L}\left(\mathbb{P}, \mathrm{C}_{\varphi}\right)$-formulas, we can find a pointed substructural model $(\mathcal{M}, x) \in \mathcal{E}_{A}$ such that $\mathcal{M}, x \Vdash S$. Let $\left(\overline{\mathcal{M}^{c}}, \bar{x}\right)$ be the largest filtration of the canonical substructural model $\left(\mathcal{M}^{c}, x\right)$ associated to $\mathrm{UL}+A$ by the formulas of $\mathcal{L}\left(\mathbb{P}, \mathrm{C}_{\varphi}\right)$, where $x$ is a maximal $\mathrm{UL}+A$-consistent set extending $S$. We are going to show that $\left(\overline{\mathcal{M}^{c}}, \bar{x}\right)$ actually satisfies this condition. First, we need to prove that the underlying frame $\overline{\mathcal{F}^{c}}$ of $\overline{\mathcal{M}^{c}}$, that is the filtrated canonical substructural frame, satisfies the conditions of $A$. By [61, Corollary 11.36], the conditions corresponding to the structural rules of $A$ hold in the canonical frame $\mathcal{F}$. Using this very result and Lemma 13 , we finally have that the frame $\overline{\mathcal{F}^{c}}$ satisfies the conditions of $A$. Moreover, by [61, Lemma 11.28], the accessibility relations of $\mathcal{F}$ are plump. Thus, the accessibility relations of $\overline{\mathcal{F}^{c}}$ are also plump (because $\mathcal{F}$ and $\overline{\mathcal{F}^{c}}$ are isomorphic, by Lemma 13 . Hence, $\left(\overline{\mathcal{M}^{c}}, \bar{x}\right) \in \mathcal{E}_{A}$. Then, the proof continues like for the proof of Theorem 2 in order to prove that $\overline{\mathcal{M}^{c}}, \bar{x} \| S$.

Theorem 15. Let $C_{\varphi} \subseteq \operatorname{Con}_{\varphi}-\{\neg\}$. Then, $\operatorname{UL}\left(C_{\varphi}\right)+A$ is sound and strongly complete for the logic $\left(\mathcal{L}\left(\mathbb{P}, C_{\varphi}\right), \mathcal{E}_{A}, \Vdash\right)$.

Proof. Let $\mathrm{C}_{\varphi}^{\prime}:=\mathrm{C}_{\varphi} \cup\{\wedge, \vee\}$. Then, by Lemma 14, $\mathrm{UL}\left(\mathrm{C}_{\varphi}^{\prime}\right)+A$ is sound and strongly complete for the logic $\left(\mathcal{L}\left(\mathbb{P}, \mathrm{C}_{\varphi}^{\prime}\right), \mathcal{E}_{A}, \Vdash\right)$. Then, in particular, for all $\Gamma \subseteq \mathcal{L}\left(\mathbb{P}, \mathrm{C}_{\varphi}\right) \subseteq$ $\mathcal{L}\left(\mathbb{P}, \mathrm{C}_{\varphi}^{\prime}\right)$ and all $\varphi \in \mathcal{L}\left(\mathbb{P}, \mathrm{C}_{\varphi}\right) \subseteq \mathcal{L}\left(\mathbb{P}, \mathrm{C}_{\varphi}^{\prime}\right)$, it holds that $\Gamma \Vdash \varphi$ iff $\Gamma \vdash \varphi$ is provable in $\mathrm{UL}\left(\mathrm{C}_{\varphi}^{\prime}\right)+A$. Now, because $\Gamma$ and $\varphi$ contain only connectives of $\mathrm{C}_{\varphi}$ and because $\operatorname{UL}\left(\mathrm{C}_{\varphi}^{\prime}\right)+A$ enjoys strong cut elimination (Theorem 11 , we have that $\Gamma-\varphi$ is provable in $\mathrm{UL}\left(\mathrm{C}_{\varphi}^{\prime}\right)+A$ iff it is provable in $\operatorname{UL}\left(\mathrm{C}_{\varphi}\right)+A$. So, it holds that $\Gamma \Vdash \varphi$ iff $\Gamma \vdash \varphi$ is provable in $\operatorname{UL}\left(\mathrm{C}_{\varphi}\right)+A$.

Example 4. We list in Figure 9 a list of popular substructural logics. This list is taken from [61, Table 2.4]. By default, $i=3$ in all the structural rules of $A$ and we recall that $\otimes, \supset$ and $\subset$ stand for our $\otimes_{3}, \supset_{1}$ and $C_{2}$ respectively. As we will see in Section 8 , for the minimal and (modal) 


\begin{tabular}{|c|c|c|}
\hline$A$ & $\mathrm{C}_{\varphi}$ & $\operatorname{Logic}\left(\mathcal{L}\left(\mathbb{P}, \mathrm{C}_{\varphi}\right), \mathcal{E}_{A}, \Vdash\right)$ \\
\hline $\mathrm{B} C \mathrm{CW}$ & $\wedge \vee \supset$ & Minimal Logic \\
\hline $\mathrm{B} \mathrm{C} \overline{\mathrm{W}}$ & $\top \perp \wedge \vee \supset$ & Intuitionistic Logic J \\
\hline$\overline{\mathrm{B}} \overline{\mathrm{C}} \overline{\mathrm{W}} \mathrm{K}$ & $\top \perp \wedge \vee \supset \square$ & Modal Intuitionistic Logic \\
\hline $\mathrm{B} \mathrm{B}^{\mathrm{c}}$ & $\otimes \supset \subset$ & Associative Lambek Calculus $L$ \\
\hline $\mathrm{BCW}$ & $\otimes \supset \subset \top \perp \wedge \vee$ & Positive Relevant Logic $\mathrm{R}^{+}$ \\
\hline$B C$ & $\otimes \supset \subset \top \perp \wedge \vee$ & $\begin{array}{c}\text { Positive Multiplicative } \\
\text { Additive Linear Logic MALL }\end{array}$ \\
\hline $\mathrm{BCK}$ & $\otimes \supset \subset \top \perp \wedge \vee$ & Positive Affine Logic BCK \\
\hline$\overline{\mathrm{B}} \overline{\mathrm{C}} \overline{\mathrm{W}}$ & $\otimes \supset \subset \top \perp \wedge \vee$ & Positive Relevant Logic $\mathrm{R}^{+}$ \\
\hline BCWM & $\otimes \supset \subset \top \perp \wedge \vee$ & Positive Relevant Logic with Mingle $\mathrm{RM}^{+}$ \\
\hline
\end{tabular}

Figure 9: Some Substructural Logics (without Truth Constants)

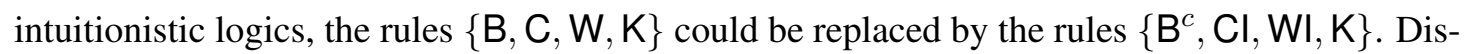
play calculi for some of these logics can be found in [36, 77, 78]. We mention also the modal $\operatorname{logic} \mathrm{S} 4:=\left(\mathcal{L}\left(\mathbb{P}, \mathrm{C}_{\varphi}\right), \mathcal{E}_{A}, \Vdash\right)$, where $\mathrm{C}_{\varphi}:=\{\top, \perp, \wedge, \vee, \square\}$ and $\left.A:=\{\mathrm{T}]\right\}$.

\section{From Update Logic to Dual Update Logic}

In this section we extend the language of update logic with dual connectives. Then, we show that all our results of the previous sections are preserved under this dual extension, including cut admissibility and various sorts of soundness and completeness results.

\subsection{Dual Update Logic}

Notation 4. In the rest of this article, we will use the following dual logical connectives Con $_{\varphi}^{\prime}$ :

$$
\operatorname{Con}_{\varphi}^{\prime}:=\left\{\diamond, \square^{-}, \oplus_{i}, \prec_{i}, \succ_{i} \mid i \in\{1,2,3\}\right\} .
$$

Adding them to the logical connectives $\operatorname{Con}_{\varphi}$, we obtain the full set of logical connectives Con considered in this article:

$$
\text { Con }:=\operatorname{Con}_{\varphi} \cup \operatorname{Con}_{\varphi}^{\prime}
$$

The connectives $\diamond, \square^{-}$are unary connectives and $\oplus_{i}, \prec_{i}, \succ_{i}$ (where $i$ ranges over $\{1,2,3\}$ ) are binary connectives. To save parenthesis, we use the following ranking of binding strength: $\otimes_{i}, \supset_{i}, \subset_{i}, \oplus_{i}, \prec_{i}, \succ_{i}, \wedge, \vee, \rightarrow$ (where $i$ ranges over $\{1,2,3\}$ ).

The triple of connectives $\left(\oplus_{3}, \prec_{1}, \succ_{2}\right)$ corresponds to the usual triple of dual substructural connectives $(\oplus, \prec, \succ)$ of the literature (they are also sometimes denoted $(\oplus, \oslash, \oslash)[51])$. The structural connectives $\operatorname{Struc}(f)$ associated to each $f \in\left\{\oplus_{\mathrm{i}}, \prec_{\mathrm{i}}, \succ_{\mathrm{i}} \mid i \in\{1,2,3\}\right\}$ are defined 


\begin{tabular}{|l|l|l|l|l|l|l|l|}
\hline$\otimes_{\mathrm{i}}$ & $\supset_{\mathrm{i}}$ & $\subset_{\mathrm{i}}$ & $\wedge$ & $\square$ & $\diamond^{-}$ & $\neg$ & $\top$ \\
\hline$\oplus_{\mathrm{i}}$ & $\succ_{\mathrm{i}}$ & $\prec_{\mathrm{i}}$ & $\vee$ & $\diamond$ & $\square^{-}$ & $\neg$ & $\perp$ \\
\hline
\end{tabular}

$$
i \in\{1,2,3\}
$$

Figure 10: Connectives of Con with their Respective Duals

by $\operatorname{Struc}(f):=\left\{,_{\mathrm{i}} \mid i \in\{1,2,3\}\right\}$.

In Figure 10, we present the connectives of Con organized in such a way that each connective is the dual of the connective above or below it.

Definition 20 (Dual update logic). We define the evaluation relation $\models \subseteq \mathcal{E} \times \mathcal{L}$ (P, Con) as follows. Let $(\mathcal{M}, x) \in \mathcal{E}$ be a pointed substructural model and let $\varphi \in \mathcal{L}(\mathbb{P}$, Con). The truth conditions for the connectives of $\operatorname{Con}_{\varphi}$ are defined like in Definition 8 . The truth conditions for the connectives $\oplus_{\mathrm{i}}, \prec_{\mathrm{i}}, \succ_{\mathrm{i}}$ are defined as follows: for all $i \in\{1,2,3\}$, we have that

$$
\begin{aligned}
& \mathcal{M}, x \Vdash \varphi \oplus_{\mathrm{i}} \psi \quad \text { iff } \quad \text { for all } y, z \in \mathcal{P} \text { such that } \sigma_{i}(x, y, z) \in \mathcal{R} \text {, } \\
& \mathcal{M}, y \Vdash \varphi \text { or } \mathcal{M}, z \Vdash \psi ; \\
& \mathcal{M}, x \Vdash \varphi \prec_{\mathrm{i}} \psi \quad \text { iff } \quad \text { there are } y, z \in \mathcal{P} \text { such that } \sigma_{i}(x, y, z) \in \mathcal{R} \text {, } \\
& \mathcal{M}, y \Vdash \varphi \text { and not } \mathcal{M}, z \Vdash \psi ; \\
& \mathcal{M}, x \Vdash \varphi \succ_{\mathrm{i}} \psi \quad \text { iff } \quad \text { there are } y, z \in \mathcal{P} \text { such that } \sigma_{i}(x, y, z) \in \mathcal{R} \text {, } \\
& \mathcal{M}, z \Vdash \psi \text { and not } \mathcal{M}, y \| \varphi \text {. }
\end{aligned}
$$

The truth conditions for the connectives $\diamond, \square^{-}$are defined as follows:

$$
\begin{aligned}
& \mathcal{M}, x \| \diamond \varphi \quad \text { iff } \quad \text { there is } y \in \mathcal{P} \text { such that } x R y \text { and } \mathcal{M}, y \| \varphi \text {; } \\
& \mathcal{M}, x \Vdash \square^{-} \varphi \quad \text { iff } \quad \text { for all } y \in \mathcal{P} \text { such that } y R x, \mathcal{M}, y \| \varphi \text {. }
\end{aligned}
$$

We extend these definitions to the class $\mathcal{F}$ of pointed substructural frames. We define the evaluation relation $\Vdash \subseteq \mathcal{F} \times \mathcal{L}(\mathbb{P}$, Con $)$ as follows. Let $(F, x)$ be a pointed frame and let $\varphi \in \mathcal{L}(\mathbb{P}$, Con $)$. Then, we have that

$$
\begin{aligned}
F, x \Vdash \varphi \quad \text { iff } \quad \text { for all interpretation function } \mathcal{I} \text { such that }(F, \mathcal{I}) \text { satisfies Persistence, } & \\
& (F, \mathcal{I}), x \Vdash \varphi
\end{aligned}
$$

The triple $(\mathcal{L}(\mathbb{P}$, Con $), \mathcal{E}, \Vdash)$ is a logic, called dual update logic.

Our relational interpretation of the dual substructural connectives differs slightly from the interpretation often found in the literature. Indeed, the relational semantics for a language with dual substructural connectives contains in general two distinct ternary relations $\mathcal{R}_{\oplus}$ and $\mathcal{R}_{\otimes}=$ $\mathcal{R}$ and the dual substructural connectives are usually interpreted over the ternary relation $\mathcal{R}_{\oplus}$ $[51,36]$. The interaction between these two ternary relations is then controlled by means of the Grishin rules [38, 51]. In fact, the semantics of our dual connectives as they are defined is such 
that it corresponds in the usual setting to the condition $\mathcal{R}_{\oplus}:=\overline{\mathcal{R}_{\otimes}}$, that is, $\mathcal{R}_{\oplus}$ is the complement of $\mathcal{R}$ in the substructural model. This said, this supplementary constraint on the ternary relations does not enforce the Grishin rules anyway. So, on the one hand, the usual approach enables more flexibility to account for the semantics of the different dual connectives. On the other hand, these connectives may not genuinely be duals of the substructural connectives, as we usually understand duals. In our setting they do correspond to 'genuine' duals, as proved by the following Proposition 16 .

Proposition 16 (Expressiveness). We define inductively the mapping $\tau: \mathcal{L}(\mathbb{P}$, Con $) \rightarrow$ $\mathcal{L}\left(\mathbb{P}, \mathrm{Con}_{\varphi}\right)$ as follows: for all $i \in\{1,2,3\}$, all $\star \in\{*, \bullet\}$, all $f \in$ $\left\{\vee, \wedge, \top, \perp, \otimes_{i}, \supset_{i}, \subset_{i} \mid i \in\{1,2,3\}\right\}$, all $f^{\prime} \in\left\{\square, \diamond^{-}, \neg, \vee\right\}$,

$$
\begin{aligned}
\tau(p) & :=p & \tau(\varphi f \psi) & :=\tau(\varphi) f \tau(\psi) \\
\tau(\diamond \varphi) & :=\neg \square \neg \tau(\varphi) & \tau\left(f^{\prime} \varphi\right) & :=f^{\prime} \tau(\varphi) \\
\tau\left(\square^{-} \varphi\right) & :=\neg \diamond^{-} \neg \tau(\varphi) & \tau(\star X) & :=\star \tau(X) \\
\tau\left(\varphi \oplus_{i} \psi\right) & :=\neg\left(\neg \tau(\varphi) \otimes_{i} \neg \tau(\psi)\right) & \tau\left(X,{ }_{i} Y\right) & :=\tau(X){ }_{i} \tau(Y) \\
\tau\left(\varphi \succ_{i} \psi\right) & :=\neg\left(\neg \tau(\varphi) \supset_{i} \neg \tau(\psi)\right) & & \\
\tau\left(\varphi \prec_{i} \psi\right) & :=\neg\left(\neg \tau(\varphi) \subset_{i} \neg \tau(\psi)\right) & &
\end{aligned}
$$

Then, for all $(\mathcal{M}, x) \in \mathcal{E}$, for all $X \in \mathcal{S}(\mathbb{P}$, Con $)$, we have that

$$
\mathcal{M}, x \|^{A} X \quad \text { iff } \mathcal{M}, x\left\|^{A} \tau(X) \quad \mathcal{M}, x\right\|^{K} X \text { iff } \mathcal{M}, x \|^{K} \tau(X)
$$

Hence, the following hold:

- $(\mathcal{L}(\mathbb{P}$, Con $), \mathcal{E}, \Vdash) \equiv\left(\mathcal{L}\left(\mathbb{P}, \operatorname{Con}_{\varphi}\right), \mathcal{E}, \Vdash\right)$;

- $(\mathcal{L}(\mathbb{P}$, Con $), \mathcal{E}, \Vdash) \equiv\left(\mathcal{S}(\mathbb{P}\right.$, Con $\left.), \mathcal{E}, \|^{A}\right) \equiv\left(\mathcal{S}\left(\mathbb{P}, \operatorname{Con}_{\varphi}\right), \mathcal{E}, \|^{A}\right)$;

- $(\mathcal{C}($ Con $), \mathcal{E}, \Vdash) \equiv\left(\mathcal{C}\left(\operatorname{Con}_{\varphi}\right), \mathcal{E}, \Vdash\right)$.

Proof. The proof of Expression 19 is by an easy induction on $\varphi$.

Proposition 16 shows that the addition of the dual connectives does not add any expressive power to our update logic. So, update logic and dual update logic have the same expressive power.

\subsection{A Display Calculus for Dual Update Logic}

In this section, we provide a display calculus for dual update logic. It extends the display calculus for update logic. The results of this section generalize all the previous results given in this article (except the results about expressiveness).

Definition 21 (Display calculus for $\mathcal{L}\left(\mathbb{P}\right.$, Con)). Let $\mathrm{C}_{\varphi} \subseteq$ Con. The display calculus for $\mathcal{C}\left(C_{\varphi}\right)$, denoted $\mathrm{UL}\left(\mathrm{C}_{\varphi}\right)$, is the display calculus defined in Figures 4,5 and 11 restricted to the logical and structural rules mentioning the connectives of $\mathrm{C}_{\varphi}$ and $\operatorname{Struc}\left(\mathrm{C}_{\varphi}\right)$. If $\mathrm{C}_{\varphi}=$ Con then $\mathrm{UL}\left(\mathrm{C}_{\varphi}\right)$ is denoted $\mathrm{UL}^{\prime}$. For better readability, the brackets for binary connectives are omitted. 
Dual Substructural Connectives:

$\frac{U \vdash \varphi,_{\mathrm{i}} \psi}{U \vdash \varphi \oplus_{\mathrm{i}} \psi} \oplus_{K}^{\mathrm{i}} \quad \frac{\varphi \vdash X \quad \psi \vdash Y}{\varphi \oplus_{\mathrm{i}} \psi \vdash X,_{\mathrm{i}} Y} \oplus_{A}^{\mathrm{i}}$

$\frac{\varphi \vdash X \quad Y \vdash \psi}{* X,_{\mathrm{j}} Y \vdash \varphi \succ_{\mathrm{j}} \psi} \prec_{K}^{\mathrm{j}} \quad \frac{\psi \vdash X,_{\mathrm{i}} \varphi}{\varphi \succ_{\mathrm{j}} \psi \vdash X} \prec_{A}^{\mathrm{j}}$

$\frac{X \vdash \varphi \quad \psi \vdash Y}{X{ }_{{ }_{\mathrm{k}}} * Y \vdash \varphi \prec_{\mathrm{k}} \psi} \succ_{K}^{\mathrm{k}} \quad \frac{\psi \vdash \varphi,_{\mathrm{i}} X}{\varphi \prec_{\mathrm{k}} \psi \vdash X} \succ_{A}^{\mathrm{k}}$

$(i, j, k) \in\{(1,2,3),(2,3,1),(3,1,2)\}$

Dual Modal Connectives:

$$
\begin{array}{ll}
\frac{U \vdash * \bullet * \varphi}{U \vdash \square^{-} \varphi} \square_{K}^{-} & \frac{\varphi \vdash X}{\square^{-} \varphi \vdash * \bullet * X} \square_{A}^{-} \\
\frac{X \vdash \varphi}{* \bullet * X \vdash \diamond \varphi} \diamond_{K} & \frac{* \bullet * \varphi \vdash U}{\diamond \varphi \vdash U} \diamond_{A}
\end{array}
$$

Figure 11: Logical Rules for Dual Connectives 


\begin{tabular}{|c|c|c|c|}
\hline Connective $f$ & Trace of $f$ & $S(f, \varphi, \chi, \psi)$ & Display rule \\
\hline$\oplus_{\mathrm{i}}$ & $(+,+) \mapsto+$ & $\psi \Vdash \varphi \oplus_{\mathrm{i}} \chi$ & $Z \Vdash X{ }_{\mathrm{i}} Y$ \\
$\succ_{\mathrm{j}}$ & $(+,-) \mapsto-$ & $\chi \succ_{\mathrm{j}} \psi \| \varphi$ & $* Y,{ }_{\mathrm{j}} Z \Vdash X$ \\
$\prec_{\mathrm{k}}$ & $(-,+) \mapsto-$ & $\psi \prec_{\mathrm{k}} \varphi \Vdash \chi$ & $Z{ }_{{ }_{\mathrm{k}}} * X \Vdash Y$ \\
\hline
\end{tabular}

$$
(i, j, k) \in\{(1,2,3),(2,3,1),(3,1,2)\}
$$

Figure 12: Transforming a Founded Family into Display Rules

Connection with Goré's display logic. Goré [36] defined display calculi for substructural logics as well. His dual substructural connectives $\{\oplus, \prec, \succ\}$ are defined proof-theoretically by means of the same introduction rules as ours (given in Figure 11) modulo the translation given on Page 22. However, because his display rules are different, the semantics of his dual connectives is also different (even if it follows the usual semantics found in the literature [23]). As we already explained in Section 4.2, our first two sets of display rules generalize Goré's display rules, but at the same time impose more constraints on the logic because they are both bound by the structural negation $*$, which somehow ties together the two sets of display rules (like in Wansing's display calculus for modal logic). This binding is expressed at the semantic level by the fact that $\mathcal{R}_{\oplus}$ is the complement of $\mathcal{R}_{\otimes}=\mathcal{R}$, whereas the definition of $\mathcal{R}_{\oplus}$ is usually independent from the definition of $\mathcal{R}_{\otimes}$, but might be related to it by adding (some of) the Grishin rules to the calculus (see [36, Sect. 6.2] and [51]). This extra flexibility of Goré's display calculus allows him to keep two versions of the structural rules, depending on whether or not the principal structures are antecedent or consequent parts. Thus, he has right and left versions of \{commutativity, weakening, contraction, associativity $\}$. Due to the binding effect of the structural negation $*$, we can prove with our display rules that right and left \{commutativity, weakening, contraction collapse, but not necessarily right and left associativity. This implies that we cannot capture semi-commutative relevance logic as Gore does. On the other hand, our dual update logic is close to the usual semantics given to dual connectives, as demonstrated by our Proposition 16.

A similar phenomenon occurs in Goré's display calculus for modalities and their duals. He obtains two distinct pairs of residuated modalities $\left(\diamond^{-}, \square\right)$ and $\left(\diamond, \square^{-}\right)$, but there is no 'dual' connection between $\diamond$ and $\square$ and between $\diamond^{-}$and $\square^{-}$like in Proposition 16, via $\neg$. Again, this is due to the fact that there is no structural connectives $*$ in his display calculus to bind the two pairs of residuals. So, in that case, one has to admit that his restricted form of the display rules seems to be problematic.

Connection with Dunn's Gaggle Theory. We can extend our connection to Gaggle Theory for the dual connectives. If $\mathrm{C}_{\varphi}:=\left\{\top, \perp, \oplus_{\mathrm{i}}, \prec_{\mathrm{j}}, \succ_{\mathrm{k}}\right\}$ for some $(i, j, k) \in\{(1,2,3),(2,3,1),(3,1,2)\}$, then the triple of connectives $O P_{i}:=\left\{\oplus_{\mathrm{i}}, \prec_{\mathrm{j}}, \succ_{\mathrm{k}}\right\}$ is a founded family of the partial gaggle $\mathcal{T}:=\left(\mathcal{L}\left(\mathbb{P}, \mathrm{C}_{\varphi}\right), \Vdash, O P_{i}\right)$ and $\oplus_{\mathrm{i}}$ is its head. As we explained in Section 4.2, our structural connectives are associated to each founded family of a partial gaggle, and not to pairs of residuated logical connectives (a founded family might 


\begin{tabular}{|c|c|c|c|c|c|cc|c|}
\hline Founded family (head first) & $\neg$ & $\wedge$ & $\vee$ & $\square \diamond^{-}$ & $\diamond \square^{-}$ & $\otimes_{\mathrm{i}} \supset_{\mathrm{j}} \subset_{\mathrm{k}}$ & $\oplus_{\mathrm{i}} \prec_{\mathrm{j}} \succ_{\mathrm{k}}$ \\
\hline Structural connective & $*$ &, &, & $\bullet$ & $\bullet$ & $\mathrm{r}_{\mathrm{i}}$ & $\mathrm{\gamma}_{\mathrm{i}}$ \\
\hline
\end{tabular}

$$
(i, j, k) \in\{(1,2,3),(2,3,1),(3,1,2)\}
$$

Figure 13: Families of Connectives

contain several residuated pairs). Following this idea, we obtain the second set of display rules from the dual substructural connectives. This transformation from the 'dual' founded family to the second set of display rules is given in the last three rows of Figure 12. Hence, we can obtain all our display rules from Gaggle Theory.

We summarize the different families of connectives we obtain in Figure 13 . Each family corresponds to a founded family of a partial gaggle and is associated with the structural connective of the head of this family. Sometimes two families of connectives are associated with the same structural connectives. In that case it means that these two families are dual of each other and the semantics of the associated structural connective should be interpreted with respect to $\|^{A}$ for the first family and with respect to $\|^{K}$ for the second family.

\subsubsection{Admissibility of the Cut Rule}

The following Theorem 17 summarizes and generalizes all our results about cut admissibility in this article. In fact, Theorems 4 and 11 are corollaries of Theorem 17.

Theorem 17 (Strong cut elimination). The display calculus $U L^{\prime}+A$ is a proper display calculus. Hence, $U L^{\prime}+$ A enjoys strong cut-elimination and therefore the cut rule is an admissible rule of $U L^{\prime}+A$.

Proof. The logical rules for the dual substructural connectives can be translated into the corresponding logical rules of [36] (for some of them, the other ones being completely symmetric). As in [36], we can easily show that they satisfy condition $(C 8)$. As for the modal rules, condition $(C 8)$ has already been proven [78]. Therefore, $\mathrm{UL}^{\prime}$ is a proper display calculus. It enjoys strong cut-elimination because of [78, Theorem 23].

\subsubsection{Soundness, Completeness and Conservativity}

The following Theorems 18 and 19 summarize and generalize all our soundness and completeness results in this article. In fact, Theorem 2 and Corollary 1 are instances of Theorem 18 and Theorem 15 is an instance of Theorem 19 .

Theorem 18 (Soundness and strong completeness). Let $C_{\varphi} \subseteq$ Con. The display calculus $U L\left(C_{\varphi}\right)$ is sound and strongly complete for the logic $\left(\mathcal{C}\left(C_{\varphi}\right), \mathcal{E}, \Vdash\right)$. 
Proof. The soundness is proved without any particular difficulty. For completeness, let $\Gamma \subseteq$ $\mathcal{C}\left(\mathrm{C}_{\varphi}\right)$ and let $S:=X \vdash Y \in \mathcal{C}\left(\mathrm{C}_{\varphi}\right)$, and assume that $\Gamma \Vdash S$. We must prove that $\Gamma \vdash S$ is provable in $\mathrm{UL}\left(\mathrm{C}_{\varphi}\right)$.

By Proposition 16 , we have that $\tau(\Gamma) \Vdash \tau(S)$, where $\tau(\Gamma):=$ $\{\tau(U) \vdash \tau(V) \mid U \vdash V \in \Gamma\} \subseteq \mathcal{C}\left(\operatorname{Con}_{\varphi}\right)$ and $\tau(S):=\tau(X) \vdash \tau(V) \in \mathcal{C}\left(\operatorname{Con}_{\varphi}\right)$. Therefore, by strong completeness of UL (Theorem 2), we have that $\tau(\Gamma) \vdash \tau(S)$ is provable in UL $(*)$.

Now, one can prove by induction that for all $U, V \in \mathcal{S}\left(\mathbb{P}, \mathrm{C}_{\varphi}\right)$, the following are provable in $U L\left(\mathrm{C}_{\varphi} \cup \operatorname{Con}_{\varphi}\right)$ :

$$
\tau_{2}(\tau(V)) \vdash V \quad U \vdash \tau_{1}(\tau(U)) .
$$

(The mappings $\tau_{1}$ and $\tau_{2}$ are defined in Proposition 1.) The only tricky cases are when $V:=$ $\varphi \oplus_{\mathrm{i}} \psi, \varphi \succ_{\mathrm{i}} \psi$ or $\varphi \prec_{\mathrm{i}} \psi$. We prove these three cases below:

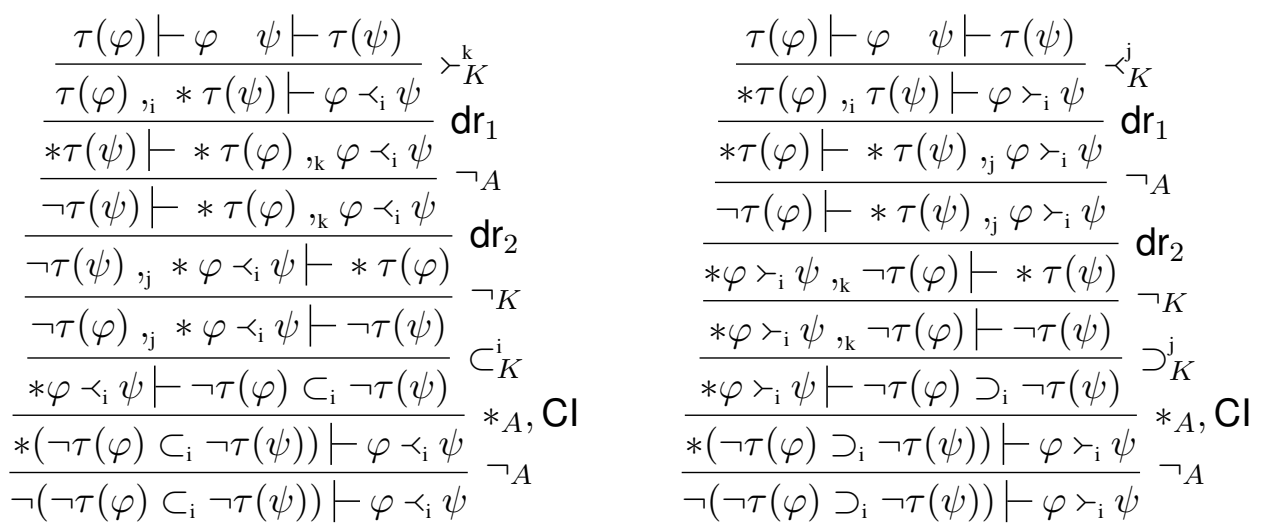

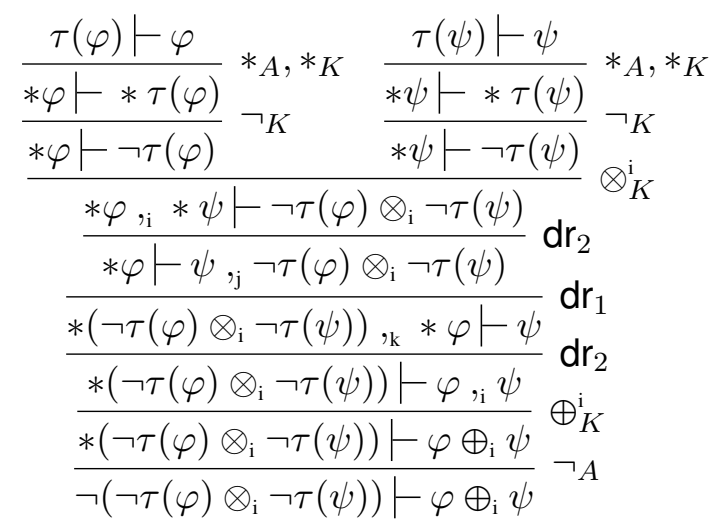

Moreover, by Expression (9), we have that $\tau(U) \vdash \tau(V)$ is provable in UL iff $\tau_{1}(\tau(U)) \vdash \tau_{2}(\tau(V))$ is provable in UL. So, by Expression (20) and by admissibility of the cut rule in UL (because of Theorem 17, we have that $\tau(U) \vdash \tau(V)$ is provable in UL iff $U \vdash V$ is provable in $\mathrm{UL}\left(\mathrm{C}_{\varphi} \cup \mathrm{Con}_{\varphi}\right)$. But $U, V \in \mathcal{S}\left(\mathbb{P}, \mathrm{C}_{\varphi}\right)$, so, in fact, $U \vdash V$ is provable in $\mathrm{UL}\left(\mathrm{C}_{\varphi} \cup \operatorname{Con}_{\varphi}\right)$ iff $U \vdash V$ is provable in $\mathrm{UL}\left(\mathrm{C}_{\varphi}\right)$, because $\mathrm{UL}$ enjoys strong cut-elimination. 
So, finally, we have that $\tau(U) \vdash \tau(V)$ is provable in $\mathrm{UL}$ iff $U \vdash V$ is provable in $\mathrm{UL}\left(\mathrm{C}_{\varphi}\right)$, for all $U, V \in \mathcal{S}\left(\mathbb{P}, \mathrm{C}_{\varphi}\right)$. Thus, $\tau(\Gamma) \vdash \tau(S)$ is provable in $\mathrm{UL}$ iff $\Gamma \vdash S$ is provable in $\mathrm{UL}\left(\mathrm{C}_{\varphi}\right)$ $(* *)$. Combining $(*)$ and $(* *)$, we obtain that $\Gamma \vdash S$ is provable in $\mathrm{UL}\left(\mathrm{C}_{\varphi}\right)$.

Here is our second main theorem.

Theorem 19 (Soundness and strong completeness). Let $C_{\varphi} \subseteq$ Con $-\{\neg\}$. Then, the display calculus $U L\left(C_{\varphi}\right)+A$ is sound and strongly complete for the logic $\left(\mathcal{L}\left(\mathbb{P}, C_{\varphi}\right), \mathcal{E}_{A}, \Vdash\right)$.

Proof. The proof of soundness follows from Theorem 15. So, we only prove the strong completeness. Let $\mathrm{L}:=\left(\mathcal{L}\left(\mathbb{P}, \mathrm{C}_{\varphi}\right), \mathcal{E}_{A}, \Vdash\right)$ and let $\Gamma \subseteq \mathcal{L}\left(\mathbb{P}, \mathrm{C}_{\varphi}\right)$ and $\varphi \in \mathcal{L}\left(\mathbb{P}, \mathrm{C}_{\varphi}\right)$. The proof follows the same lines as the proofs of Lemma 14 and Theorem 15 , so we do not spell it out as we did it there. Assume that $\Gamma \Vdash \varphi$ and assume towards a contradiction that $\tau(\Gamma) \vdash \tau(\varphi)$ is not provable in $\mathrm{UL}+A$. This means in other words that $\{\tau(\Gamma), * \tau(\varphi)\}$ is $\mathrm{UL}+A$-consistent. Therefore, in the canonical substructural model $\mathcal{M}^{c}$ associated to $\mathrm{UL}+A$, there is $x \in \mathcal{M}^{c}$ such that $\mathcal{M}^{c}, x \Vdash\{\tau(\Gamma), * \tau(\varphi)\}$. The validity of Expression (18) can be extended to the language with the Boolean negation $\neg$ and we can therefore prove that for all $\varphi \in \mathcal{L}\left(\mathbb{P}, \mathrm{C}_{\varphi}\right)$, we have that $\overline{\mathcal{M}^{c}}, \bar{x} \Vdash \tau(\varphi)$ iff $\mathcal{M}^{c}, x \| \tau(\varphi)$, because $\tau(\varphi) \in \mathcal{L}\left(\mathbb{P}, \operatorname{Con}_{\varphi}\right)$. Moreover, by Lemma 13 and [61, Corollary 11.36], $\left(\overline{\mathcal{M}^{c}}, \bar{x}\right) \in \mathcal{E}_{A}$. So, there is $(\mathcal{M}, x) \in \mathcal{E}_{A}\left(\right.$ take $\left(\overline{\mathcal{M}^{c}}, \bar{x}\right)$ ) such that $\mathcal{M}, x \Vdash\{\tau(\Gamma), * \tau(\varphi)\}$. This means that it is not the case that $\tau(\Gamma) \Vdash \tau(\varphi)$. Then, by Proposition 16, this entails that it is not the case that $\Gamma \Vdash \varphi$. This is impossible by assumption. Therefore, $\tau(\Gamma) \vdash \tau(\varphi)$ is provable in $\mathrm{UL}+A$. Then, from Expression $(* *)$ in the proof of Theorem 18, we derive that $\tau(\Gamma) \vdash \tau(\varphi)$ is provable in $\mathrm{UL}+A$ iff $\Gamma \vdash \varphi$ is provable in $\mathrm{UL}\left(\mathrm{C}_{\varphi}\right)+A$. Hence, this entails that $\Gamma \vdash \varphi$ is provable in $\mathrm{UL}\left(\mathrm{C}_{\varphi}\right)+A$.

Because our display calculi enjoy strong cut elimination, we immediately have the following property, which generalizes [61, Theorem 11.52].

Corollary 2 (Conservativity). Let $C_{\varphi}^{\prime} \subseteq C_{\varphi} \subseteq$ Con $-\{\neg\}$. The logic $\left(\mathcal{L}\left(\mathbb{P}, C_{\varphi}\right), \mathcal{E}_{A}, \Vdash\right)$ is a conservative extension of the logic $\left(\mathcal{L}\left(\mathbb{P}, C_{\varphi}^{\prime}\right), \mathcal{E}_{A}, \Vdash\right)$.

Proof. The proof is standard. See for example [54] for details.

\section{Case Study: Intuitionistic Logic}

In this section, we focus on intuitionistic logic and we will apply our previous results to obtain display calculi. Some display calculi have already been proposed in the literature [78, 36, 37 , 79], sometimes with modal and tense extensions [78].

Notation 5. In this section, we will use the following set of logical connectives:

$$
\text { Con }_{l}:=\{\top, \perp, \wedge, \vee, \supset\}
$$

where $\supset$ is the connective $\supset_{1}(\supset$ can also stand for $\Rightarrow)$. 


\subsection{Intuitionistic Logic}

We present the Kripke-style semantics of intuitionistic logic.

Definition 22 (Intuitionistic Kripke model). An intuitionistic Kripke frame is a pair $(W, \sqsubseteq)$ where $W$ is a non-empty set (of possible worlds) and $\sqsubseteq \subseteq W \times W$ is a reflexive and transitive binary relation over $W$. An intuitionistic Kripke model is a triple $M:=(W, \sqsubseteq, V)$ where $(W, \sqsubseteq)$ is an intuitionistic Kripke frame and $V: \mathbb{P} \rightarrow 2^{W}$ is a mapping obeying the persistence condition: for all $x, y \in W$, for all $p \in \mathbb{P}$,

$$
\text { if } x \in V(p) \text { and } x \sqsubseteq y \text { then } y \in V(p) .
$$

(Persistence)

We abusively write $x \in M$ for $x \in W$ and $(M, x)$ is called a pointed intuitionistic Kripke model. The class of all pointed intuitionistic Kripke models (frames) is denoted $\mathcal{K}_{\text {Int }}$ (resp. $\left.\mathcal{F}_{\text {Int }}\right)$.

Note that the above Persistence condition corresponds to the Persistence condition of Definition 4 for substructural models.

Definition 23 (Intuitionistic logic). The intuitionistic evaluation relation $\models \subseteq \mathcal{K}_{\text {Int }} \times$ $\mathcal{L}\left(\mathbb{P}\right.$, Con $\left._{\mathbf{I}}\right)$ is defined inductively as follows. Let $(M, x)$ be a pointed intuitionistic Kripke model and $\varphi \in \mathcal{L}\left(\mathbb{P}, \mathrm{Con}_{\mathrm{I}}\right)$. The truth conditions for the truth constants $T, \perp$ and the propositional connectives $\wedge, \vee$ are defined like in Definition 8 , The truth condition for the connective $\supset$ is defined as follows:

$$
M, x \models \chi \supset \psi \quad \text { iff } \quad \text { for all } y \in M \text { such that } x \sqsubseteq y, \text { if } M, y \models \chi \text { then } M, y \models \psi
$$

Hence, $\left(\mathcal{L}\left(\mathbb{P}\right.\right.$, Con $\left.\left._{\mathbf{I}}\right), \mathcal{K}_{\text {Int }}, \models\right)$ is a logic, called intuitionistic logic.

\subsection{A Display Calculus for Intuitionistic Logic}

Kripke [41] suggested the following 'informational' reading of intuitionistic Kripke frames: $W$ is a set of information states and $\sqsubseteq$ is the relation of possible expansion of information states over time. Under this interpretation, intuitionistic logic may then be viewed as the logic of cumulative research [39]. This informational and dynamic reading of intuitionistic logic has also been stressed and discussed to a large extent by van Benthem [72, 52]. We will show in this section that the intuitionistic substructural models of Definition 24 below are in fact equivalent to intuitionistic Kripke models. Intuitionistic substructural models connect the possible expansion relation $\sqsubseteq$ with our ternary relation $\mathcal{R}$ by means of Expression 21 . Given this formal connection, Kripke's informational reading of $\sqsubseteq$ is completely coherent with our dynamic interpretation of the ternary relation $\mathcal{R}$ (spelled out in Section 2.2).

Definition 24 (Intuitionistic substructural model). An intuitionistic substructural model is a substructural model $\mathcal{M}$ whose accessibility relations are plump and such that for all $x, y, z \in \mathcal{M}$, the following holds:

$$
\mathcal{R} x y z \text { iff } x \sqsubseteq z \text { and } y \sqsubseteq z
$$

The class of all pointed intuitionistic substructural models is denoted $\mathcal{E}_{\mathrm{Int}}$. 
Now, we prove by a series of Lemmata that intuitionistic substructural models can be equivalently represented by intuitionistic Kripke models (Lemma 22).

Lemma 20. Let $C_{\varphi} \subseteq$ Con $-\{\neg\}$. Then, $\operatorname{UL}\left(C_{\varphi}\right)+\left\{\left|B^{c} C / W\right| K\right\}$ is sound and strongly complete for the logic $\left(\mathcal{L}\left(\mathbb{P}, C_{\varphi}\right), \mathcal{E}_{\text {Int }}, \Vdash\right)$.

Proof. By application of Theorem $19, \mathrm{UL}\left(\mathrm{C}_{\varphi}\right)+\left\{\overline{\left.\mathrm{B}^{c}|\mathrm{Cl}| \mathrm{WI} \mid \mathrm{K}\right\}}\right\}$ is sound and strongly complete for the logic $\left(\mathcal{L}\left(\mathbb{P}, \mathrm{C}_{\varphi}\right), \mathcal{E}_{\text {Int }}, \Vdash\right)$, where $\mathcal{E}_{\text {Int }}$ is the class of pointed substructural models $(\mathcal{M}, x)$ whose accessibility relations are plump and such that the following hold: for all $x, y, z \in \mathcal{M}$,

if $\exists u(\mathcal{R} y z u \wedge \mathcal{R} x u w)$ then $\exists u(\mathcal{R} x y u \wedge \mathcal{R} u z w)$
$\mathcal{R} x y z$ iff $\mathcal{R} y x z$
$\mathcal{R} x x x$
if $\mathcal{R} x y z$ then $y \sqsubseteq z$

So, to prove our result, we only need to show that these statements are equivalent to Expression 21] when accessibility relations are plump.

First, we are going to prove that if $\mathcal{R}$ satisfies Expressions $\left\{\left|\mathrm{B}^{c} \mathrm{Cl}\right| \mathrm{W} \mid \mathrm{K}\right\}$, then Expression 21) holds. Assume that Expressions $\left\{\overline{\left.B^{c}|C||W| K\right\}}\right.$ hold, we are going to prove that the left to right implication of Expression (21) holds. Let $x, y, z \in \mathcal{M}$ and assume that $\mathcal{R} x y z$ holds. Then, by Expression $[\mathbb{K} \mid$, we have that $y \sqsubseteq z$. Moreover, by Expression $(\mathbb{C l})$, we also have that $\mathcal{R} y x z$. So, again by Expression $(\mathbb{K})$, we have that $x \sqsubseteq z$. This proves the left to right implication of Expression 21]. Now, we prove the right to left implication of Expression [21]. Assume that $x \sqsubseteq z$ and $y \sqsubseteq z$. Then, by Expression (WI), we have that $\mathcal{R} z z z$. Then, because $\mathcal{R}$ is plump and $x \sqsubseteq z$ and $y \sqsubseteq z$, we have that $\mathcal{R} x y z$. This proves the second implication of Expression 21]. So, we have proved Expression 21.

Second, we prove the other direction, i.e., if $\mathcal{R}$ satisfies Expression (21), then Expressions $\left\{\mathrm{B}^{c}|\mathrm{Cl}| \mathrm{WI} \mid \mathrm{K}\right\}$ hold. We start to prove Expression (W/ $)$. Because $x \sqsubseteq x$, then, by Expression (21), we have that $\mathcal{R} x x x$. We prove Expression (Cl). If $\mathcal{R} x y z$, then $x \sqsubseteq z$ and $y \sqsubseteq z$ by Expression (21). Then, $y \sqsubseteq z$ and $x \sqsubseteq z$. So, again by Expression (21) (in the other direction), we have that $\mathcal{R} y x z$. We prove Expression $\left(\overline{\mathrm{B}^{\mathrm{C}}}\right)$. If $\mathcal{R} x(y z) w$, then there is $u \in \mathcal{M}$ such that $(\mathcal{R} y z u \wedge \mathcal{R} x u w)$. So, by Expression $(21)$, we have $y \sqsubseteq u, z \sqsubseteq u, x \sqsubseteq w$ and $u \sqsubseteq w$. Then, by reflexivity and transitivity of $\sqsubseteq$, we have $(y \sqsubseteq w, x \sqsubseteq w)$ and $(z \sqsubseteq w, w \sqsubseteq w)$. Therefore, again by Expression (21), we have that $\mathcal{R} x y w$ and $\mathcal{R} w z w$. Hence, there is $u \in \mathcal{M}$ (take $u=w$ ) such that $(\mathcal{R} x y u$ and $\mathcal{R} u z w)$. That is, $\mathcal{R}(x y) z w$. The proof of Expression $(\mathbb{K})$ follows straightforwardly from Expression 21.

Lemma 21 (Persistence Lemma). Let $C_{\varphi} \subseteq \operatorname{Con}_{\varphi}-\{\neg\}$ and let $(\mathcal{M}, x) \in \mathcal{E}_{\text {Int. Then, for all }}$ $y \in \mathcal{M}$, for all $\varphi \in \mathcal{L}\left(\mathbb{P}, C_{\varphi}\right)$, the following holds:

$$
\text { if } \mathcal{M}, x \Vdash \varphi \text { and } x \sqsubseteq y \text { then } \mathcal{M}, y \| \varphi
$$

(Persistence')

Moreover, for all $\varphi, \psi \in \mathcal{L}\left(\mathbb{P}, C_{\varphi}\right)$, the following also holds:

$$
\mathcal{M}, x \Vdash \varphi \Rightarrow \psi \text { iff } \mathcal{M}, x \Vdash \varphi \supset \psi
$$


(We recall that $\Rightarrow$ is the intuitionistic implication, whose truth condition is given in Definition 4)

Proof. The proof is standard and is by induction on the formula $\varphi$. The basic case $\varphi:=p$ holds because of condition (Persistence) of Definition 4 Expression (22) follows from Expressions (21) and (Persistence') and the definitions of the truth conditions of $\supset=\supset_{1}$ and $\Rightarrow$.

Note that Expression 22] shows that the intuitionistic implication $\Rightarrow$ can be 'simulated' by the implication $\supset$ in the intuitionistic version of the substructural framework. So, if we are only interested in intuitionistic logics and not in subintuitionistic logics, then we can refrain from using and introducing the inclusion relation $\sqsubseteq$ in the substructural semantics and consider only a ternary relation satisfying the conditions $\left\{\left\langle\mathrm{B}^{c}|\mathrm{Cl}| \mathrm{WI}\right| \mathrm{K}\right\}$ and a specific formulation of the Persistence condition.

Lemma 22. Every pointed intuitionistic substructural model $(\mathcal{M}, x) \in \mathcal{E}_{\text {Int }}$ can be mapped to a pointed intuitionistic Kripke model $(M, x) \in \mathcal{K}_{\text {Int }}$ such that for all $\varphi \in \mathcal{L}\left(\mathbb{P}, \operatorname{Con}_{l}\right), \mathcal{M}, x \Vdash \varphi$ if, and only if, $M, x \models \varphi(*)$. The converse holds as well.

Proof. Let $(\mathcal{M}, x)=((P, \sqsubseteq), \mathcal{R}, \mathcal{I}, x) \in \mathcal{E}_{\text {Int }}$ be a pointed intuitionistic substructural model. We define the pointed intuitionistic Kripke model $(M, x) \in \mathcal{K}_{\text {Int }}$ associated to $(\mathcal{M}, x)$ as follows: $(M, x):=(P, \sqsubseteq, V, x)$, where for all $y \in P$ and all $p \in \mathbb{P}$, we set $y \in V(p)$ iff $p \in \mathcal{I}(y)$. Then, by Lemma 21, we have that condition (*) holds. Conversely, let $(M, x)=(P, \sqsubseteq, V, x) \in$ $\mathcal{K}_{\text {Int }}$ be a pointed intuitionistic Kripke model. Since $\sqsubseteq$ is not necessarily antisymmetric, we unravel $(M, x)$ to obtain another pointed intuitionistic Kripke model $\left(M^{\prime}, x\right)=\left(W^{\prime}, \sqsubseteq^{\prime}, V^{\prime}, x\right)$ whose relation $\sqsubseteq^{\prime}$ is antisymmetric (and therefore a partial order), like in [18, Definition 4.21]. Then, by [18, Lemma 4.53] and [18, Proposition 2.14], we have that for all $\varphi \in \mathcal{L}\left(\mathbb{P}\right.$, Con $\left._{1}\right)$, $M, x \models \varphi$ iff $M^{\prime}, x \models \varphi$. Then, we define the pointed intuitionistic substructural model $(\mathcal{M}, x) \in \mathcal{E}_{\text {Int }}$ associated to $(M, x)$ as follows: $(\mathcal{M}, x):=\left(\left(W^{\prime}, \sqsubseteq^{\prime}\right), \mathcal{R}, V^{\prime}, x\right)$, where $\mathcal{R}$ is defined by Expression (21) from the partial order $\complement^{\prime}$, and for all $y \in W^{\prime}$ and all $p \in \mathbb{P}$ we set $p \in \mathcal{I}(y)$ iff $y \in V^{\prime}(p)$. Then, by Lemma 21, we can again easily prove that condition $(*)$ holds.

From Lemmata 20 and 22, we obtain our main result for intuitionistic logic:

Theorem 23 (Soundness and strong completeness). The display calculus UL(Con $)+$ $\left\{B^{c}|C|,|W|\right\} \mid$ is sound and strongly complete for intuitionistic logic $\left(\mathcal{L}\left(\mathbb{P}, C_{l} n_{l}\right), \mathcal{K}_{\text {Int }}, \models\right)$.

Proof. The proof follows straightforwardly from Lemmata 20 and 22 .

Lemmata 20 and 22 could give us display calculi which are complete for a wide variety of other intuitionistic logics, including not only the 'standard' modal and tense extensions of intuitionistic logics [78] but also some other logics including some 'non-standard' connectives stemming from our various (substructural) connectives $\operatorname{Con}_{\varphi}$. We leave the investigation of these 'non-standard' logics for future work. 


\section{Conclusion}

We have generalized the framework of substructural logics in several directions. We developed not only a rich family of substructural connectives which are interrelated by means of cyclic permutations, but also extended the language with dual connectives. We provided proper display calculi for all our substructural logics and proved their soundness and strong completeness by means of a Henkin-style construction. We showed that we can obtain our display rules using Dunn's Gaggle Theory and compared our display calculus with the display calculi of Wansing and Goré. In particular, we showed that our display calculus generalizes Wansing's display calculus for modal logic. Then, based on correspondence results of substructural logics, we were able to propose novel display calculi for a very wide range of substructural and non-classical logics. Finally, we focused on intuitionistic logic for which we also provided a display calculus.

In the companion article [7] (which extends and improves [5]) we elicit a number of inference rules that characterize the product update of Dynamic Epistemic Logic (DEL). Outside the DEL paradigm, other kinds of model changing operations have recently been investigated in various kinds of dynamic logics [40, 8, 35, 1, 2, 26, 10, 9]. A natural and interesting line of research would be to investigate whether and how these dynamic logics can be embedded into the substructural framework. Finally, as we mentioned in the introduction, ternary relations could also represent the various revisions, updates and even causal connectives which have been studied in the logics of "common sense reasoning" in artificial intelligence and philosophical logic. Some connections between causal conditionals [33] and ternary semantics have in fact already started to emerge [19]. Other connections with belief revision and conditional logic can be found in [6].

Acknowledgments I thank Olivier Roy and Ole Hjortland for inviting me to the Munich Meeting on Sub-structural Epistemic Logic (January 2013), which was very inspiring. I also thank Marta Bilkova for discussions. I thank Sean Sedwards for checking the English of this article. Finally, I thank the editors of the special issue and Jane Spurr for their patience.

\section{References}

[1] Carlos Areces, Raul Fervari, and Guillaume Hoffmann. Swap logic. Logic Journal of IGPL, 22(2):309-332, 2014.

[2] Carlos Areces, Raul Fervari, and Guillaume Hoffmann. Relation-changing modal operators. Logic Journal of the IGPL, 23(4):601-627, 2015.

[3] Guillaume Aucher. A combined system for update logic and belief revision. In Mike Barley and Nikola K. Kasabov, editors, PRIMA, volume 3371 of Lecture Notes in Computer Science, pages 1-17. Springer, 2004.

[4] Guillaume Aucher. Private announcement and belief expansion: an internal perspective. $J$. Log. Comput., 22(3):451-479, 2012. 
[5] Guillaume Aucher. DEL as a substructural logic. In Alexandru Baltag and Sonja Smets, editors, Outstanding Contributions: Johan F. A. K. van Benthem on Logical and Informational Dynamics, Trends in Logic. Springer, 2014.

[6] Guillaume Aucher. When conditional logic and belief revision meet substructural logics. In Richard Booth, Giovanni Casini, Szymon Klarman, Gilles Richard, and Ivan José Varzinczak, editors, Proceedings of the International Workshop on Defeasible and Ampliative Reasoning, DARe 2015, co-located with the 24th International Joint Conference on Artificial Intelligence (IJCAI 2015), Buenos Aires, Argentina, July 27, 2015., volume 1423 of CEUR Workshop Proceedings. CEUR-WS.org, 2015.

[7] Guillaume Aucher. Dynamic epistemic logic in update logic. Journal of Logic and Computation, Special Issue on Substructural Logics and Information Dynamics, 2016.

[8] Guillaume Aucher, Philippe Balbiani, Luis Fariñas Del Cerro, and Andreas Herzig. Global and local graph modifiers. In Methods for Modalities 5 (M4M-5), Cachan, France, 2007. ENTCS, Elsevier.

[9] Philippe Balbiani, Rachid Echahed, and Andreas Herzig. A dynamic logic for termgraph rewriting. In Hartmut Ehrig, Arend Rensink, Grzegorz Rozenberg, and Andy Schürr, editors, ICGT, volume 6372 of Lecture Notes in Computer Science, pages 59-74. Springer, 2010.

[10] Philippe Balbiani, Andreas Herzig, and Nicolas Troquard. Dynamic logic of propositional assignments: A well-behaved variant of PDL. In LICS, pages 143-152. IEEE Computer Society, 2013.

[11] Alexandru Baltag and Sonja Smets. Conditional doxastic models: A qualitative approach to dynamic belief revision. Electronic Notes in Theoretical Computer Science, 165:5-21, 2006.

[12] Alexandru Baltag and Sonja Smets. The logic of conditional doxastic actions. In R. van Rooij and K. Apt, editors, Special Issue on New Perspectives on Games and Interaction, volume 4 of Texts in Logic and Games, pages 9-31. Amsterdam University Press, 2008.

[13] Alexandru Baltag and Sonja Smets. A qualitative theory of dynamic interactive belief revision. In M. Woolridge G. Bonanno, W. van der Hoek, editor, Texts in Logic and Games, volume 3, pages 9-58. Amsterdam University Press, 2008.

[14] John Barwise and John Perry. Situations and Attitudes. Cambridge, Massachusetts. MIT Press, 1983.

[15] Jon Barwise. Constraints, channels, and the flow of information. Situation theory and its applications, 3:3-27, 1993.

[16] Jc Beall, Ross Brady, J. Michael Dunn, AP Hazen, Edwin Mares, Robert K Meyer, Graham Priest, Greg Restall, David Ripley, John Slaney, et al. On the ternary relation and conditionality. Journal of philosophical logic, 41(3):595-612, 2012. 
[17] Jr. Belnap, Nuel D. Display logic. Journal of Philosophical Logic, 11(4):375-417, 1982.

[18] Patrick Blackburn, Maarten de Rijke, and Yde Venema. Modal Logic, volume 53 of Cambridge Tracts in Computer Science. Cambridge University Press, 2001.

[19] Alexander Bochman. Dynamic causal calculus. In Chitta Baral, Giuseppe De Giacomo, and Thomas Eiter, editors, Principles of Knowledge Representation and Reasoning: Proceedings of the Fourteenth International Conference, KR 2014, Vienna, Austria, July 20-24, 2014. AAAI Press, 2014.

[20] Piero Andrea Bonatti and Nicola Olivetti. Sequent calculi for propositional nonmonotonic logics. ACM Trans. Comput. Logic, 3(2):226-278, April 2002.

[21] John P Burgess. Quick completeness proofs for some logics of conditionals. Notre Dame Journal of Formal Logic, 22(1):76-84, 1981.

[22] Agata Ciabattoni, Revantha Ramanayake, and Heinrich Wansing. Hypersequent and display calculi-a unified perspective. Studia Logica, 102(6):1245-1294, 2014.

[23] Kosta Dosen and Peter Schroeder-Heister. Substructural Logics. Oxford University Press, 1994.

[24] J Michael Dunn. Gaggle theory: an abstraction of galois connections and residuation, with applications to negation, implication, and various logical operators. In Logics in AI, pages 31-51. Springer, 1991.

[25] J. Michael Dunn and Greg Restall. Relevance logic. Handbook of philosophical logic, 6:1-128, 2002.

[26] Raul Fervari. Relation-Changing Modal Logics. PhD thesis, Universidad Nacional de Córdoba, 2014.

[27] Tim French, Wiebe van der Hoek, Petar Iliev, and Barteld Kooi. On the succinctness of some modal logics. Artificial Intelligence, 197(0):56 - 85, 2013.

[28] Dov M Gabbay, Christopher John Hogger, John Alan Robinson, J Siekmann, and Donald Nute, editors. Handbook of logic in artificial intelligence and logic programming, volume Nonmonotonic reasoning and uncertain reasoning (Volume 3). Clarendon Press, 1998.

[29] Peter Gärdenfors. Knowledge in Flux (Modeling the Dynamics of Epistemic States). Bradford/MIT Press, Cambridge, Massachusetts, 1988.

[30] Peter Gärdenfors. Belief revision and nonmonotonic logic: Two sides of the same coin? In Logics in AI, pages 52-54. Springer, 1991.

[31] Gerhard Gentzen. Untersuchungen über das logische schließen. i. Mathematische zeitschrift, 39(1):176-210, 1935. 
[32] Laura Giordano, Valentina Gliozzi, Nicola Olivetti, Gian Luca Pozzato, and Camilla B Schwind. Non-classical logics for knowledge representation and reasoning. Intelligenza Artificiale, 5(1):127-131, 2011.

[33] Laura Giordano and Camilla Schwind. Conditional logic of actions and causation. Artificial Intelligence, 157(1):239-279, 2004.

[34] Jean-Yves Girard. Linear logic. Theoretical computer science, 50(1):1-101, 1987.

[35] Patrick Girard, Jeremy Seligman, and Fenrong Liu. General dynamic dynamic logic. In Thomas Bolander, Torben Braüner, Silvio Ghilardi, and Lawrence S. Moss, editors, Advances in Modal Logic, pages 239-260. College Publications, 2012.

[36] Rajeev Goré. Substructural logics on display. Logic Journal of IGPL, 6(3):451-504, 1998.

[37] Rajeev Goré. Dual intuitionistic logic revisited. In Roy Dyckhoff, editor, Automated Reasoning with Analytic Tableaux and Related Methods, volume 1847 of Lecture Notes in Computer Science, pages 252-267. Springer Berlin Heidelberg, 2000.

[38] VN Grishin. On a generalization of the ajdukiewicz-lambek system. In A. Mikhailov, editor, Studies in Nonclassical Logics and Formal Systems, pages 315-334. Nauka, 1983.

[39] Andrzej Grzegorczyk. A philosophically plausible formal interpretation of intuitionistic logic. Journal of Symbolic Logic, 36(2):329-329, 1971.

[40] Barteld Kooi and Bryan Renne. Arrow update logic. The Review of Symbolic Logic, 4(04):536-559, 2011.

[41] Saul A. Kripke. Semantical analysis of intuitionistic logic i. In J.N. Crossley and M.A.E. Dummett, editors, Formal Systems and Recursive Functions, volume 40 of Studies in Logic and the Foundations of Mathematics, pages 92 - 130. Elsevier, 1965.

[42] Natasha Kurtonina. Frames and Labels. A Modal Analysis of Categorial Deduction. $\mathrm{PhD}$ thesis, Ph. D. Thesis, Onderzoeksinstituut voor Taal en Spraak, University of Utrecht \& Institute for Logic, Language and Computation, University of Amsterdam, 1995.

[43] Joachim Lambek. The mathematics of sentence structure. American mathematical monthly, 65:154-170, 1958.

[44] Joachim Lambek. On the calculus of syntactic types. In R. Jakobson, editor, Structure of language and its mathematical aspects, pages 166-178. American Mathematical Society, Providence, 1961.

[45] David Makinson. Bridges from classical to nonmonotonic logic. King's College, 2005.

[46] David Makinson and Peter Gärdenfors. Relations between the logic of theory change and nonmonotonic logic. In André Fuhrmann and Michael Morreau, editors, The Logic of Theory Change, volume 465 of Lecture Notes in Computer Science, pages 185-205. Springer, 1989. 
[47] Edwin D Mares. Relevant logic and the theory of information. Synthese, 109(3):345-360, 1996.

[48] Edwin D. Mares and Robert K. Meyer. Relevant logics. In Lou Goble, editor, The Blackwell guide to philosophical logic. Wiley-Blackwell, 2001.

[49] Maricarmen Martinez and Sebastian Sequoiah-Grayson. Logic and information. In Edward N. Zalta, editor, The Stanford Encyclopedia of Philosophy. CSLI publications Stanford, summer 2014 edition, 2014.

[50] Elliott Mendelson. Introduction to mathematical logic. CRC press, 1997.

[51] Michael Moortgat. Symmetries in natural language syntax and semantics: the lambekgrishin calculus. In Logic, Language, Information and Computation, pages 264-284. Springer, 2007.

[52] Reinhard Muskens, Johan van Benthem, and Albert Visser. Dynamics. In J van Benthem and A ter Meulen, editors, Handbook of logic and language, pages 607-670. Elsevier, 2011.

[53] Donald Nute and Charles B Cross. Conditional logic. In Dov Gabbay and F. Guenthner, editors, Handbook of philosophical logic, volume 4, pages 1-98. Kluwer Academic Pub, 2001.

[54] Hiroakira Ono. Proof-theoretic methods in nonclassical logic -an introduction. In Masako Takahashi, Mitsuhiro Okada, and Mariangiola Dezani-Ciancaglini, editors, Theories of Types and Proofs, volume Volume 2 of MSJ Memoirs, pages 207-254. The Mathematical Society of Japan, Tokyo, Japan, 1998.

[55] John Perry and David Israel. What is information? Information, Language, and Cognition, $1,1990$.

[56] Francesca Poggiolesi. Gentzen calculi for modal propositional logic, volume 32. Springer, 2010.

[57] Francesca Poggiolesi and Greg Restall. Interpreting and applying proof theories for modal logic. In New Waves in Philosophical Logic. Palgrave Macmillan, 2012.

[58] Frank P. Ramsey. General propositions and causality. In H.A. Mellor, editor, Philosophical Papers. Cambridge University Press, Cambridge, 1929.

[59] Cecylia Rauszer. An algebraic and Kripke-style approach to a certain extension of intuitionistic logic. Dissertationes Mathematicae, CLXVII, 1980.

[60] Greg Restall. Information flow and relevant logics. In Logic, Language and Computation: The 1994 Moraga Proceedings. CSLI, pages 463-477. csli Publications, 1996.

[61] Greg Restall. An Introduction to Substructural Logics. Routledge, 2000. 
[62] Greg Restall. Relevant and substructural logics. Handbook of the History of Logic, 7:289398, 2006.

[63] Richard Routley and Robert K Meyer. The semantics of entailment-ii. Journal of Philosophical Logic, 1(1):53-73, 1972.

[64] Richard Routley and Robert K Meyer. The semantics of entailment-iii. Journal of philosophical logic, 1(2):192-208, 1972.

[65] Richard Routley and Robert K Meyer. The semantics of entailment. Studies in Logic and the Foundations of Mathematics, 68:199-243, 1973.

[66] Richard Routley, Val Plumwood, and Robert K Meyer. Relevant logics and their rivals. Ridgeview Publishing Company, 1982.

[67] Alasdair Urquhart. Completeness of weak implication. Theoria, 37(3):274-282, 1971.

[68] Alasdair Urquhart. A general theory of implication. Journal of Symbolic Logic, 37(443):270, 1972.

[69] Alasdair Urquhart. Semantics for relevant logics. Journal of Symbolic Logic, pages 159169, 1972.

[70] Johan van Benthem. Exploring logical dynamics. CSLI publications Stanford, 1996.

[71] Johan van Benthem. Inference in action. Publications de l'Institut Mathématique-Nouvelle Série, 82(96):3-16, 2007.

[72] Johan van Benthem. The information in intuitionistic logic. Synthese, 167(2):251-270, 2009.

[73] Johan van Benthem. Logical Dynamics of Information and Interaction. Cambridge University Press, 2011.

[74] Hans van Ditmarsch, Wiebe van der Hoek, and Barteld Kooi. Dynamic Epistemic Logic, volume 337 of Synthese library. Springer, 2007.

[75] Hans P. van Ditmarsch, Wiebe van der Hoek, and Barteld P. Kooi. Public announcements and belief expansion. In Renate A. Schmidt, Ian Pratt-Hartmann, Mark Reynolds, and Heinrich Wansing, editors, Advances in Modal Logic, pages 335-346. King's College Publications, 2004.

[76] Heinrich Wansing. Sequent calculi for normal modal propositional logics. Journal of Logic and Computation, 4(2):125-142, 1994.

[77] Heinrich Wansing. Displaying Modal Logic. Kluwer Academic Publishers, Norwell, MA, USA, 1998.

[78] Heinrich Wansing. Sequent systems for modal logics. Handbook of philosophical logic, 8:61-145, 2002. 
[79] Heinrich Wansing. Constructive negation, implication, and co-implication. Journal of Applied Non-Classical Logics, 18(2-3):341-364, 2008.

[80] Heinrich Wansing. Falsification, natural deduction and bi-intuitionistic logic. Journal of Logic and Computation, 2013. 